\title{
ON THE DESIGN OF A BIODEGRADABLE POC-HA POLYMERIC CARDIOVASCULAR STENT
}

\author{
by \\ JOONAS ILMARI PONKALA \\ Presented to the Faculty of the Graduate School of \\ The University of Texas at Arlington in Partial Fulfillment \\ of the Requirements \\ for the Degree of \\ MASTER OF SCIENCE IN MECHANICAL ENGINEERING
}

THE UNIVERSITY OF TEXAS AT ARLINGTON

August 2009 
Copyright ( ) by Joonas IImari Ponkala 2009

All Rights Reserved 


\section{ACKNOWLEDGEMENTS}

I wish to acknowledge my appreciation to those who have played an important role in the successful completion of my thesis. I express my gratitude to my advisor Dr. Panayiotis Shiakolas for all of his help and support, and for providing me with an interesting research topic. I enjoyed working on a project where I was able to apply my knowledge to an application that has the ability to make an impact on quality of life and benefit society.

I also wish to express gratitude my committee members Drs. Jian Yang and Pranesh Aswath and the cardiologists, Drs. Emmanouil Brilakis and Subhash Banerjee of UTSW, for their suggestions and support during the project. Part of this work was performed under a grant from the UTA/UTSW Collaborative Research Program whose financial support for the initial phase of this research is acknowledged. I thank my fellow students Richard Tran, Tré Welch, Mohsin Rizwan, and Nikhil Kavadia for their assistance during this project.

My deepest gratitude goes to my family for their love and support in all of my academic endeavors.

July 20, 2009 


\section{ABSTRACT \\ ON THE DESIGN OF A BIODEGRADABLE POC-HA POLYMERIC CARDIOVASCULAR STENT}

Joonas Ilmari Ponkala, M.S.

The University of Texas at Arlington, 2009

Supervising Professor: Panayiotis S. Shiakolas

The current state of the art in coronary stent technology is mainly populated by metallic stents usually coated with certain drugs to increase biocompatibility, even though experimental biodegradable stents have appeared in the horizon. To design stents from biodegradable polymers one must accurately characterize their mechanical behavior for analysis and optimization.

This manuscript presents the process for evaluating the material properties for biodegradable biocompatible POC-HA polymeric composite, techniques for developing the stent models for finite element analysis, and development of material models for finite element analysis. The developed material models were utilized in finite element analysis to evaluate the suitability of the POC-HA material for coronary stent application. In addition, the advantages of using femtosecond laser machining to fabricate the POC-HA stent will be discussed. The methodology presented can be applied in the development of a biocompatible and biodegradable polymeric stent. 


\section{TABLE OF CONTENTS}

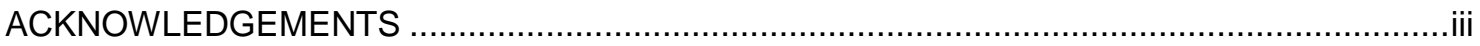

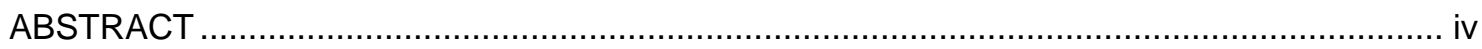

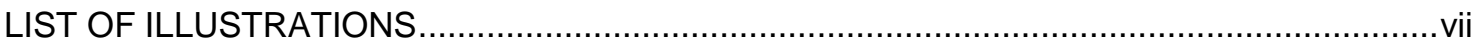

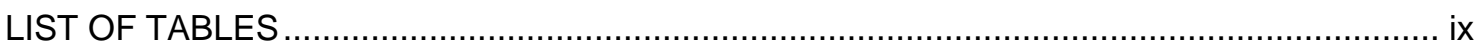

$\begin{array}{lll}\text { Chapter } & \text { Page }\end{array}$

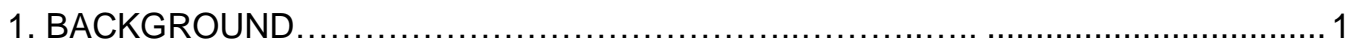

1.1 Cardiovascular Stents .................................................................. 1

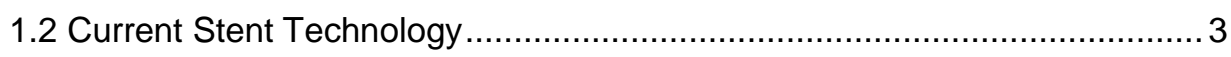

1.2.1 Bare Metal Stents ........................................................... 3

1.2.2 Drug Eluting Stents ........................................................ 4

1.2.3 Biodegradable Stents .................................................. 4

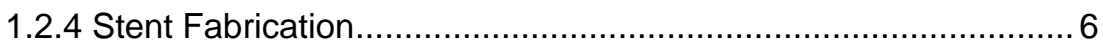

1.3 Requirements for a Biodegradable Coronary Stent .............................. 6

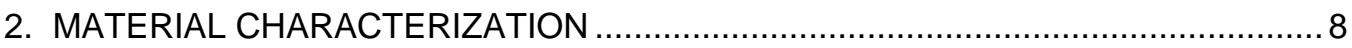

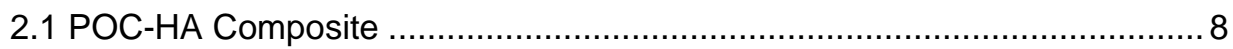

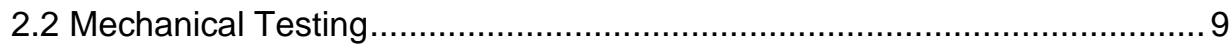

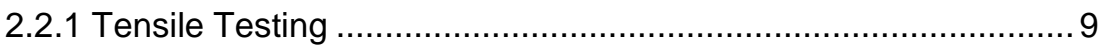

2.2.2 Compression Testing ................................................... 17

2.2.3 Cyclic Compression Testing ……………......................... 21

2.2.4 Wet Compression Testing............................................... 23

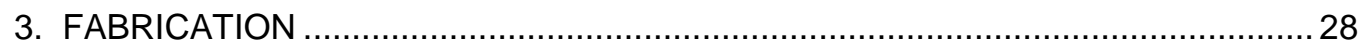

3.1 Femtosecond Laser Fabrication .................................................... 28

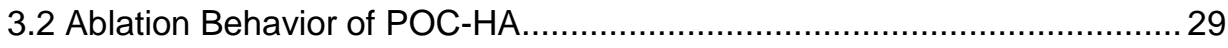




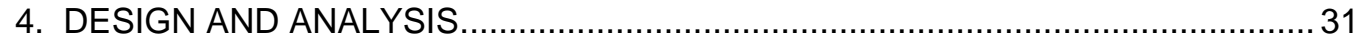

4.1 Finite Element Analysis Techniques ................................................ 31

4.2 Verification of Material Model ............................................................ 33

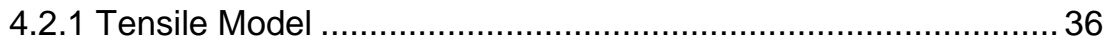

4.2.2 Compression Model....................................................... 41

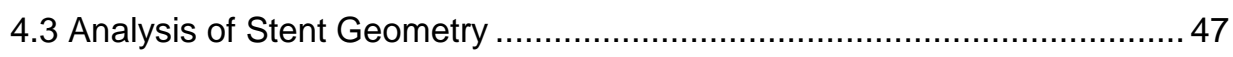

5. CONCLUSIONS AND RECOMMENDATIONS .............................................. 51

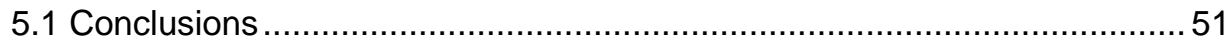

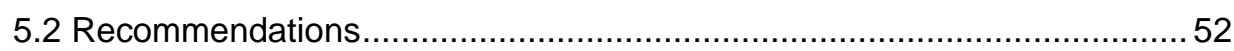

APPENDIX

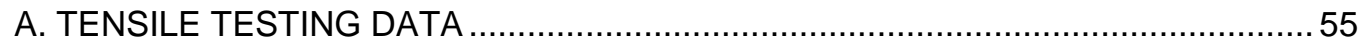

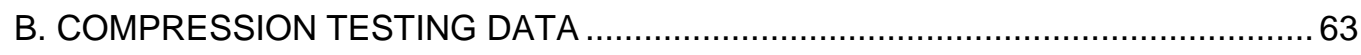

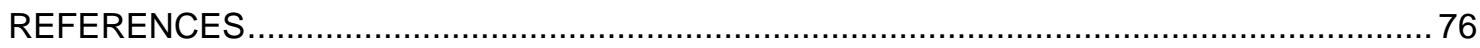

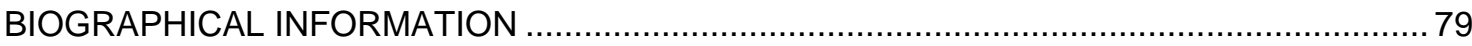




\section{LIST OF ILLUSTRATIONS}

$\begin{array}{lll}\text { Figure } & \text { Page }\end{array}$

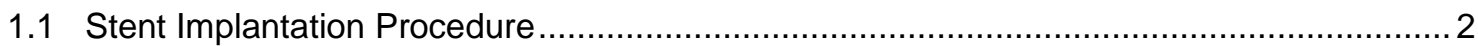

1.2 Vessel Healing Response following Bare-Metal Stent Implantation .................................

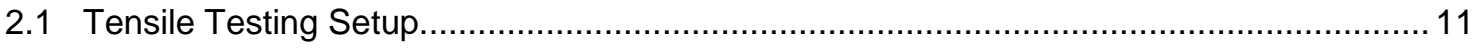

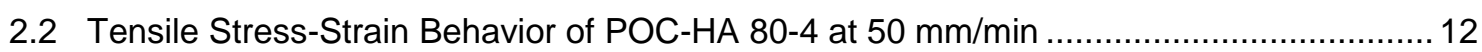

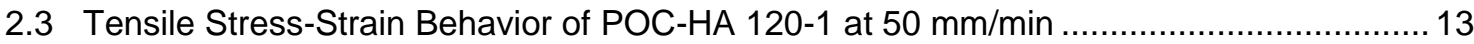

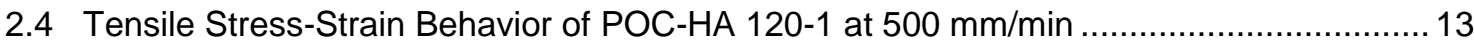

2.5 Overview of POC-HA Tensile Stress-Strain Behavior .............................................. 14

2.6 Failure Surface of a POC-HA 120-1 Tensile Specimen (scale bar 300 microns) ..............16

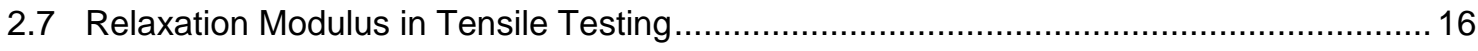

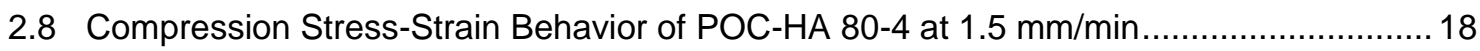

2.9 Compression Stress-Strain Behavior of POC-HA 120-1 at $1.5 \mathrm{~mm} / \mathrm{min} \ldots \ldots \ldots \ldots \ldots \ldots \ldots \ldots . . . . . . . . . . .18$

2.10 (a) Relaxation Modulus and (b) Normalized Relaxation Modulus for POC-HA 80-4 in Compression Testing ….............................................................. 20

2.11 (a) Relaxation Modulus and (b) Normalized Relaxation Modulus for POC-HA 120-1 in Compression Testing ..................................................................21

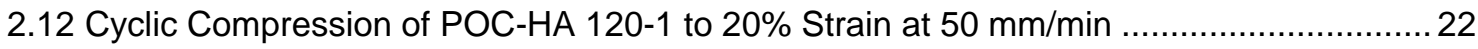

2.13 Stress-Strain Behavior of POC-HA 120-1 in Cyclic Compression ....................................22

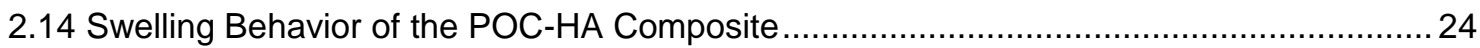

2.15 Comparison of POC-HA Stress-Strain Behavior for wet and dry Material Conditions ....... 25

2.16 Surface of a dry POC-HA 120-1 Compression Specimen

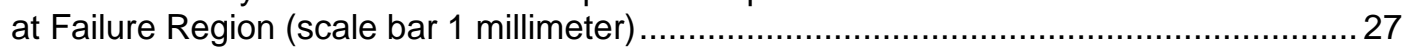

2.17 Surface of a POC-HA 120-1 Compression Specimen after wet Mechanical Testing (scale bar 1 millimeter).... 
3.1 Ablated Surface with 10 Pulses at $220 \mathrm{~mW}$ (scale bar 50 microns) ...............................24

3.2 Ablated Surface with 100 Pulses at $220 \mathrm{~mW}$ (scale bar 50 microns) ..............................24

4.1 Stent Finite Element Model Creation Steps:

(a) Strut Pattern Generated in Pro/ENGINEER,

(b) Generated Mesh with Element Definitions, Material Properties,

Boundary Conditions and Loading Specified in HyperMesh,

(c) Imported Finite Element Model in ANSYS, and

(d) Displacement Solution in ANSYS

4.2 ANSYS Prony Series Fit for POC-HA 120-1 Stress Relaxation in Compression............... 35

4.3 Tensile Verification Finite Element Model .................................................................. 36

4.4 Comparison of POC-HA 80-4 Material Models with Experimental Tensile Data ...............37

4.5 Comparison of POC-HA 120-1 Material Models with Experimental Tensile Data ............. 38

4.6 Comparison of Stress Relaxation Models to Experimental Tensile Data ..........................39

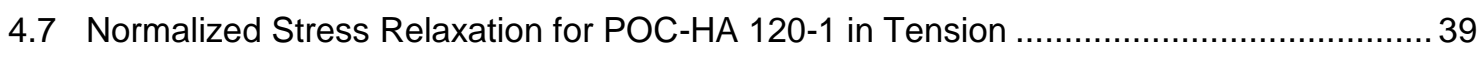

4.8 Compression Verification Finite Element Model ...................................................... 42

4.9 Comparison of POC-HA 80-4 Material Models to Experimental Compression Data ......... 42

4.10 Comparison of POC-HA 120-1 Material Models to Experimental Compression Data ....... 43

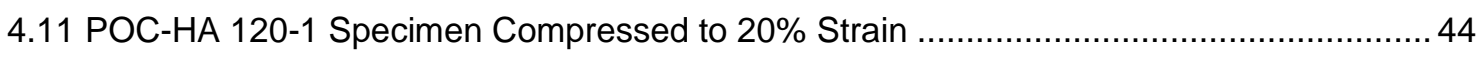

4.12 Half Width Lateral Displacements from the POC-HA 120-1

(a) Fixed End and (b) Free End Compression Verification Model .....................................4 44

4.13 Comparison of Stress Relaxation Models with Experimental Compression Data............. 45

4.14 The Normalized Stress Relaxation for POC-HA 120-1 in Compression ........................... 45

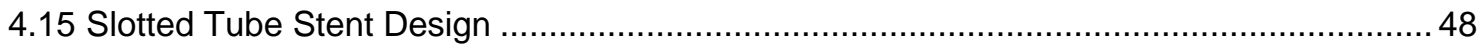

4.16 Quarter Strut Model with Equal Pressure Loading .................................................... 49 


\section{LIST OF TABLES}

Table

Page

2.1 Summary of POC-HA Mechanical Properties in Tension 15

2.2 Summary of POC-HA Mechanical Properties in Compression ......................................... 19

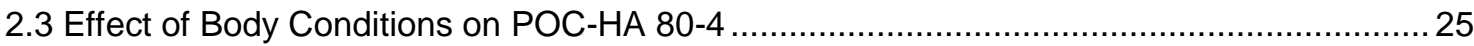

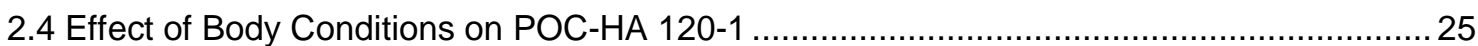

4.1 Material Properties for POCHA 80-4 ANSYS Tensile Model (Metric Units)........................ 40

4.2 Material Properties for POCHA 120-1 ANSYS Tensile Model (Metric Units)...................... 41

4.3 Material Properties for POCHA 80-4 ANSYS Compression Model (Metric Units) ...............46

4.4 Material Properties for POCHA 120-1 ANSYS Compression Model (Metric Units) ............. 46

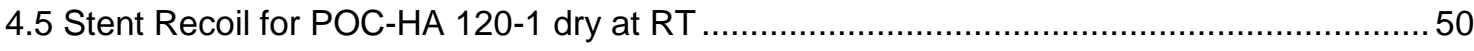

4.6 Stent Recoil for POC-HA 120-1 at Body Conditions......................................................50 


\section{CHAPTER 1}

\section{BACKGROUND}

Stents are tubular devices which are used to keep lumen open. Stents are utilized in interventional cardiology, urology, vascular applications, and to treat aortic aneyrusms. Stents can be made from metals or biodegradable materials in various geometric configurations tailored for the application.

\subsection{Cardiovascular Stents}

Coronary artery disease is caused by atherosclerosis which is accumulation of plaque on artery walls. The narrowing and hardening of arteries causes loss of blood flow leading to heart attack [Venkatraman, 2008].

In a process called angioplasty a balloon catheter with a stent is delivered to the blockage site and expanded to restore blood flow. The angioplasty process is shown in detail in Figure 1.1. The expanded stent supports the artery wall from collapsing thus restoring blood flow. A stent is a foreign material which causes a natural thrombogenic reaction in the body triggering blood platelet adhesion and coagulation [Kukreja, 2008]. The stent thrombosis can be prevented using dual antiplatelet therapy until endothelial cells encapsulate the stent and the thrombogenic struts are no longer in contact with blood [Kukreja, 2008]. The timeline of the healing process is shown in Figure 1.2.

The balloon expansion process and the stent cause damage to the artery wall resulting in neointimal hyperplasia, an exaggerated healing response [Kukreja, 2008]. The neointimal hyperplasia causes restenosis which is defined as reduction of lumen size [Brown, 2009].

Remodeling of the artery wall occurs due to restored blood flow and patient lifestyle. When metallic stents are used restenosis occurs until about six (6) months after implantation 
and the lumen size increases between (6) months and three (3) years after implantation [Kimura, 1996]. Therefore, the stent is no longer needed to provide scaffolding six (6) months after the intervention [Tamai, 2000].

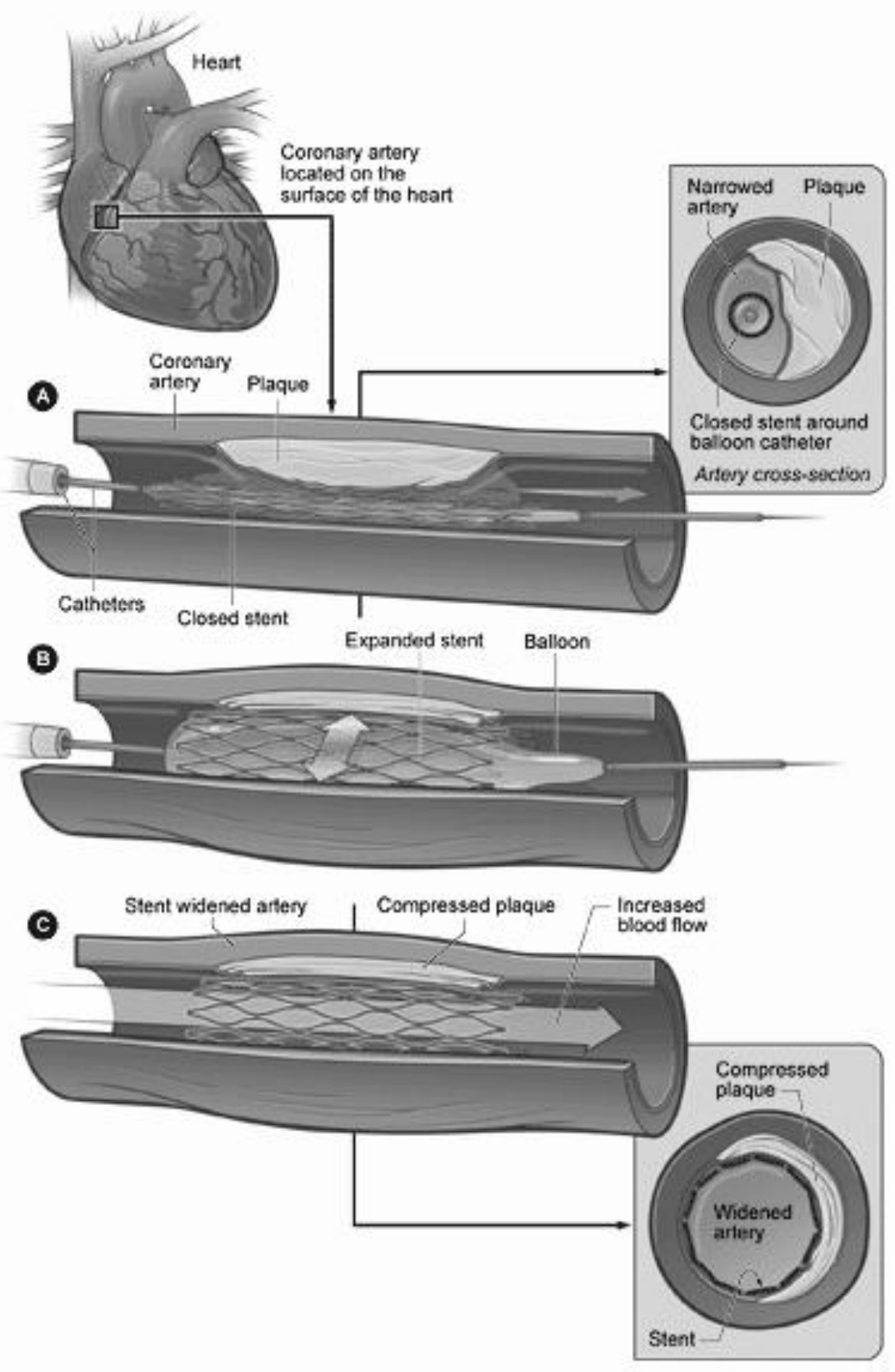

Figure 1.1 Stent Implantation Procedure [National Heart Lung and Blood Institute] 


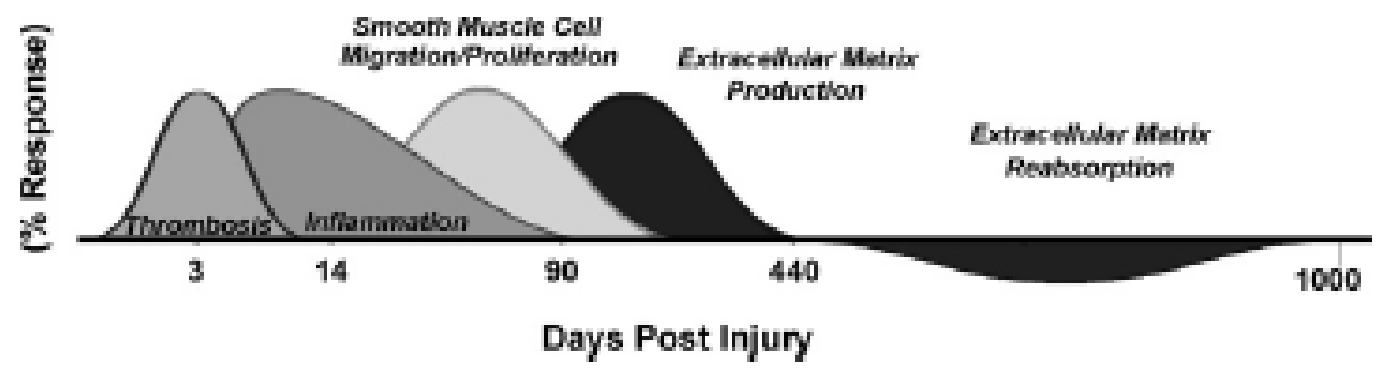

Figure 1.2 Vessel Healing Response following Bare-Metal Stent Implantation [Kukreja, 2008]

\subsection{Current Stent Technology}

\subsubsection{Bare Metal Stents}

Metallic stents have been used in interventional cardiology since their approval in 1994. Materials currently used include stainless steel, cobalt-chromium, and nitinol [Venkatraman, 2008]. With metallic stents the rate of restenosis was reduced to $20-25 \%$ from $30-40 \%$ with balloon angioplasty alone [Kukreja, 2008]. The problems associated with metallic stent technology are well documented in the literature. Since the stent will be in the body for the remainder of the life of the patient there are multiple possible complications associated with this technology. While the metallic stents can support high pressures they cause stent thrombosis and restenosis due to neointimal hyperplasia [Kukreja, 2008].

Another issue with metallic stents is their relatively high stiffness. In order to fabricate metallic stents with or close to blood vessel compliance characteristics, the dimensions of the stent struts can be small enough to act as blades when in contact with the artery wall [Morton, 2004]. The small dimensions result in stress concentrations at the artery wall which can cause inflammation. There is current research to evaluate the effect of the strut geometries on artery wall stresses using finite element methods [Capelli, 2009].

Metallic stents are usually delivered by balloon expansion. The stent is fabricated in the contracted or final shape, contracted into a catheter and delivered to the desired location. The stent is then expanded to the open state with an inflatable balloon catheter past the yielding 
point of the material resulting in a permanent final shape [Stoeckel, 2002]. The other delivery method is based on a self-expanding stent design in which the stent is fabricated in the final shape and then contracted for delivery in a catheter. Once delivered the stent will have enough elasticity to return to its original shape and keep the artery open. Nitinol stents utilize their shape memory properties for the delivery [Stoeckel, 2002]. In metallic stents the self-expanding stents cause more restenosis than balloon-expandable stents since the self-expanding design exerts outward pressure that stimulates greater growth of neointima [Morton, 2004].

\subsubsection{Drug Eluting Stents}

Currently metallic stents are coated with polymers that contain drugs to reduce neointimal hyperplasia. The current technology reduces restenosis by up to $60-80 \%$ as compared to bare metallic stents [Kukreja, 2008]. The problem that has emerged with the current drug eluting stents is late stent thrombosis which is attributed to incomplete endothelialization of the stent struts [Nakazawa, 2009]. There is also evidence that drug eluting stents may accelerate atherosclerosis [Nakazawa, 2009].

\subsubsection{Biodegradable Stents}

Biodegradable stents could solve the problem of in-stent restenosis associated with permanent stents and have the ability to deliver more drugs than thin stent coatings [Brown, 2009]. With fully absorbable stents there would be no need for long-term antiplatelet therapy and future surgical revascularization procedures would be possible [Kukreja, 2009].

The early attempts on biodegradable stents have been using poly(L-lactic acid) (PLLA) polymers. PLLA has good biocompatibility but it is brittle which makes the delivery of the stent problematic [Grabow, 2005]. Also PLLA is radiolucent so markers need to be incorporated into the design for visualization during the delivery and later diagnosis [Tamai, 2000]. The degradation rate of PLLA can be controlled but the inflammation response increases with lower molecular weight formulation [Venkatraman, 2008]. 
The first biodegradable coronary stent investigated in human clinical trials was the Igaki-Tamai PLLA stent introduced in 2000. The stent was delivered utilizing a heated balloon catheter which allows for self-expansion of the stent. The results indicated no stent thrombosis or stent recoil (reduction in stent diameter over time) with a restenosis rate of $10.5 \%$ [Tamai, 2000].

There are also drug eluting biodegradable stents. The BVS by Abbott Vascular is a PLLA stent coated with a poly(D,L-lactic acid) layer that contains everolimus, an antiproliferative agent. It has been evaluated in ABSORB clinical trials which show adequate scaffolding and no late term stent thrombosis one (1) year after implantation [Ormiston, 2008]. The stents were completely bioabsorbed two (2) years after implantation [Serruys, 2009]. An issue with the BVS stent is late stent recoil and strut malapposition (separation of stent struts from the artery wall) after 6 months [Tanimoto, 2008]. BVS stent shrunk after implantation while the Igaki-Tamai expanded over the same time frame. This behavior could be attributed to the delivery method. The balloon-expandable BVS stent recoils due to viscoelasticity to its constricted shape while the self expanding Igaki-Tamai stent expands to its unconstrained dimensions [Tanimoto, 2008].

Polymeric stents may require novel delivery methods. A PLLA fiber based coil-withincoil furled balloon-expandable stent has been developed by another group at The University of Texas at Arlington [Welch, 2008]. Biodegradable stents have also been developed from metals. A magnesium alloy stent has been shown to have less neointima formation over a $316 \mathrm{~L}$ stainless steel stent in animal experiments [Loos, 2007].

Other biodegradable polymeric materials used in stents include poly(lactic-co-glycolic acid) (PLGA), polyorthoester (POE), caprolactone (PCL), and chitosan - a natural polymer [Venkatraman, 2008]. An available biodegradable elastic polymer, poly(diol citrate) (POC) can be hybridized with bioabsorbable ceramic hydroxyapatite $(\mathrm{HA})$ in order to improve the mechanical properties and render the POC radiopaque. The POC-HA polymer has properties which render it suitable for stent application. The material is biocompatible and biodegradable 
with a controlled degradation rate. This ensures that once the stent is implanted, its mechanical behavior could be predicted through finite element modeling for accurate design for structural integrity as a function of degradation time. The degradation rate of POC-HA could be controlled from months to one (1) year [Qiu, 2006]. POC-HA is a radiopaque material which makes X-ray imaging of the complete stent during deployment and later for diagnostics and follow up possible as compared to the PLLA based stents. HA has been investigated as a coating material for stainless steel drug eluting stents to achieve more uniform drug release over time. In clinical trials the HA coated stent was effective in reducing lumen loss and neointimal hyperplasia at 4 and 9 months with no incomplete stent apposition [Costa, 2009].

\subsubsection{Stent Fabrication}

Fabrication techniques currently used for stents include laser cutting (Nd:YAG being most common), waterjet cutting, photo etching and wire forming [Stoeckel, 2002]. Femtosecond laser is a novel fabrication method that has not been yet utilized for fabrication of in-trial stents in the open literature.

\section{$\underline{1.3 \text { Requirements for a Biodegradable Coronary Stent }}$}

According to Venkatraman et. al. a biodegradable coronary stent must satisfy the following requirements:

a) Both the stent material and its degradation products must be biocompatible.

b) Structural integrity must be maintained for 6 months and full degradation should occur in about $12-18$ months.

c) The degradation of the stent must not result in pieces of degraded material being released into the lumen.

d) The stent must be deployable either by balloon expansion or by self-expansion.

e) The stent must be safely anchored after deployment and not migrate. It must also be able to structurally withstand the blood vessel contractions.

f) The stent must be radio-opaque to enable its safe and facile deployment. 
The main function of a stent is to provide scaffolding to keep the artery open. According to Venkatraman et. al. the stent must withstand minimum collapse strength of one (1) bar (comparable to a slotted-tube metal stent) [Venkatraman, 2008]. Collapse pressures for stents are characterized using pressure testing chambers where the stent is pressurized in a simulated artery (i.e. urethane tube) with increasing external pressure until it collapses [Lanzer, 2006].

When the stent is placed in the artery it is subject to compressive loads due to the plaque as well as the pulsations of the vessel during cardiac cycle. In metallic stents the acute recoil (diameter reduction immediately after implantation) is 4-6\% [Lanzer, 2006]. During the cardiac cycle the maximum diameter change of the human arteries has been measured to be $10 \%$ [Ozolanta, 1998]. Based on this information, the criteria for the stent to provide adequate scaffolding is diameter change of less than $10 \%$ for one (1) bar applied pressure. If the acute recoil of the stent exceeds $10 \%$ it can lose contact with the artery wall during cardiac cycle and start migrating with the blood flow.

These design requirements are translated into high stiffness for collapse pressure and scaffolding function but flexibility for delivery. Large stent strut dimensions are a problem for delivery as well as a source for complications in the blood vessels. If the stent struts are too thick, they could disrupt the blood flow in the vessel. For metallic stents, it has been observed that thicker struts also cause delayed endothelialization [Morton, 2004]. If the struts are too wide, i.e. cover too much of the surface area of the vessel, the endothelium will not properly cover the stent. Also, long stents have higher restenosis rates than short stents [Morton, 2004]. 


\section{CHAPTER 2}

\section{MATERIAL CHARACTERIZATION}

\subsection{POC-HA Composite}

The POC-HA biodegradable composite consist of poly(1,8-octanediol-co-citrate) (POC) and hydroxyapatite (HA). POC mechanical properties and degradation rates can be controlled with the synthesis conditions and its preparation does not involve any non-biocompatible solvents or catalysts. HA is a bioceramic which can be found naturally in bones and can be used to alter or tailor the mechanical properties of the composite. POC-HA materials have been used in hard tissue engineering applications such bone screw implants [Qiu, 2006].

POC was synthesized under a controlled polycondensation reaction between citric acid and 1,8 -octanediol at $140{ }^{\circ} \mathrm{C}$ to yield a viscous low molecular weight pre-polymer. Following the pre-polymer synthesis, HA was added to the pre-polymer to create a 1:1 polymer:ceramic ratio. This ratio was chosen based on previous work on the composite mechanical properties [Qiu, 2006]. The POC pre-polymer and the HA powder were mixed to form a clay like mass which was freeze dried in vacuum for one (1) day to remove the solvent. If the solvent is present in the post-polymerization steps it will evaporate causing porosity in the material. The dried mass can be then formed into desired shapes using an appropriate mold. These shapes were then postpolymerized at $80^{\circ} \mathrm{C}$ for four (4) days and additional $120^{\circ} \mathrm{C}$ for one (1) day to achieve the desired crosslinking characteristics.

For stent fabrication the POC-HA polymer was formed into tubular structures which have the desired final outer diameter and strut thickness of the stent. The tubes have been fabricated using dip-coating, motorized spinning, and hand rolling techniques onto glass rods. 
The two materials characterized in this study, POC-HA crosslinked at $80^{\circ} \mathrm{C}$ for 4 days and POC-HA crosslinked at $80{ }^{\circ} \mathrm{C}$ for four (4) days plus $120^{\circ} \mathrm{C}$ for one (1) additional day, will be referred as POC-HA 80-4 and POC-HA 120-1 respectively in the document.

\subsection{Mechanical Testing}

During its lifetime a vascular stent will experience a complex loading pattern. In delivery the stent will be subject to high strains because it must be fitted into a delivery device, usually a catheter, and navigated through the body to the correct location in the artery. When implanted the vascular stent must oppose the plaque and keep the artery from collapsing. With polymer materials the viscoelasticity will cause recoil of the stent after the delivery [Grabow, 2005]. Then the stent will be subject to cyclic loading due to the blood pressure change in the cardiac cycle. Therefore, the stress-strain behavior with appropriate material properties (Young's modulus, Poisson's ratio, and shear modulus), failure strain, stress relaxation, and hysteresis behavior of a stent material must be characterized. With biodegradable polymers the stent must also be able to have adequate strength as the material degrades over time until the plaque has been healed. The mechanical properties must be evaluated for dry at room temperature conditions for delivery purposes and wet at body temperature conditions for the loads experienced in the artery.

During the cardiac cycle arteries can experience a diameter change of up to $10 \%$ which occurs twice in every cycle [Ozolanta, 1998]. Assuming a heart rate of $1 \mathrm{~Hz}$ this results in strain rates which are beyond testing machine capabilities in our laboratory and probably too fast for the stent to react and respond to. Therefore material testing was performed at strain rates found in published standards and open literature and not at rates which are present in the actual artery.

\subsubsection{Tensile Testing}

Tensile testing for material property characterization was performed using uniform width strip specimens instead of dog bone shaped specimens. The strip specimens were chosen over 
dog bone shaped specimens because they do not require any special setup to measure the actual strains in the specimen and use less material. In the strip specimen, the elongation is uniform in the entire specimen and the crosshead displacement can be used directly to calculate the strain where with dog bone specimens one must use an extensometer to measure the actual length change in the uniform section or correct the acquired data to account for the non-uniform elongations of the specimen. The benefit of using a dog bone specimen as compared to a strip specimen is that one can obtain the actual failure point of the material. When strip specimens are used the failure is expected to occur at the grips (a stress concentration) and at strains below the actual failure point. Using data from preliminary experiments performed in our laboratory on the POC-HA material with strip and dog bone specimens it was determined that the actual failure point of the material is beyond the $10 \%$ strain range which is of interest in stent design. If the material behaves well in the strain region of interest, from a design point of view the actual failure strain or stress of the material will not be reached in the stent application and does not need to be evaluated.

The POC-HA specimens for tensile testing were prepared by crosslinking the composite in a mold to $110 \mathrm{~mm}$ long, $6 \mathrm{~mm}$ wide, and around $1 \mathrm{~mm}$ thick strips. The specimen dimensions were measured with vernier calipers with an accuracy of $0.01 \mathrm{~mm}$. The free length of the specimen was measured by first zeroing the crosshead with no separation between grips. After the specimen was placed in the grips the crosshead was moved to remove slack in the specimen by monitoring the force sensor of the tensile testing machine. At this point the crosshead position indicated the exact free length of the specimen.

The specimens were tested with an MTS Insight $2 \mathrm{kN}$ bench top mechanical testing system. The tensile testing setup with pneumatic grips is shown in Figure 2.1. The aspect ratio for the specimens was 10:1 (L:D) between grips with material covering the full length of the grip faces to ensure even gripping and no slipping. The strain was calculated from the crosshead position for all experiments. The grip pressures used in the experiments were 15 psi for POC- 
HA 80-4 and 20 psi for POC-HA 120-1. The POC-HA 120-1 material is a harder compound and is able to sustain higher forces exerted by the serrated grip faces without significant damage.

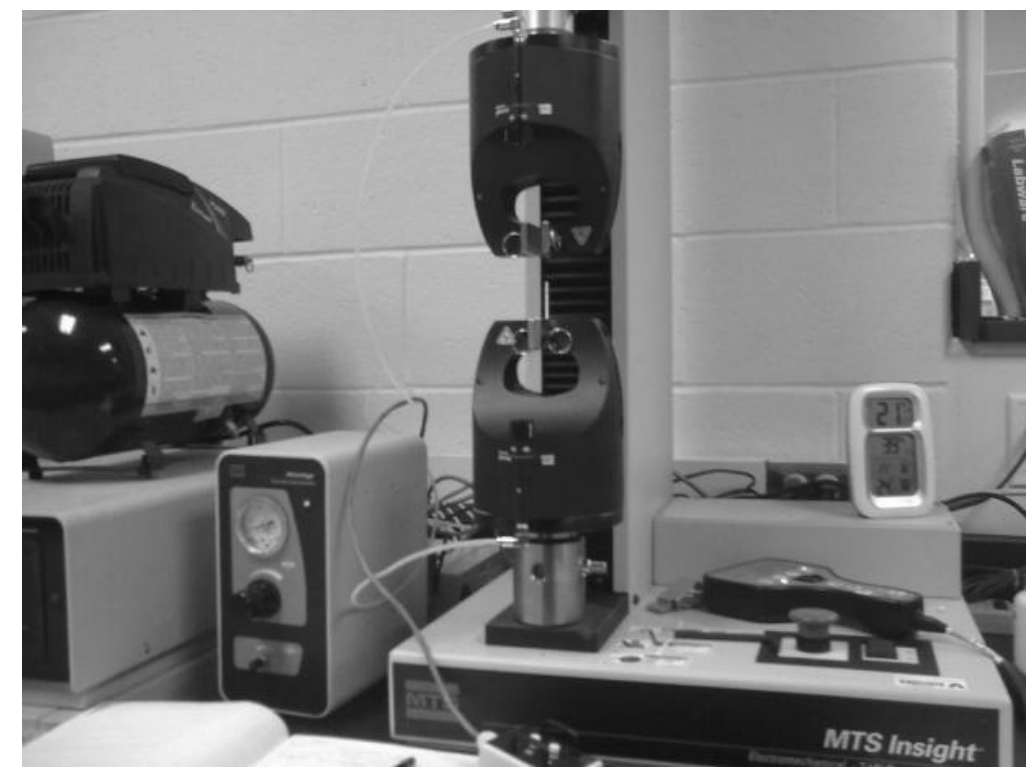

Figure 2.1 Tensile Testing Setup

The specimens were tested at two different crosshead speeds to determine the strain rate dependency of the material. The testing speed of $500 \mathrm{~mm} / \mathrm{min}$ was chosen based on the ASTM Standard D412. The other crosshead speed was selected at $50 \mathrm{~mm} / \mathrm{min}$. Both material conditions $(\mathrm{N}=3)$ were loaded until failure at $50 \mathrm{~mm} / \mathrm{min}$. For the POC-HA $120-1$ specimens $(\mathrm{N}=3)$ were also loaded to failure at $500 \mathrm{~mm} / \mathrm{min}$. The $\mathrm{DAQ}$ rate was selected at $50 \mathrm{~Hz}$. The stress-strain curves for these tests are presented in Figures 2.2, 2.3, and 2.4

The stress relaxation behavior was evaluated from data obtained when the specimens were loaded at $500 \mathrm{~mm} / \mathrm{min}$ to $10 \%$ strain and held for 1 hour. This test also provides the stress-strain behavior for the higher strain rate. The data was recorded at $100 \mathrm{~Hz}$ during loading and for the first minute of the relaxation to accurately capture the behavior in this region of most interest. For the next nine minutes, the DAQ rate was $10 \mathrm{~Hz}$ and for the remaining 50 minutes of 
relaxation the rate was $1 \mathrm{~Hz}$ since it is expected that there should not be significant changes in the force readings at this time interval.

A characteristic of the tensile testing was the high variation in the results. With the same testing conditions there was either a decaying nonlinear or a near linear behavior until failure for both materials. Figures 2.3 and 2.4 show the stress-strain behavior for the same POC-HA 120-1 material with different strain rates. Based on these results no conclusions could be drawn about the strain rate dependency due to the inconsistency in the data. The averaged stress values at specified strains are presented in Figure 2.5 with error bars representing standard deviations in the data. The high deviation is clearly present for POC-HA 120-1 material and the trend for the strain dependency contradicts theory. With a higher strain rate the material should behave stiffer since there is less time for the viscoelastic behavior to take effect [Brown, 2009].

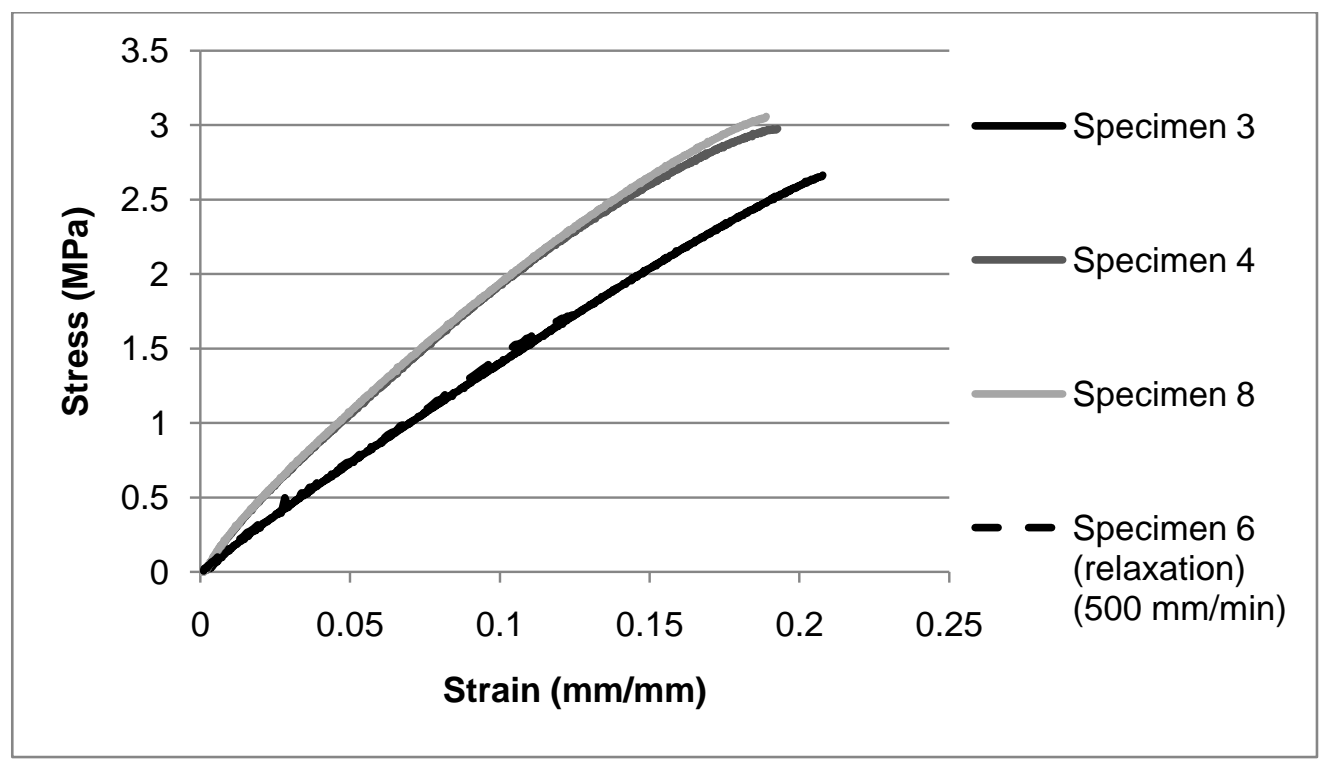

Figure 2.2 Tensile Stress-Strain Behavior of POC-HA 80-4 at $50 \mathrm{~mm} / \mathrm{min}$ 


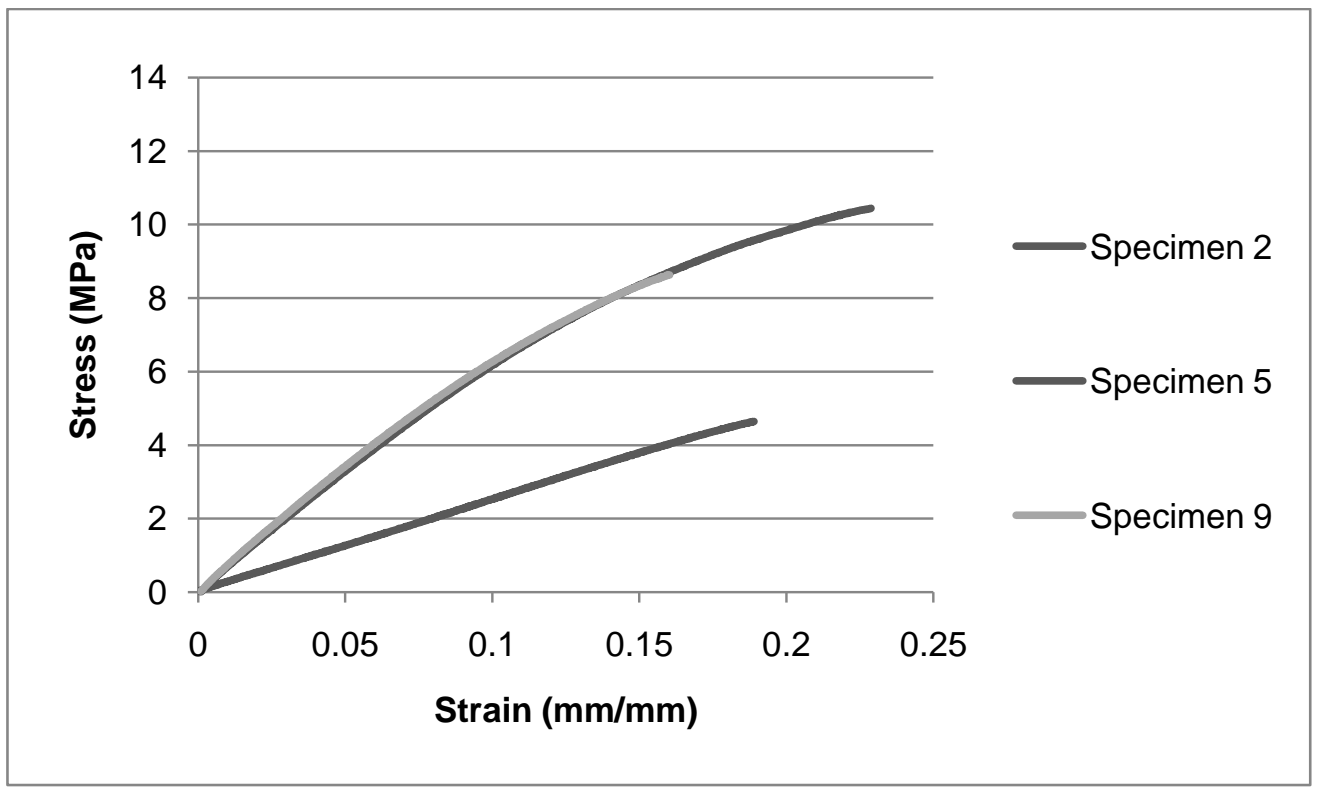

Figure 2.3 Tensile Stress-Strain Behavior of POC-HA 120-1 at $50 \mathrm{~mm} / \mathrm{min}$

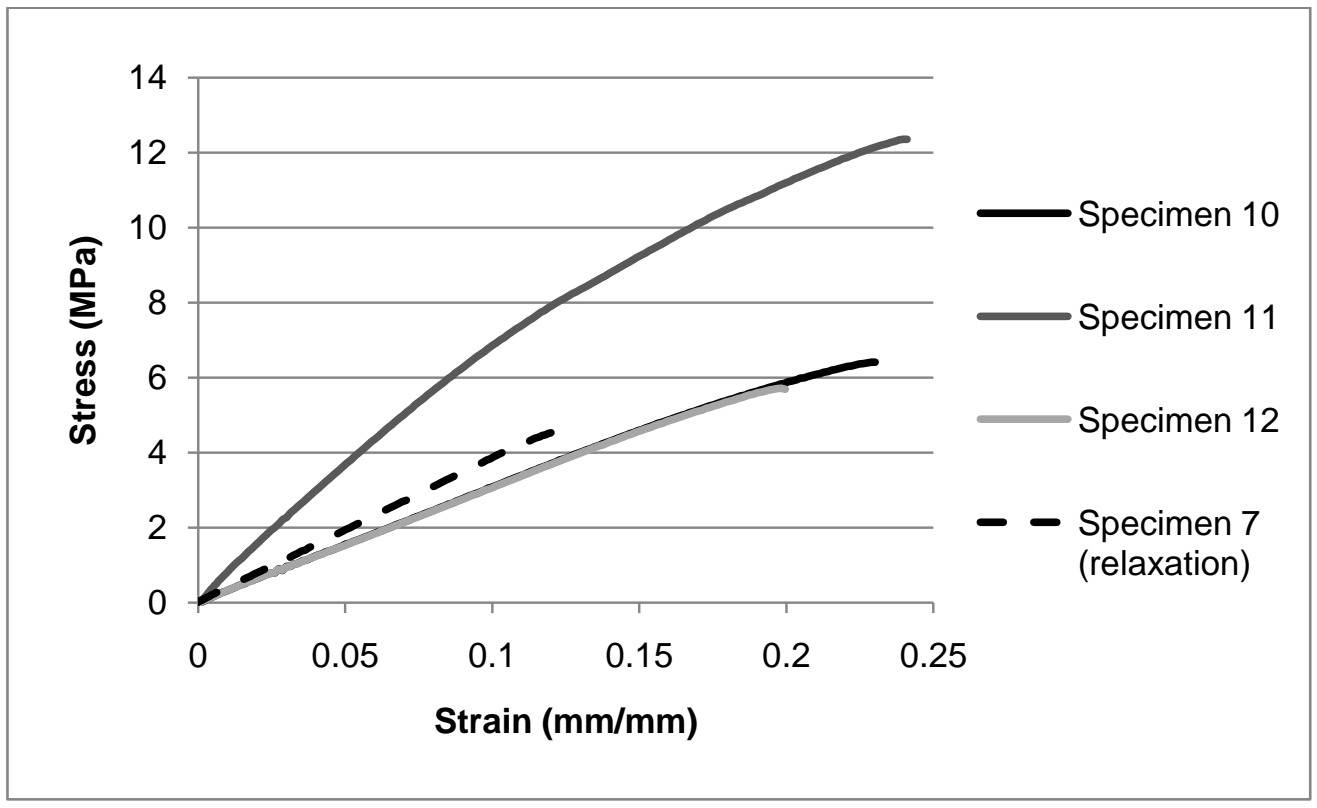

Figure 2.4 Tensile Stress-Strain Behavior of POC-HA 120-1 at $500 \mathrm{~mm} / \mathrm{min}$ 


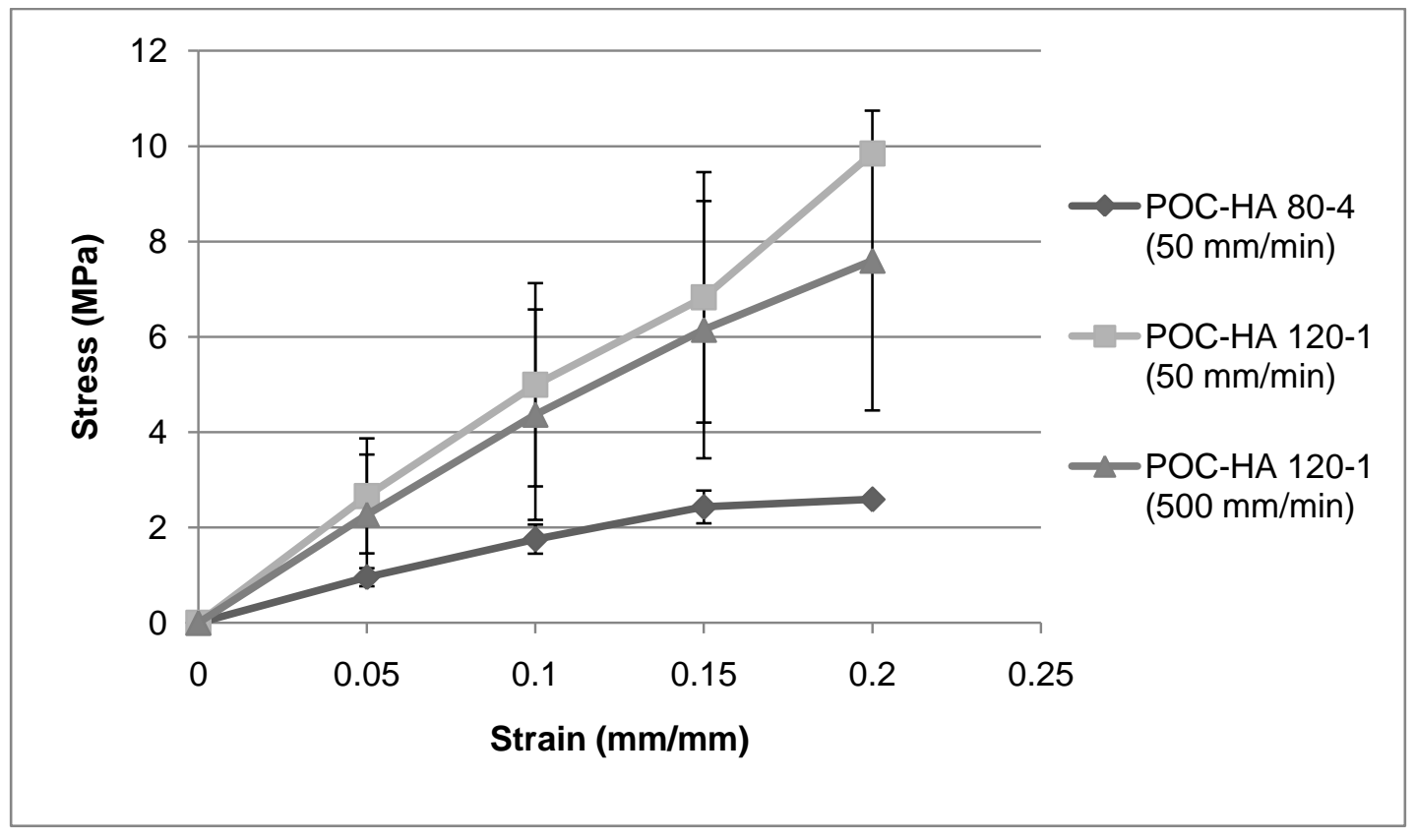

Figure 2.5 Overview of POC-HA Tensile Stress-Strain Behavior

For each of the specimens two regions of near linear behavior were identified. First the 0 to $10 \%$ strain region which is the main region of interest in stent design and second the 10 to $20 \%$ strain range. For each of these regions a least squares linear fit was applied to calculate the elastic Young's modulus of the material. The average of these results for estimating the material properties of POC-HA in tension are summarized in Table 2.1. The fitted stress-strain plots for each of the tensile experiments are included in Appendix A.

The tensile experiments were videotaped for further analysis. Still frames were captured from the video at initial and maximum strain to determine the dimension changes in the specimens. The Poisson's ratio was calculated to be 0.39 for POC-HA $80-4$ and 0.31 for POCHA 120-1 from measurements using ImageJ software [Rasband, 1997]. The videos were also utilized to observe the instanteneous recovery behavior of the material after load removal. It was observed that over $70 \%$ of the displacement was instantenously recovered for both materials. This instanteneous recovery is an indication of the elasticity or springiness of the Maxwell model for the material, i.e. parallel spring and dashpot elements. 
Table 2.1 Summary of POC-HA Mechanical Properties in Tension

\begin{tabular}{|c|c|c|c|c|c|c|c|c|c|}
\hline \multirow[b]{2}{*}{ Material } & \multicolumn{2}{|c|}{ Young's Modulus (MPa) } & \multicolumn{4}{|c|}{ Stress (MPa) at Strain (\%) } & \multirow{2}{*}{$\begin{array}{c}\text { Max } \\
\text { Stress } \\
\text { (MPa) }\end{array}$} & \multirow[b]{2}{*}{$\begin{array}{c}\text { Failure } \\
\text { strain }\end{array}$} & \multirow[b]{2}{*}{$\begin{array}{c}\text { Poisson's } \\
\text { Ratio }\end{array}$} \\
\hline & $\begin{array}{c}0-10 \% \\
\text { Strain }\end{array}$ & $\begin{array}{c}10-20 \% \\
\text { Strain }\end{array}$ & & $10 \%$ & $15 \%$ & $20 \%$ & & & \\
\hline 80-4 Dry RT & 17.21 & 12.09 & 0.96 & 1.76 & 2.43 & 2.59 & 2.90 & $20 \%$ & 0.39 \\
\hline 120-1 Dry RT & 1970 & & 2.66 & 4.99 & 6.83 & 9.85 & 7.88 & $19.26 \%$ & 0.31 \\
\hline$\%$ Change: & 189.22 & 176.08 & 178.11 & 184.38 & 180.78 & 279.8 & 171.85 & -1.93 & \\
\hline
\end{tabular}

According to the results presented in Table 2.1, the material with the increased crosslinking exhibits a significant improvement in stiffness and load bearing capacity and a slight drop in failure strain. The tensile modulus was determined to be $17.2 \mathrm{MPa}$ for POC-HA 80-4 and 49.8 MPa for POC-HA 120-1 in the strain range of 0 to $10 \%$. For comparison the tensile material properties for PLLA are 2.58 GPa Young's modulus, 44.9 MPa tensile strength, and $5.5 \%$ failure strain (elongation at break) [Grabow, 2005]. The results in Table 2.1 were used to develop the material model for finite element analysis.

An important observation from the tensile testing is the absence of a yielding region before failure. This is important for stent design and delivery since it eliminates the balloonexpansion method which utilizes the plastic flow of the material during delivery. The stent design for POC-HA should be restricted to self-expanding configurations. This determines that the stent pattern shall be fabricated in the open configuration and the pattern shall allow for crimping without material failure.

A failure surface of a POC-HA 120-1 tensile specimen is presented in Figure 2.6. The scanning electron microscopy (SEM) image revealed that there were voids present in the molded tensile specimens. The voids can be attributed to trapped air during the molding of the specimen. The size of the voids was in the range of 100 by 50 microns and the smaller dimension was in the direction of compression in the specimen mold. The failure surface was not smooth but had a flaky texture.

The stress relaxation for the tensile testing at $10 \%$ strain is presented in Figure 2.7. The relaxation modulus was calculated by dividing the stress value at a time point by the constant 
strain. This information can be used to define the viscoelastic behavior in finite element modeling. It was observed that the POC-HA 120-1 material exhibits a faster rate of stress relaxation.

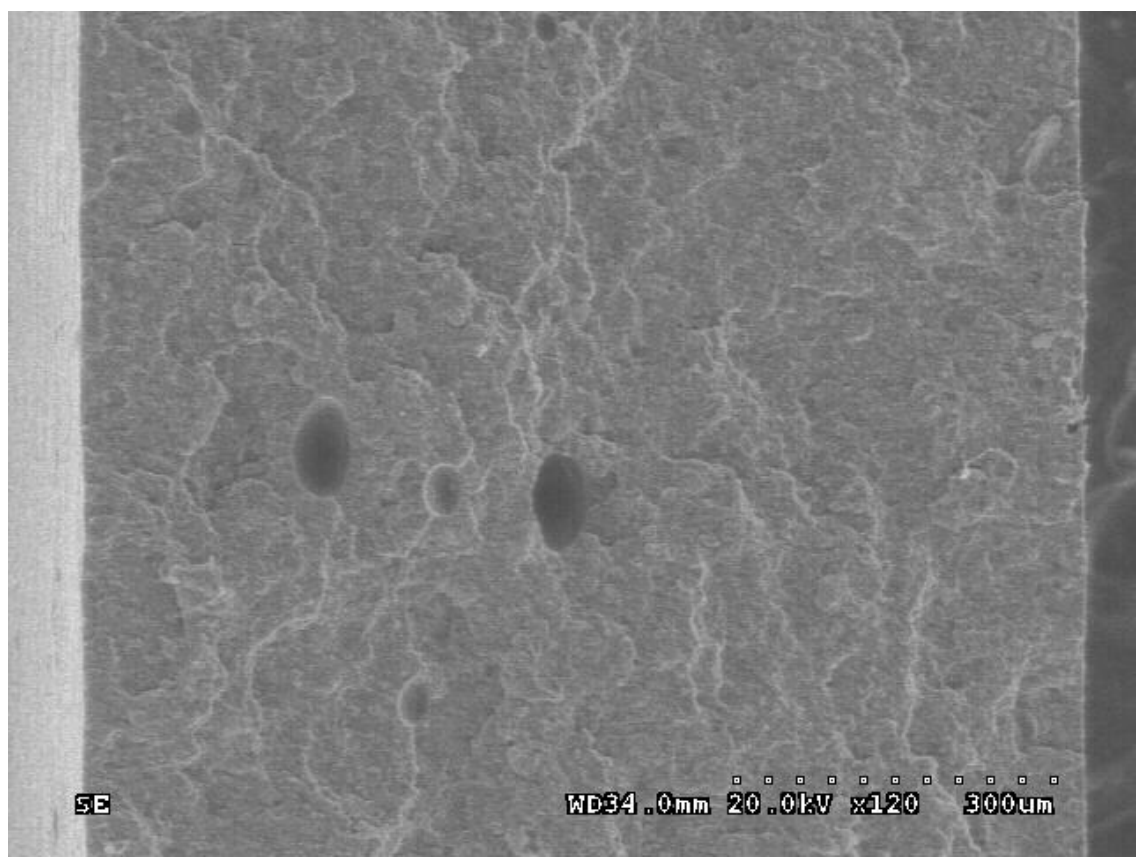

Figure 2.6 Failure Surface of a POC-HA 120-1 Tensile Specimen (scale bar 300 microns)

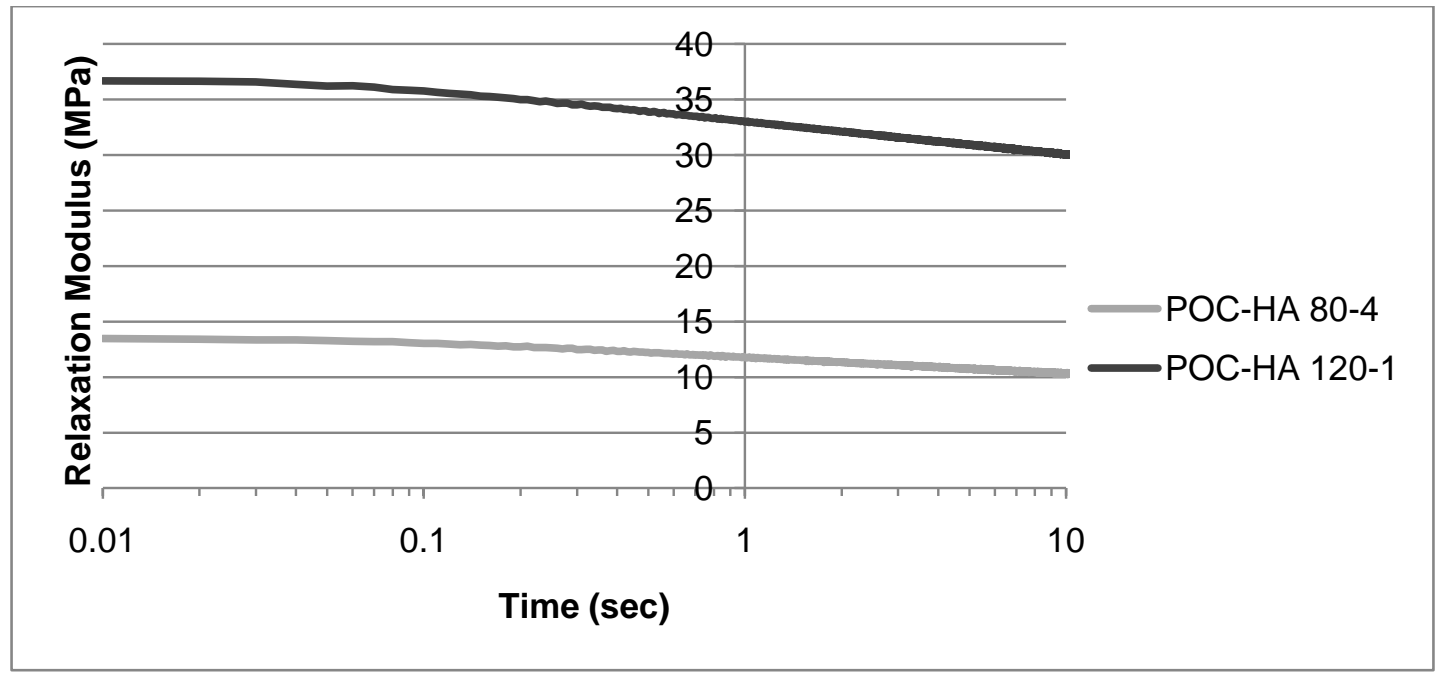

Figure 2.7 Relaxation Modulus in Tensile Testing 


\subsubsection{Compression Testing}

Cylindrical specimens were used in compression testing. In specimen fabrication, the POC-HA composite was first compacted in a metallic cylindrical mold with a piston to a diameter of $5 \mathrm{~mm}$ and 2:1 aspect ratio per ASTM Standard D695. The specimens were then removed from the metallic mold and placed in tubular Teflon molds with diameter of $4.5 \mathrm{~mm}$ for crosslinking. After crosslinking the specimen ends were cut square with a razor blade and dimensions were taken with vernier calipers with an accuracy of $0.01 \mathrm{~mm}$. The diameter of the specimen was measured at three locations and averaged. The specimens were tested with an MTS Insight $2 \mathrm{kN}$ bench top mechanical testing system with compression plates. The strain was calculated from the crosshead position for all experiments. The specimens were axially compressed to $50 \%$ strain with a crosshead speed of $1.5 \mathrm{~mm} / \mathrm{min}$ based on the ASTM Standard D695. To evaluate stress relaxation behavior, the specimens were loaded at a crosshead speed of $50 \mathrm{~mm} / \mathrm{min}$ to $20 \%$ strain and held for 1 hour.

The stress-strain plots for compression testing are presented in Figures 2.8 and 2.9. Overall the compressive behavior was more repeatable than the behavior in tensile testing. In compression the increased strain rate did not have a major effect on the material stiffness. Based on one specimen there was a slight increase of material stiffness for POC-HA 80-4. For POC-HA 120-1 one specimen followed the behavior of the slower strain rate while the other specimen behaved less stiff. Again no clear conclusions can be drawn on the strain rate effect. In previous research conducted in our laboratory on POC-HA material it was observed that there was no significant strain-rate effect in the material in shear testing.

In the POC-HA 80-4 specimens no failures were observed at $50 \%$ strain. In the POCHA 120-1 specimens the failures occurred near the ends of the specimen where the free face meets the compression plate and not at the center of the specimen. This is attributed to the high friction force at the compression plates which prevents the material at the interface from 
expanding radially. The compression tests were also videotaped to analyze the material expansion and behavior during testing.

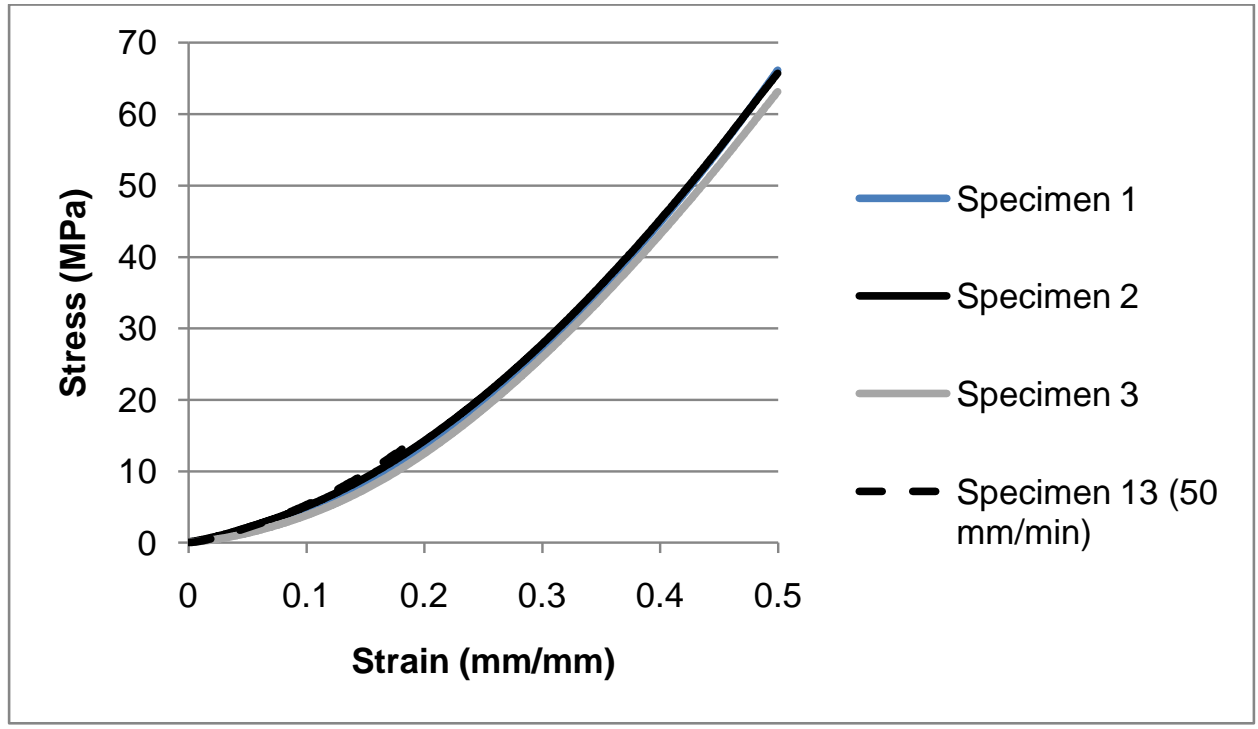

Figure 2.8 Compression Stress-Strain Behavior of POC-HA $80-4$ at $1.5 \mathrm{~mm} / \mathrm{min}$

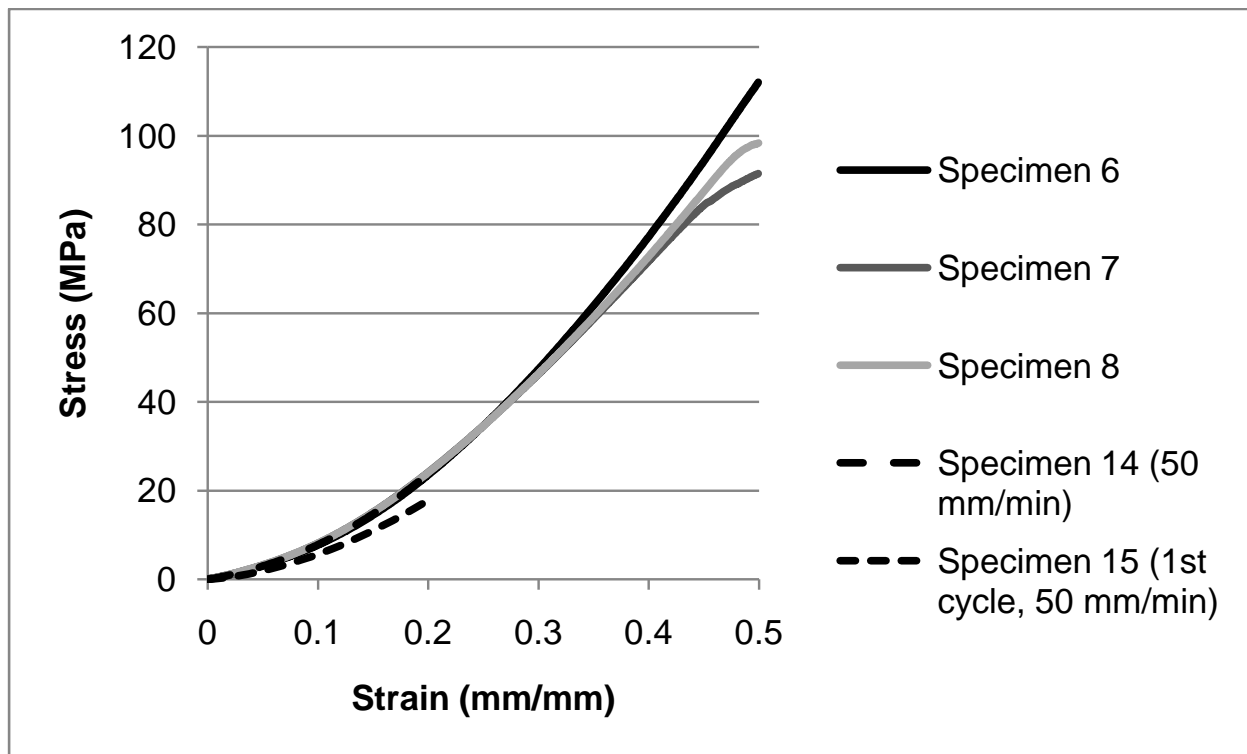

Figure 2.9 Compression Stress-Strain Behavior of POC-HA $120-1$ at $1.5 \mathrm{~mm} / \mathrm{min}$ 
For each of the specimens two regions of near linear behavior were identifed. First the 0 to $10 \%$ strain region which is the main region of interest in stent design and second the 10 to $20 \%$ strain range. For each of these regions a least squares linear fit was applied to calculate the elastic Young's modulus of the material. The average of these results for material characterization in compression are presented in Table 2.2. The fitted stress-strain plots for each of the tensile experiments are included in Appendix B.

Table 2.2 Summary of POC-HA Mechanical Properties in Compression

\begin{tabular}{|c|c|c|c|c|c|c|c|c|}
\hline \multirow[b]{2}{*}{ Material } & \multicolumn{2}{|c|}{ Young's Modulus (MPa) } & \multicolumn{5}{|c|}{ Stress $(\mathrm{MPa})$ at Strain (\%) } & \multirow[b]{2}{*}{$\begin{array}{l}\text { Max. } \\
\text { strain }\end{array}$} \\
\hline & $\begin{array}{l}0-10 \% \\
\text { Strain }\end{array}$ & $\begin{array}{l}10-20 \% \\
\text { Strain }\end{array}$ & $5 \%$ & & & & $30 \%$ & \\
\hline 80-4 Dry RT & 45.59 & 92.33 & 1.75 & 4.49 & 8.39 & 13.49 & 27.08 & $50 \%$ \\
\hline 120-1 Dry RT & 79.61 & 159.84 & 3.22 & 8.0 & 14.96 & 23.94 & 46.75 & $46 \%$ \\
\hline \% Change: & 74.64 & 73.11 & 84.00 & 79.14 & 78.19 & 77.52 & 72.65 & -7.33 \\
\hline
\end{tabular}

According to the results presented in Table 2.2, the material with the increased crosslinking exhibits a significant improvement in stiffness and load bearing capacity. The compressive Young's modulus was determined to be 45.6 MPa for POC-HA 80-4 and 79.6 MPa for POC-HA $120-1$ in the strain range of 0 to $10 \%$. The results in Table 2.2 were used to develop the material model for finite element analysis.

The stress relaxation behavior for compression testing at $20 \%$ strain for the two materials is presented in Figures 2.10 and 2.11. The relaxation modulus was calculated by dividing the stress value at a time point by the constant strain. The relaxation testing was performed three times for each specimen to investigate the effect of repeated loading. The recovery of the specimens was measured with calipers after compression. The retesting was performed after the specimens had recovered to their original outer dimensions which occurred after about three days. 


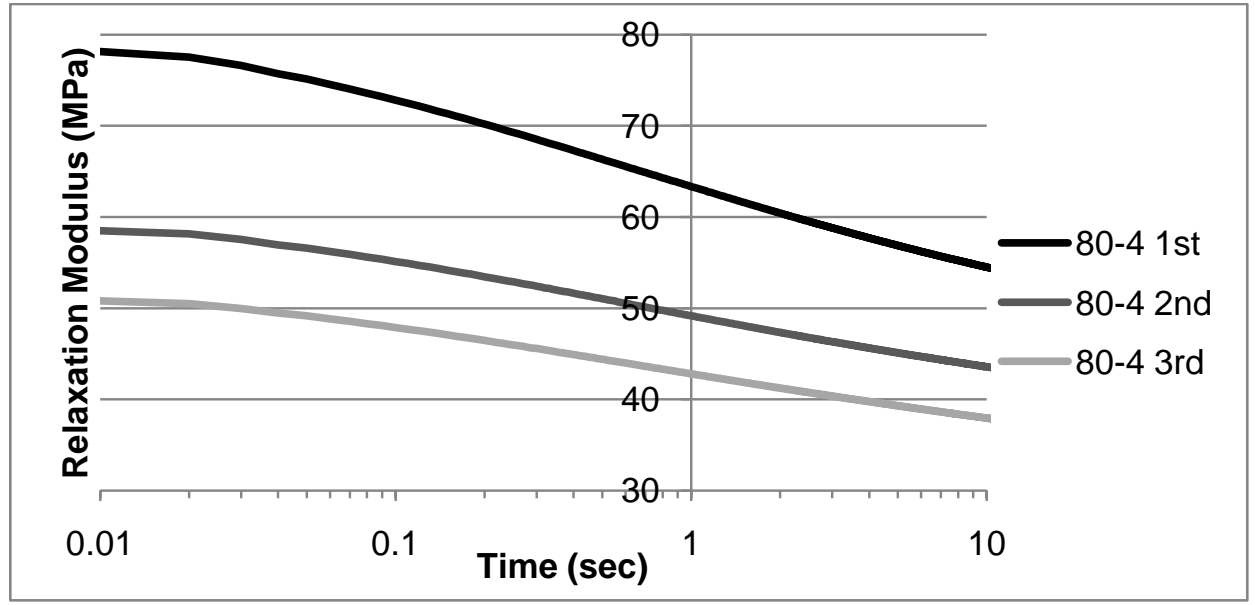

(a)

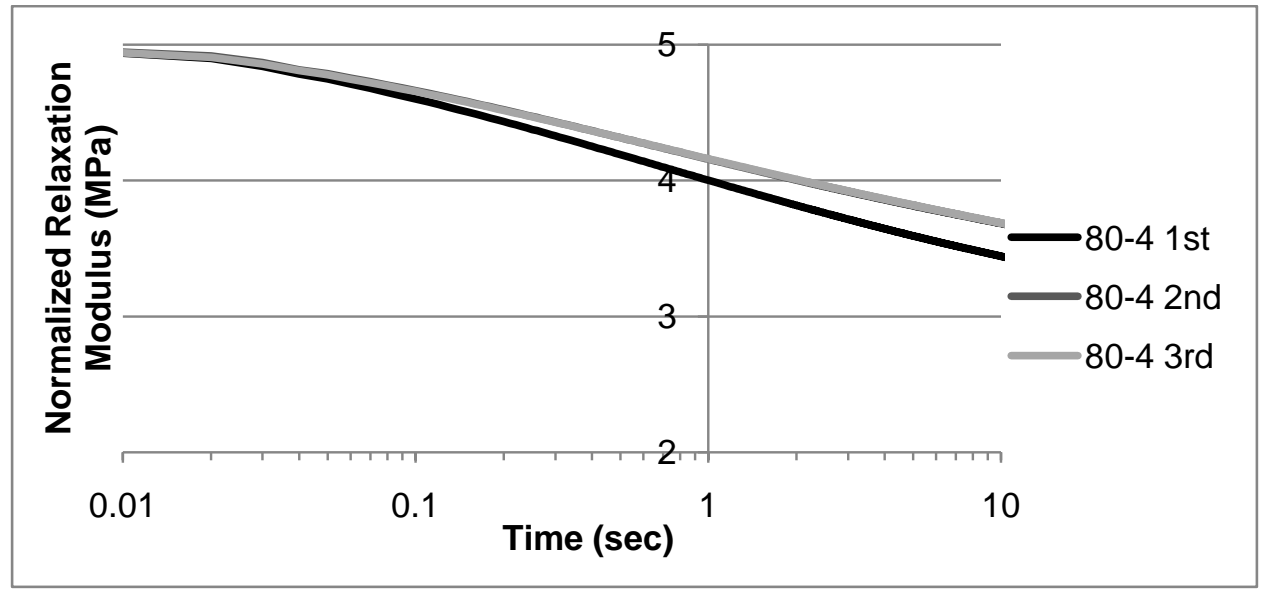

(b)

Figure 2.10 (a) Relaxation Modulus and (b) Normalized Relaxation Modulus for POC-HA 80-4 in Compression Testing

The POC-HA 120-1 material recovered consistently faster than the POC-HA 80-4 which indicates increased elasticity with increased crosslinking time. Before the testing was repeated no signs of permanent deformation were observed in the specimens. Due to the lower initial relaxation moduli upon repeated testing, the test data indicates that there was internal permanent deformation present in the specimen. It was observed that the rate of stress relaxation was faster in the $\mathrm{POC}-\mathrm{HA} 120-1$ material and that the rate of stress decay as shown in Figures $2.10 \mathrm{~b}$ ) and $2.11 \mathrm{~b}$ ) does not change significantly in repeated loading even though the 
starting value is lower. In fact the normalized relaxation rate is slightly higher for both of the materials in the repeated loadings.

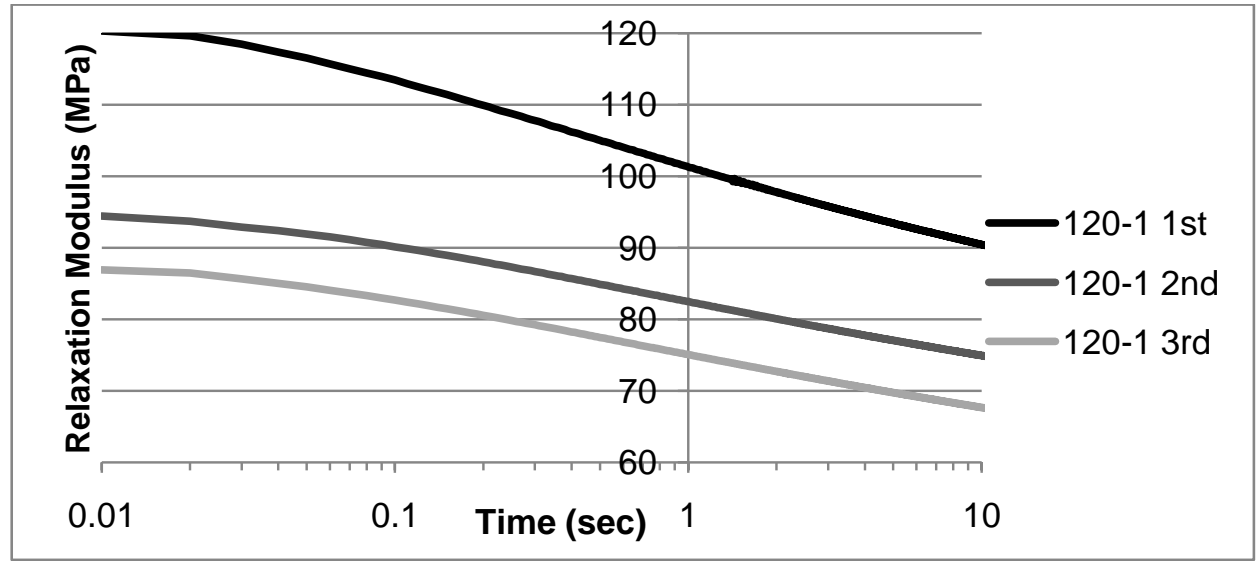

(a)

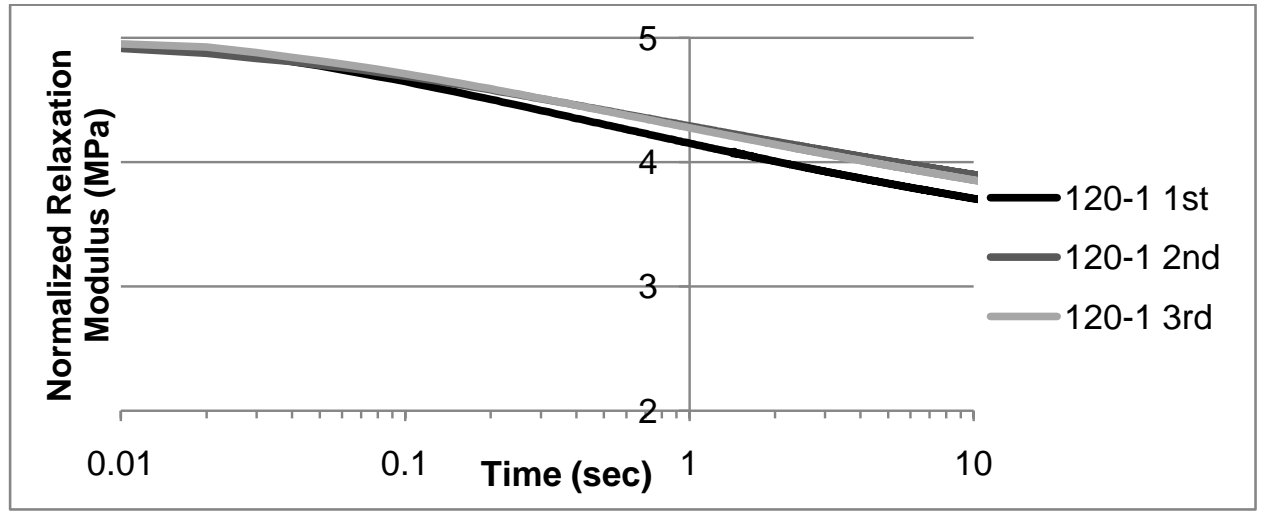

(b)

Figure 2.11 (a) Relaxation Modulus and (b) Normalized Relaxation Modulus for POC-HA 120-1 in Compression Testing

\subsubsection{Cyclic Compression Testing}

To evaluate the material behavior under cyclic loading, a POC-HA 120-1 specimen was cycled with a loading rate of $50 \mathrm{~mm} / \mathrm{min}$ to $20 \%$ strain, held for 30 seconds, released, allowed to recover for 30 seconds, and recompressed. The load was released to $1 \%$ strain to ensure contact of the specimen with the compression plates after release of the load. The degradation 
in the load bearing capacity is presented in Figure 2.12 and the degradation in stiffness per loading cycle is presented in Figure 2.13. The intent of the cyclic testing was to analyze the material behavior during the few initial cycles and not to evaluate the number of cycles the material can withstand until failure.

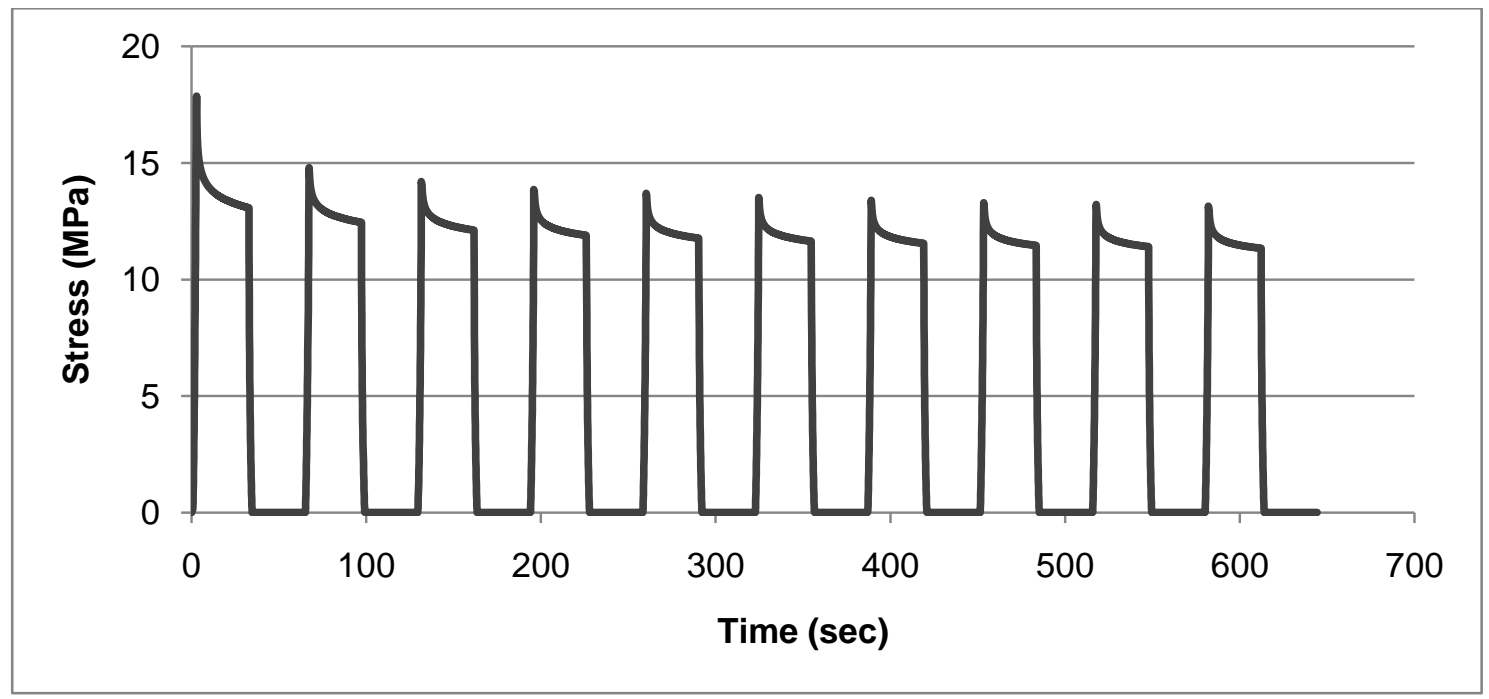

Figure 2.12 Cyclic Compression of POC-HA $120-1$ to $20 \%$ Strain at $50 \mathrm{~mm} / \mathrm{min}$

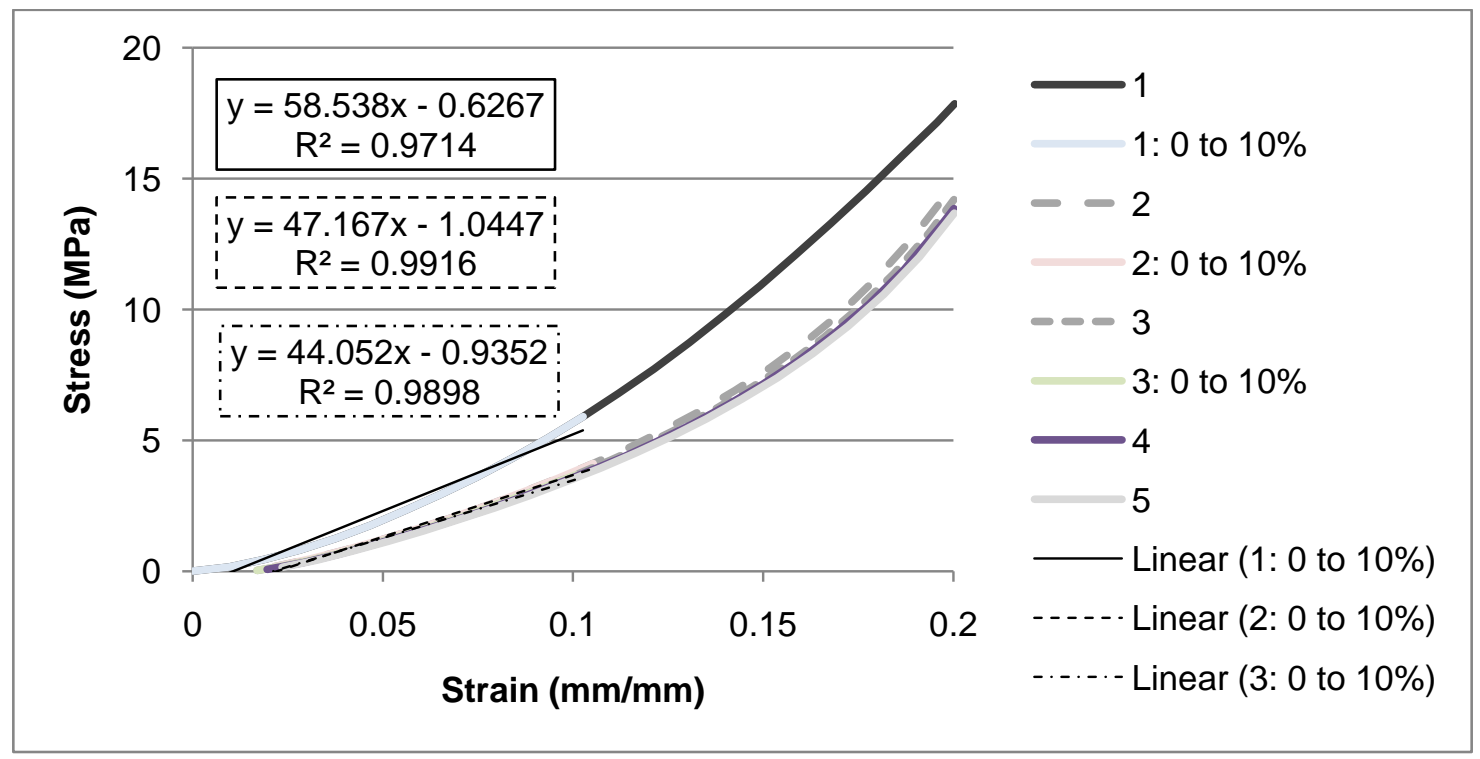

Figure 2.13 Stress-Strain Behavior of POC-HA 120-1 in Cyclic Compression 
In the cyclic compression $25 \%$ of the stiffness (modulus) was lost after the third cycle and $40 \%$ of the load bearing capacity at $10 \%$ strain was lost at the fifth cycle. After the few initial cycles the peak stress seems to be linearly decaying. In a stent application, the first cycle will be the compression for delivery and the second cycle will be caused by the plaque and artery wall. That means POC-HA would be stiffer during delivery than after implantation which is not ideal or desirable behavior. The repeated relaxation experiments show a similar degradation in mechanical properties except the percentages are higher since the material was subject to applied loading for a longer time. The repeated loading causes permanent deformations in the material that may be attributed to realignment of the polymer chains or voids being created in the matrix. The stiffness of the HA phase in the composite is much higher than of the POC phase. At high strains the hard HA particles can shear the POC polymer creating voids.

\subsubsection{Wet Compression Testing}

The material performance and behavior at body conditions needs to be evaluated for stent design. Specimens for both crosslinking conditions ( $\mathrm{N}=3)$, were soaked in $10 \mathrm{~mL}$ of PBS solution (phosphorus buffer solution, $\mathrm{pH}=7.4$ ) at room temperature until mass gain equilibrium was reached. The specimens were weighed every day and placed in $10 \mathrm{~mL}$ of fresh PBS solution. The average mass gain of the specimens is presented in Figure 2.14. The percentage mass gain was calculated by dividing the increase by the original dry specimen mass. The error bars represent the standard deviations in the measurements. At 24 days the POC-HA 80-4 specimens had gained $15.7 \%$ in mass which was associated with a $24.7 \%$ volume increase from dry original dimensions. The POC-HA 120-1 specimens had gained $12.7 \%$ in mass which was associated with a $21.5 \%$ volume increase. The increased crosslinking reduced the swelling behavior of the POC-HA composite. This behavior was expected since there should be less space between the polymer chains for liquid to penetrate. 


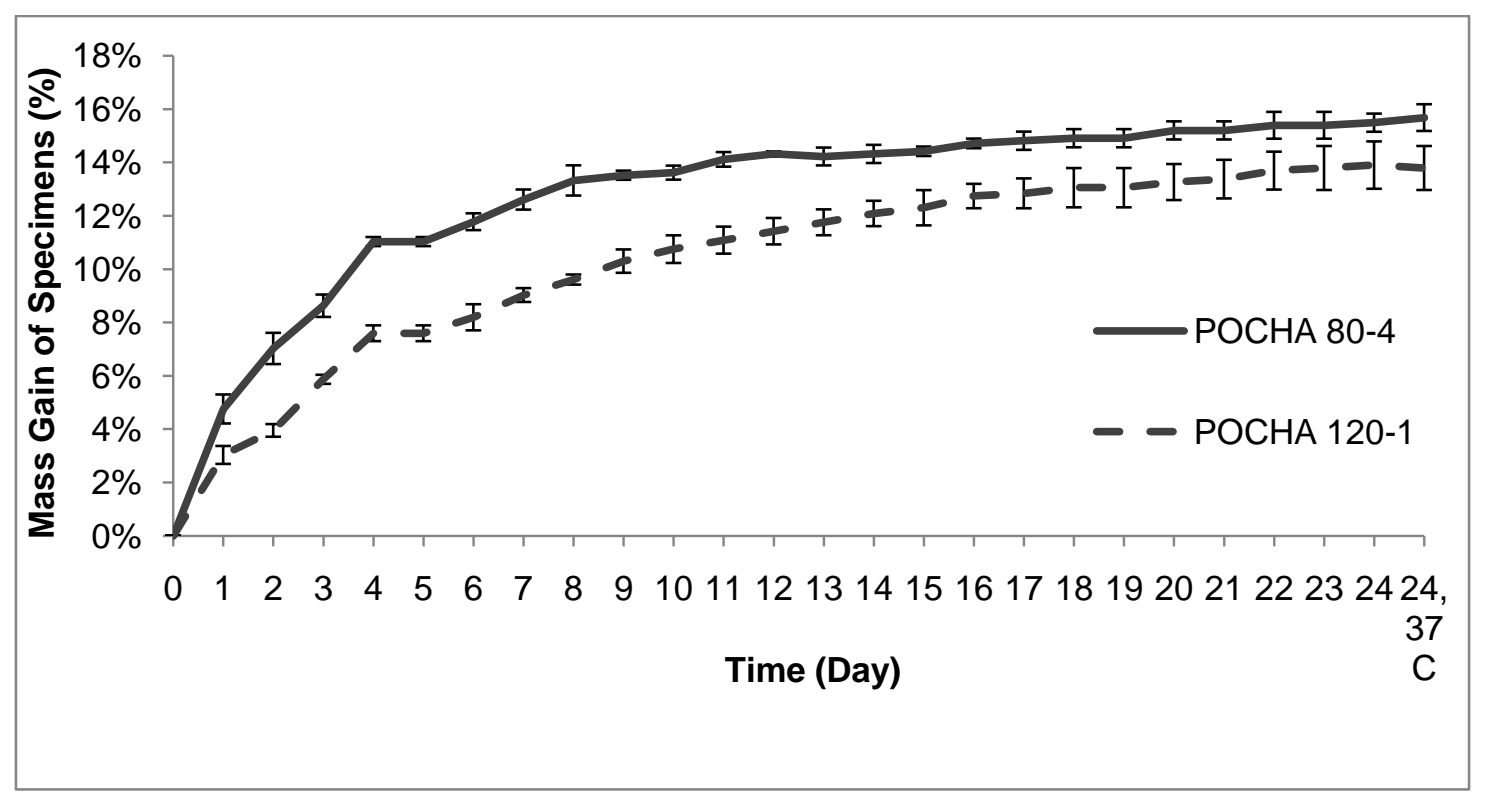

Figure 2.14 Swelling Behavior of the POC-HA Composite

After 24 days the material swelling stabilized and the specimens with $2.5 \mathrm{~mL}$ of PBS were placed in a warm water bath and into a $37^{\circ} \mathrm{C}$ incubator for 30 minutes. The specimens were then loaded axially to failure at a strain rate of $1.5 \mathrm{~mm} / \mathrm{min}$. The comparison of the stressstrain behavior to dry conditions is presented in Figure 2.15. Summaries of the changes in the mechanical properties for each material are presented in Tables 2.3 and 2.4.

No environmental chamber was available during the wet testing to maintain the specimens at constant temperature. Therefore, no stress relaxation testing was performed at emulated body conditions. The specimen temperature after drying the excess PBS from specimen surface, weighing, and photographing had dropped below the $37^{\circ} \mathrm{C}$ before testing. 


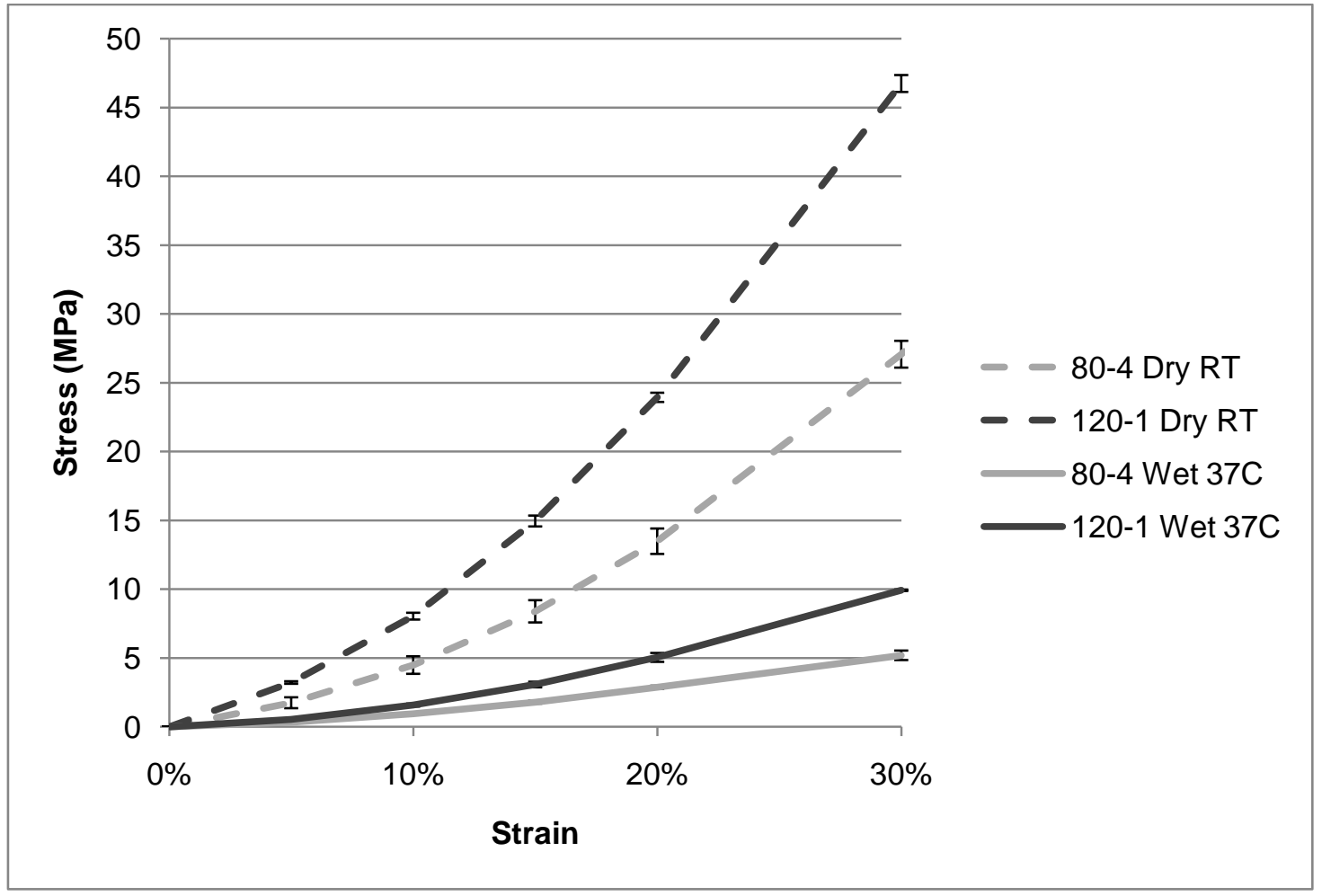

Figure 2.15 Comparison of POC-HA Stress-Strain Behavior for wet and dry Material Conditions

Table 2.3 Effect of Body Conditions on POC-HA 80-4

\begin{tabular}{|c|c|c|c|c|c|c|c|c|}
\hline \multirow[b]{2}{*}{ Condition } & \multicolumn{2}{|c|}{ Young's Modulus (MPa) } & \multicolumn{5}{|c|}{ Stress (MPa) at Strain (\%) } & \multirow[b]{2}{*}{$\begin{array}{l}\text { Max. } \\
\text { strain }\end{array}$} \\
\hline & $\begin{array}{l}0-10 \% \\
\text { Strain }\end{array}$ & $\begin{array}{l}10-20 \% \\
\text { Strain }\end{array}$ & $5 \%$ & $10 \%$ & $15 \%$ & $20 \%$ & $30 \%$ & \\
\hline Dry RT & 45.59 & 92.33 & 1.75 & 4.49 & 8.39 & 13.49 & 27.08 & $50 \%$ \\
\hline Wet 37C & 9.83 & 19.19 & 0.34 & 0.96 & 1.79 & 2.88 & 5.20 & $30 \%$ \\
\hline \% Change: & -78.43 & -79.21 & -80.86 & -78.71 & -78.62 & -78.67 & -80.80 & -39.45 \\
\hline
\end{tabular}

Table 2.4 Effect of Body Conditions on POC-HA 120-1

\begin{tabular}{|c|c|c|c|c|c|c|c|c|}
\hline \multirow[b]{2}{*}{ Condition } & \multicolumn{2}{|c|}{ Young's Modulus (MPa) } & \multicolumn{5}{|c|}{ Stress (MPa) at Strain (\%) } & \multirow[b]{2}{*}{$\begin{array}{l}\text { Max. } \\
\text { strain }\end{array}$} \\
\hline & $\begin{array}{l}0-10 \% \\
\text { Strain }\end{array}$ & $\begin{array}{l}10-20 \% \\
\text { Strain }\end{array}$ & $5 \%$ & $10 \%$ & $15 \%$ & $20 \%$ & $30 \%$ & \\
\hline Dry RT & 79.61 & 159.84 & 3.22 & 8.04 & 14.96 & 23.94 & 46.75 & $46 \%$ \\
\hline Wet 37C & 16.02 & 34.75 & 0.55 & 1.58 & 3.07 & 5.05 & 9.92 & $33 \%$ \\
\hline$\%$ Change: & -79.88 & -78.26 & -82.93 & -80.35 & -79.45 & -78.93 & -78.77 & -28.36 \\
\hline
\end{tabular}


The body conditions have a major effect on the mechanical properties of the POC-HA material. For both of the crosslinking conditions, the elastic moduli as well as the load bearing capacity were reduced by $80 \%$ as compared to the dry material at room temperature. Similar behavior has been observed in polymeric materials in experiments by Wu et. al. where PLGA porous scaffolds lost about $90 \%$ and PDLLA porous scaffolds lost 20 to $25 \%$ of their mechanical properties after 2 to 4 hours of wetting in PBS at $37^{\circ} \mathrm{C}[\mathrm{Wu}, 2006]$. Obviously there is porosity in the POC-HA composite which allows for water absorption which weakens the material and creates brittleness. During the testing it was observed that the original material was hard while the wet material felt spongy when compressed with fingers. After compression testing there was no significant weight loss in the specimen, only about one percentage point. This suggests that the water bonded into the matrix or the material swelling had not reached an equilibrium level. Pure hydroxyapatite $(\mathrm{HA})$ is known to hydrolyze water molecules. To reduce this swelling behavior some compound should be added to POC-HA or different crosslinking condition should be investigated to develop a more hydrophobic material. In the wet testing all specimens shattered into pieces which could be problematic for a stent application since broken pieces can travel with the bloodstream unless the stent is already covered with endothelial cells.

SEM images of failed dry and wet POC-HA 120-1 compression specimens are presented in Figures 2.16 and 2.17 respectively. The images show the outside cylindrical face of the specimen at a failure region. For the dry specimen (Figure 2.16) the failure was a surface crack near the top of the specimen. The area on the right is the outside surface. The specimen outside surfaces were not smooth but had cavities which was an artifact of the molding technique. The wet testing specimen failed at the center. The bottom of Figure 2.17 shows the specimen outside surface and the top of the image covers the area where the material failed. It was observed that the failed specimen from wet testing had more visible voids inside. 


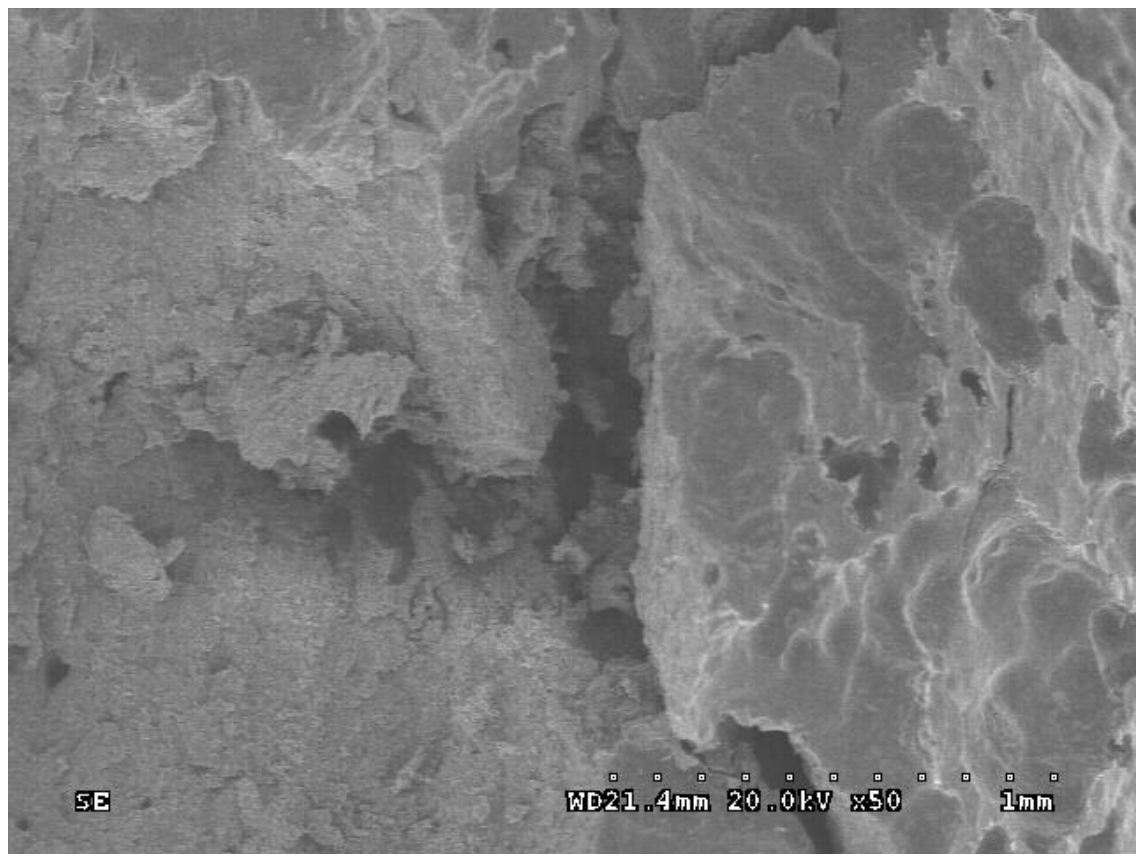

Figure 2.16 Surface of a dry POC-HA 120-1 Compression Specimen at Failure Region (scale bar 1 millimeter)

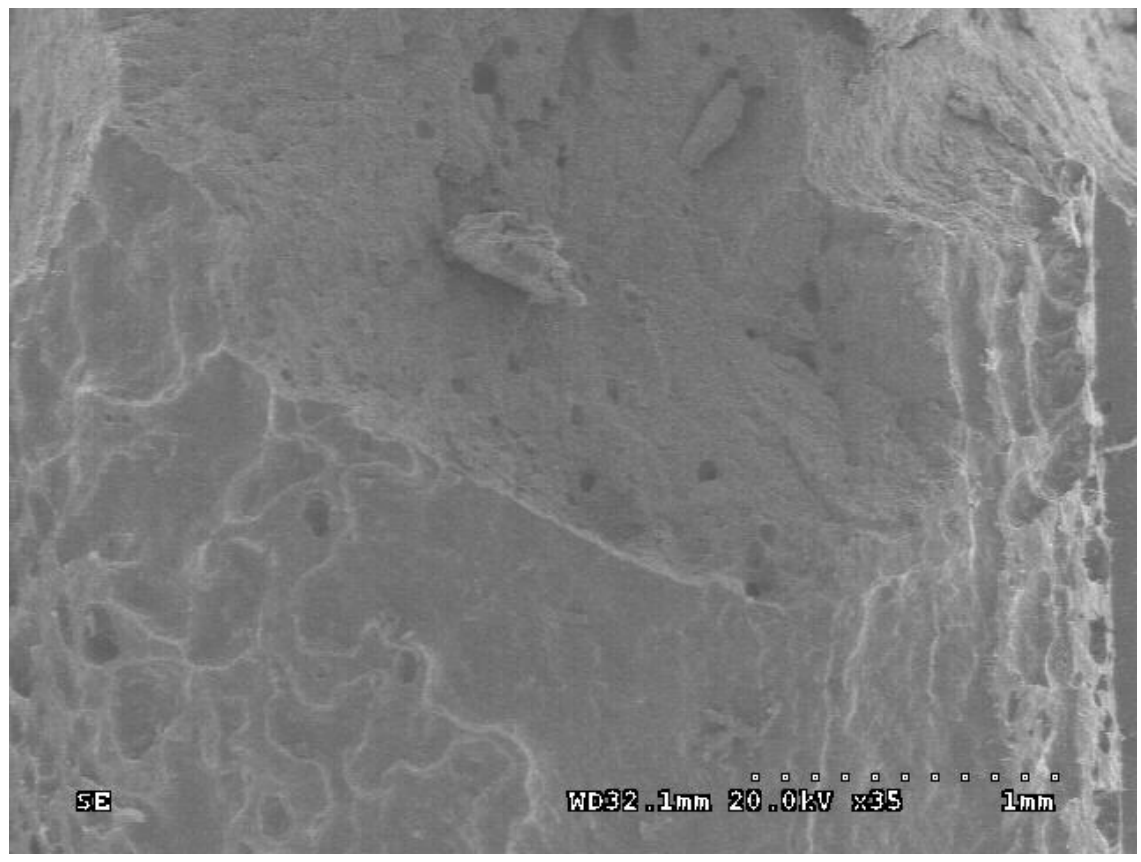

Figure 2.17 Surface of a POC-HA 120-1 Compression Specimen after wet Mechanical Testing (scale bar 1 millimeter) 


\section{CHAPTER 3}

\section{FABRICATION}

\subsection{Femtosecond Laser Fabrication}

Femtosecond lasers have been used in micro fabrication for the past twenty years with success. The characteristic of this type of laser is their short pulse duration. The pulse energy is concentrated in the pulse width of $\sim 120$ fs (femtoseconds) which causes material ablation in a process called cold-ablation resulting in minimal heat affected zone (HAZ) around the ablated area [Uppal, 2005]. This ensures uniform mechanical properties in the machined stent struts as well as preventing melting and resolidification which could affect the mechanical properties and degradation rate of the material.

The micromachining setup utilized is the equivalent of a 4-axis CNC machine (3 linear and one rotary axis). The femtosecond laser micromachining setup includes a femtosecond laser from Spectra-Physics with controlled pulse rate of up to $1 \mathrm{kHz}$ and controlled power through an attenuator of up to $700 \mathrm{~mW}$ per pulse. A set of optics guides the laser beam which acts as the machine tool to the material for a subtractive machining process. The laser energy can be focused in the $\mathrm{XYZ}$ space and the POC-HA tubes are mounted on the rotary axis which is in the XY plane. The focused femtosecond laser beam causes ablation of the material if the energy is above the threshold fluence. The material is converted straight into plasma. Since the pulses are short, heat transfer to the area near the cut does not occur resulting in a clean cut with no cracks on the surface and with minimal heat affected zone. The surface cracks might degrade the structural integrity of the stent material especially during repeated pulsation in the blood vessel. 


\subsection{Ablation Behavior of POC-HA}

The ablation studies indicate that clean cuts can be achieved with relatively high power levels with POC-HA at both of the crosslinking conditions presented. It is observed that no melting and resolidification or surface cracks are generated around the ablated spots and the walls of a machined hole are smooth as shown in Figures 3.1 and 3.2. The SEM images shown are from POC-HA 80-4 material. Power levels of around $300 \mathrm{~mW}$ were used with a $25.4 \mathrm{~mm}$ focal length lens to fabricate stent geometries from POC-HA tubes. Along with feed rates that provide for $80 \%$ spot overlap a consistent material removal was achieved up to 30 micron depth for POC-HA 80-4 and 20 micron depth for POC-HA 120-1 per machining pass.

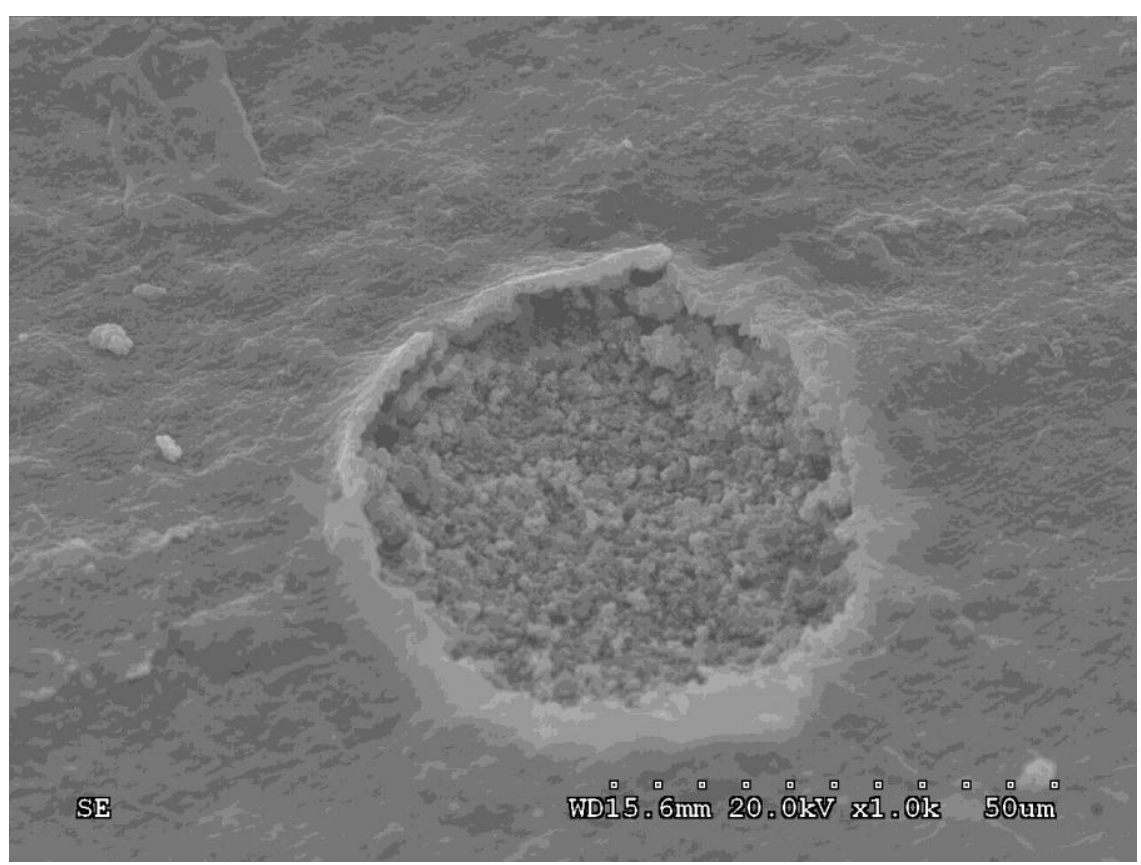

Figure 3.1 Ablated Surface with 10 Pulses at $220 \mathrm{~mW}$ (scale bar 50 microns) 


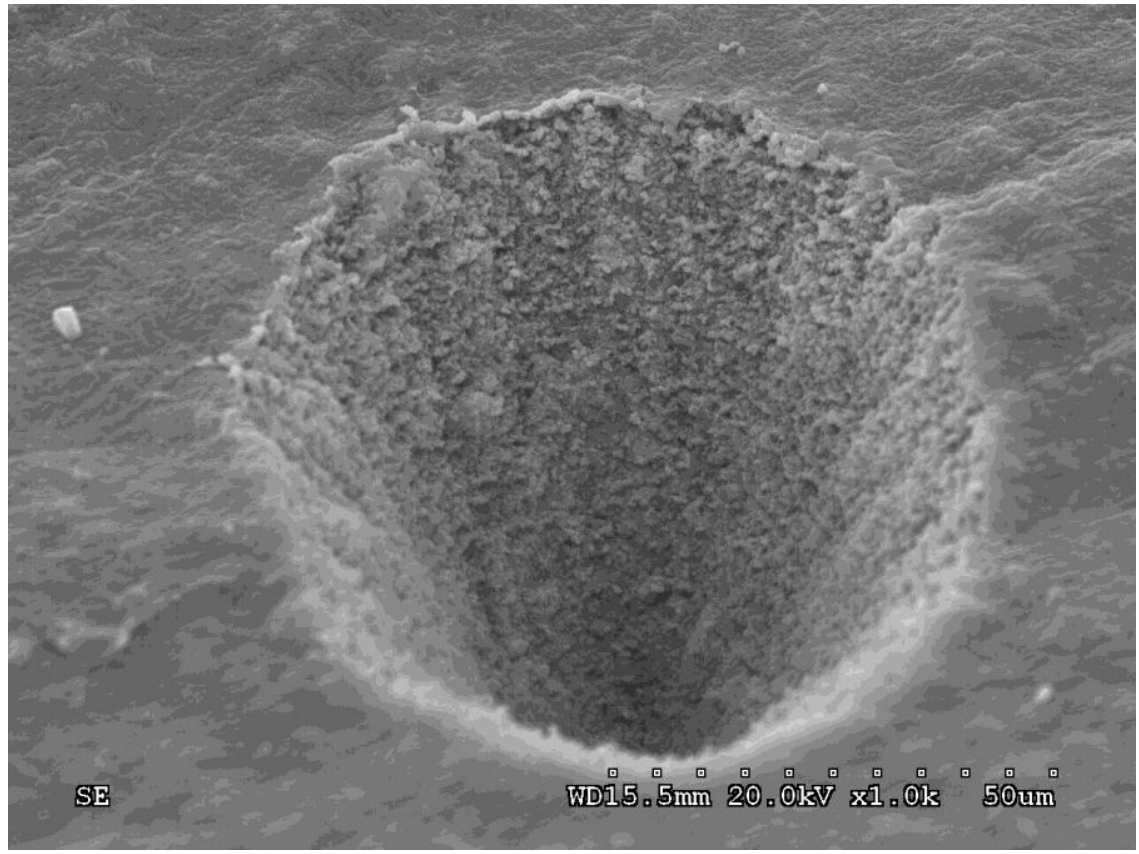

Figure 3.2 Ablated Surface with 100 Pulses at $220 \mathrm{~mW}$ (scale bar 50 microns) 


\section{CHAPTER 4}

\section{DESIGN AND ANALYSIS}

Mechanical properties of biodegradable polymers are inferior compared to metals. Therefore the design of the stent geometry or pattern needs to be highly optimized [Grabow, 2007]. A suitable tool for designing optimized structures is finite element analysis. The FEA method solves the displacements of a discretized (meshed) model based on a constitutive equation and applied loads and boundary conditions. ANSYS ${ }^{\circledR} 11.0$ finite element analysis software was utilized to create simulation models for POC-HA material. First analyses were performed that replicated the mechanical testing cases to verify the accuracy of the developed material model. The quality of the finite element solution is dependent on the specified material properties and the selected material models. Then the developed models were used to evaluate the load bearing capacity of the POC-HA material for a coronary stent application. The focus of the finite element analysis was on the POC-HA 120-1 material due to its better mechanical properties based on testing results.

\section{$\underline{4.1 \text { Finite Element Analysis Techniques }}$}

The geometries for finite element studies were created using Pro/ENGINEER ${ }^{\circledR}$ Wildfire 3.0 solid modeling software. For stent modeling, planar strut sketches were wrapped onto a cylindrical surface to create the edges for stent pattern. The wrapped sketches were used to trim the curved surface which was then thickened to create a 3-D stent model. The projected edges can also be used to create the G-codes used in the laser fabrication.

The original stent solid model patterns developed in Pro/ENGINEER were imported in ANSYS for finite element analysis. It was found that the import of complex geometries with intersecting surfaces resulted in models which could not be meshed cleanly and properly with 
ANSYS mesh generation algorithms. In ANSYS, there are not many tools for geometry cleanup and manual mesh generation which made this important step a very time consuming task. This difficulty was overcome by employing a different pre-processing CAE tool, HyperMesh ${ }^{\circledR} 9.0$ by Altair Engineering Inc., to prepare the models for solving in ANSYS. The solid 3-D stent geometries developed in Pro/ENGINEER were imported into HyperMesh where the cylindrical surfaces were meshed successfully. From the surface elements a selected number of elements were extruded through the stent thickness. HyperMesh also has tools to check the various element quality criteria to ensure well behaving solutions. Element definitions, material property assignments and boundary conditions can also be applied in HyperMesh during the preprocessing stage and included in the model exported to ANSYS.

HyperMesh allows for better geometry cleanup and more efficient mesh generation than ANSYS for the intricate stent geometries. The developed models can also be exported and used in other commercial finite element codes for other types or more specialized analyses as needed. The main steps in the creation of a finite element model for a stent pattern in contact with the artery wall which transmits the pressure loading are presented in Figure 4.1.

Pre-processing for the simpler verification models were also performed in HyperMesh. These models have the same geometry but different boundary conditions and material properties. The ANSYS database files can be exported from a single meshed model by altering the boundary conditions and material properties as needed. The models can also be easily prepared for results extraction in ANSYS during the preprocessing stage in HyperMesh. Renumbering the nodes of interest in consecutive order makes the preparation of ANSYS command scripts easier. For example picking the 53 required nodes for results extraction on the tensile strip model by hand in ANSYS GUI would have taken a considerable amount of time. When the nodes are ordered consecutively from 1 to 53 , a command line can be created in for example in Excel and iterated for all nodes to make a single command script to assign all of the nodes at once. 


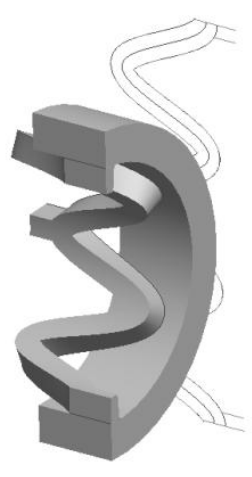

(a)

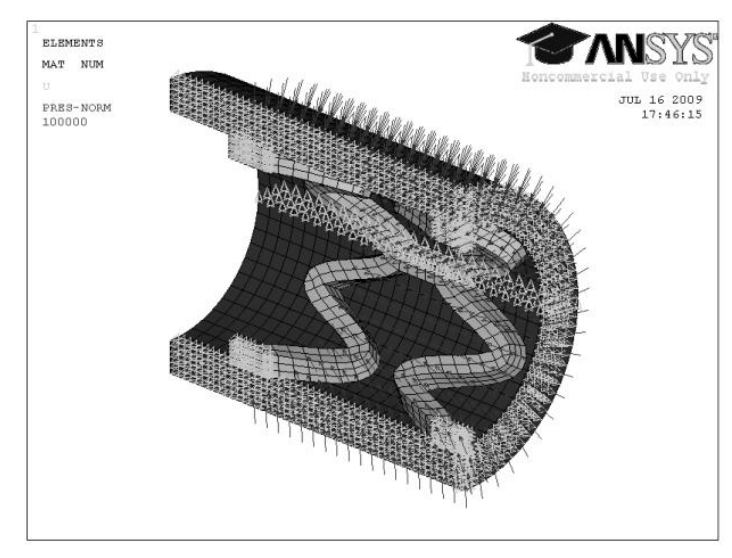

(c)

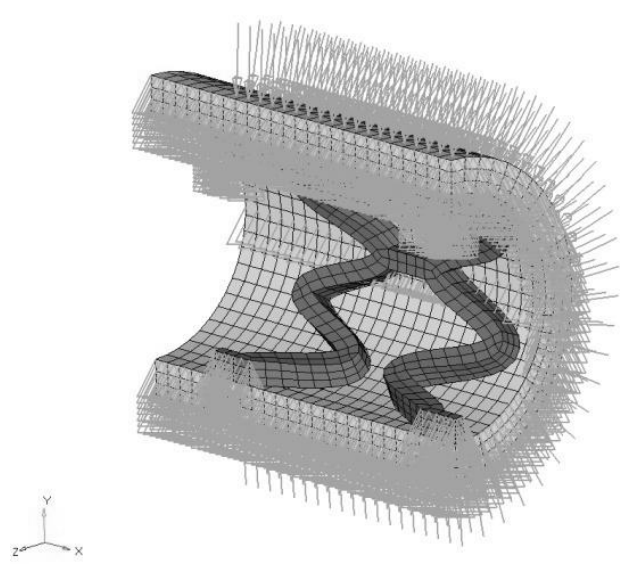

(b)

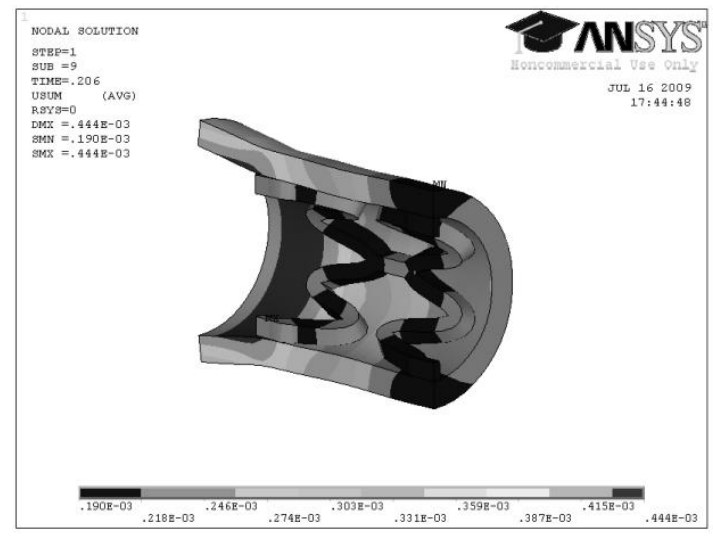

(d)

Figure 4.1 Stent Finite Element Model Creation Steps: (a) Strut Pattern Generated in Pro/ENGINEER, (b) Generated Mesh with Element Definitions, Material Properties, Boundary Conditions and Loading Specified in HyperMesh, (c) Imported Finite Element Model in ANSYS, and (d) Displacement Solution in ANSYS

\section{$\underline{4.2 \text { Verification of Material Models }}$}

The ANSYS element type used for all analyses was SOLID186. This is a 20 node brick element which includes a quadratic shape function and material nonlinearities such as viscoelasticity, yielding, and hyperelasticity. The material model used was linear elastic with viscoelastic behavior. The Young's modulus used was the 0 to $10 \%$ strain range least squares linear fit obtained from mechanical testing of the material. This strain range was selected since 
the largest diameter change that an artery experiences in a cardiac cycle is around $10 \%$ [Ozolanta, 1998]. Therefore, the strains in the stent should not exceed $10 \%$. In this strain range the POC-HA material has a linear behavior and the material model should be accurate for stent design based on experimental data. For Poisson's ratio the values obtained from tensile testing were used.

The viscoelastic effect for SOLID186 is incorporated using a Prony series approximation [ANSYS]. This model is a decaying function of time which reduces modulus from the specified shear modulus. It was observed in the repeated relaxation testing that the rate of stress relaxation does not change significantly with repeated loading. Therefore a single decaying function can capture the behavior accurately. The formulation of the Prony series approximation is shown in Equation 1, where $\mathrm{G}_{\infty}$ represents instantaneous shear modulus which is assigned by the elastic modulus, t represents time, and $G_{i}$ represents coefficient and $T_{i}$ represents time constant for the series terms [ANSYS].

$$
G=G_{\infty}+\sum_{i=1}^{n} G_{i} e^{\left(-\frac{t}{\tau_{i}}\right)}
$$

The stress relaxation modulus data obtained from the mechanical testing was converted into shear modulus using the Equation 2:

$$
\mathrm{G}=\frac{\mathrm{E}}{2(1-v)}
$$

The fit used was a four term approximation for experimental data up to 10 seconds. In the actual stent loading the relaxation time will be in the order of milliseconds assuming a heart rate of $1 \mathrm{~Hz}$. A curve fitting tool is available in ANSYS which solves for the coefficients of the series terms in Equation 1 from a specified data set. The time constants chosen for the terms were $0.01,0.1,1$, and $10 \mathrm{sec}$ following a logarithmic spacing. The Prony series fit for the POCHA 120-1 material in compression as generated in ANSYS is shown in Figure 4.2. The quality of 
the fit was similar for all of the four relaxation cases. A good fit was obtained for the compression cases if the time points for series expansion were fixed at the specified values when solving the series coefficients.

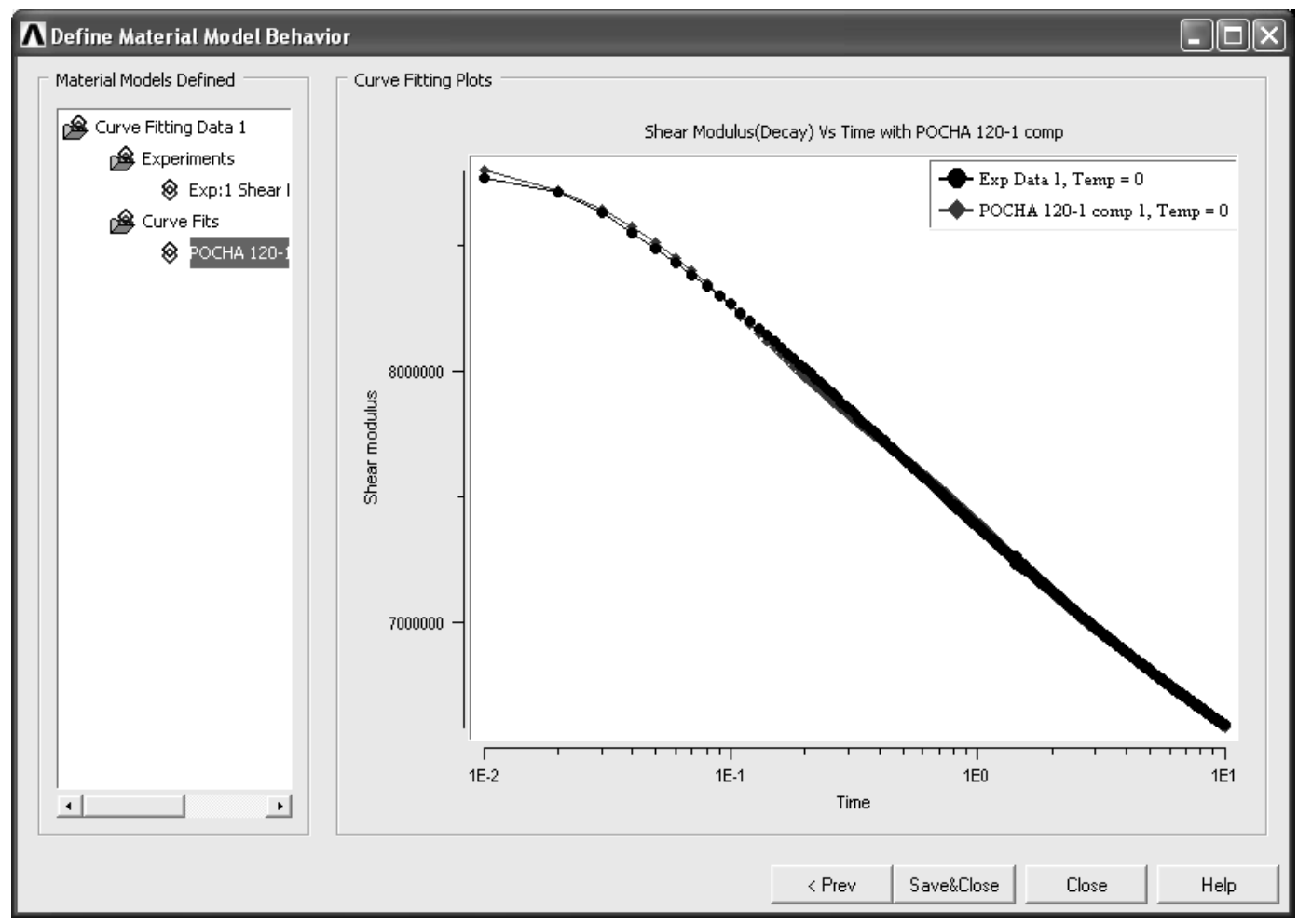

Figure 4.2 ANSYS Prony Series Fit for POC-HA 120-1 Stress Relaxation in Compression

In the mechanical testing the end conditions of the sample are not ideal. In compression testing there is friction at the interfaces of the specimen and the compression plates which leads to bulging at the center of the specimen. In tensile testing the grips restrict displacement in the lateral direction and the width reduction of the specimen may not be uniform. Therefore for material model verification two scenarios, free and fixed lateral displacements at specimen ends, were analyzed to determine which model follows the mechanical test data more accurately. 


\subsubsection{Tensile Model}

The finite element model used for the tensile testing was a full length half model using width symmetry. The model dimensions were $3 \mathrm{~mm}$ width, $1 \mathrm{~mm}$ thickness, and $60 \mathrm{~mm}$ length. The bottom nodes were fixed in all directions for fixed case and in Z-direction for free case. The symmetry conditions of fixed X-displacements were at the respective cut face. At the top surface the nodes were numbered for easier results extraction, constraints were specified for free or fixed case, and assigned a velocity of $8.3 \mathrm{E}-4$ or $8.3 \mathrm{E}-3 \mathrm{~m} / \mathrm{s}$, velocity levels corresponding to crosshead speeds of $1.5 \mathrm{~mm} / \mathrm{min}$ or $50 \mathrm{~mm} / \mathrm{min}$ respectively. The model was analyzed for a transient loading with all initial conditions at zero and constant velocity until the final time to reach a $20 \%$ strain with a step size of 0.1 seconds. For stress relaxation verification the constant velocity was applied until 0.2 seconds less than the time required for the $10 \%$ strain to account for the deceleration of the MTS machine before the maximum strain was reached, after which a load step with zero velocity was applied for 10 seconds. In the TimeHist post-processing the values for Z-displacement at one of the nodes and Z-direction reaction forces at all of the nodes on the top surface were extracted to compare the stress-strain behavior of the finite element solution to experimental data. The tensile finite element model in HyperMesh is shown Figure 4.3.

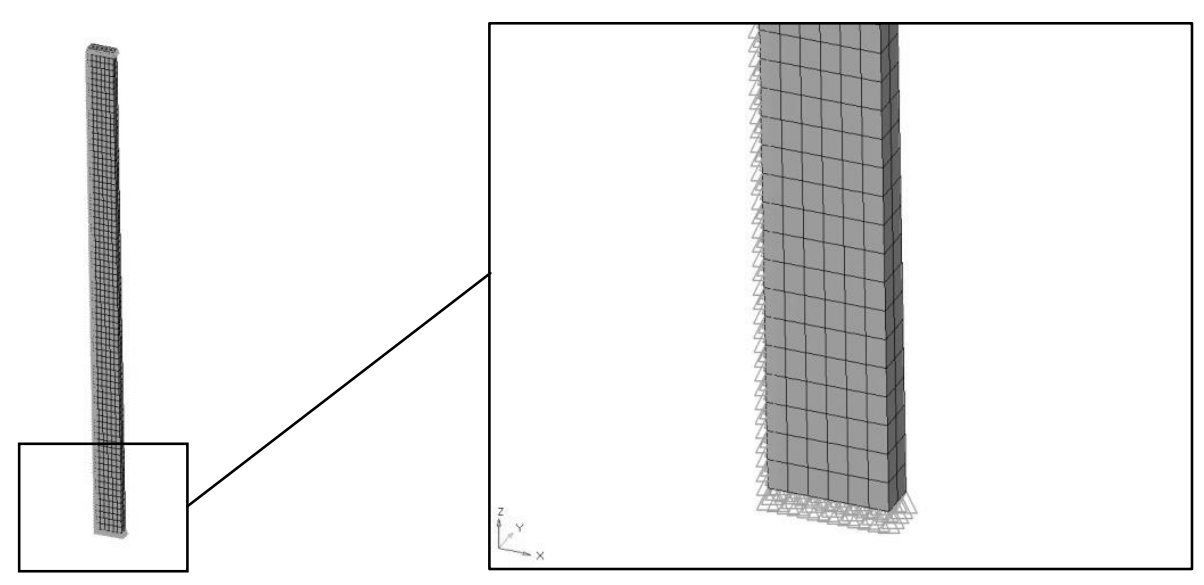

Figure 4.3 Tensile Verification Finite Element Model 
The stress-strain behavior for the two materials (POC-HA 80-4 and POC-HA 120-1) from the tensile finite element models and the corresponding experimental data are shown in Figures 4.4 and 4.5 . The stress from the finite element solution was calculated by dividing the sum of the axial reaction forces at the specimen end nodes by the original cross section area at each of the solved time points. The MTS curves were plotted from averaged stress values at strain points from the experimental testing data. The error bars represent the standard deviation in the testing data.

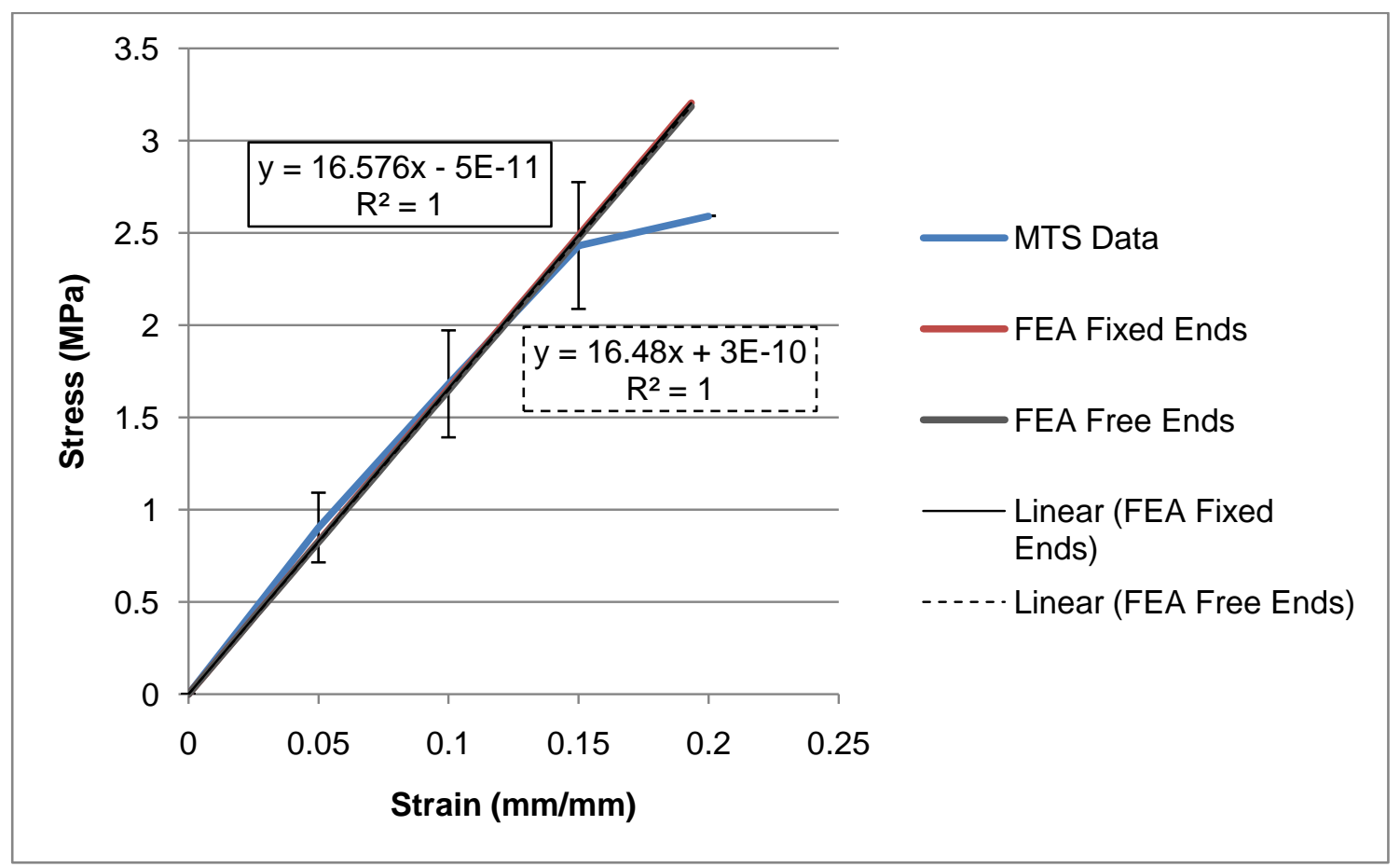

Figure 4.4 Comparison of POC-HA 80-4 Material Models with Experimental Tensile Data 


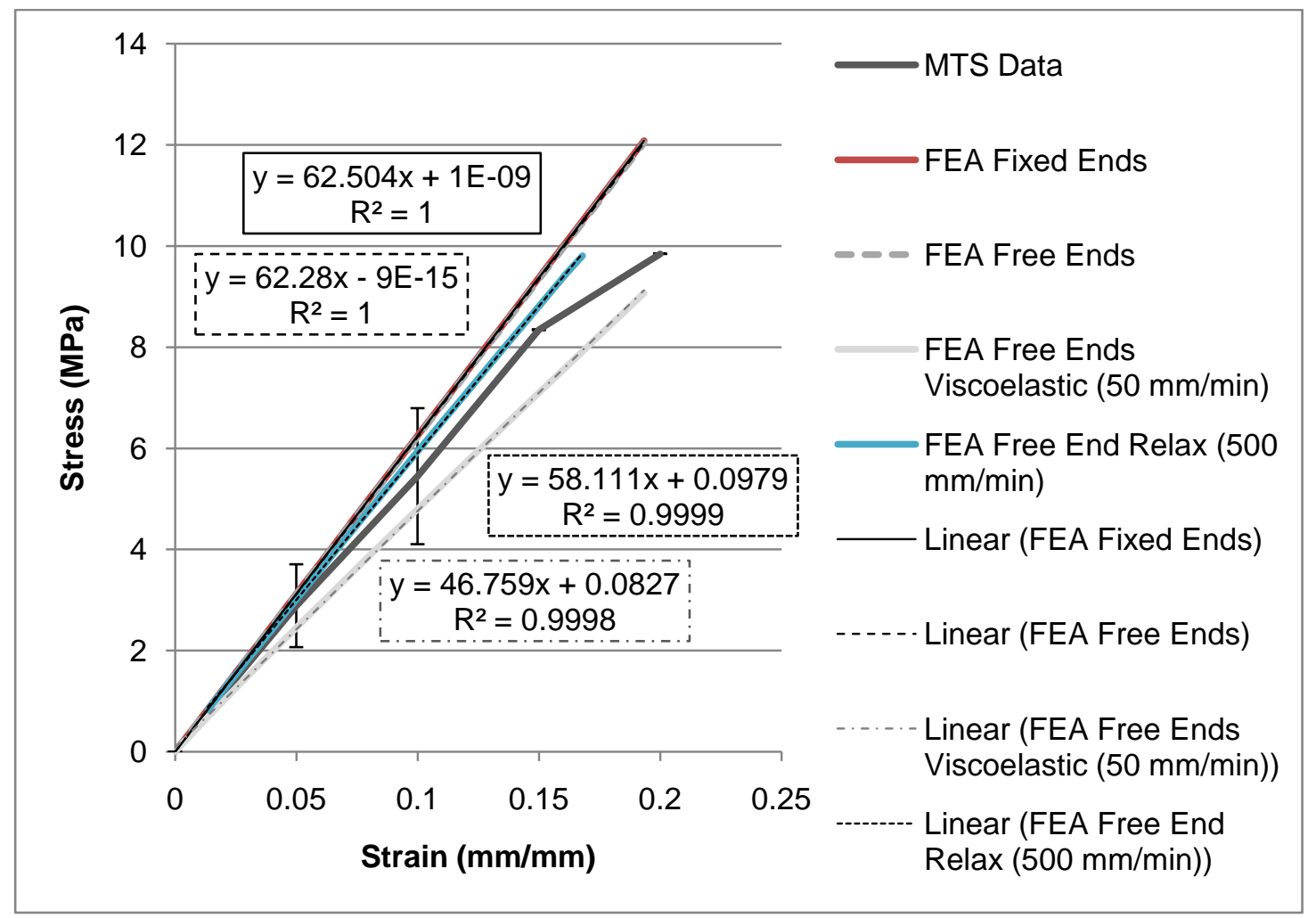

Figure 4.5 Comparison of POC-HA 120-1 Material Models with Experimental Tensile Data

Based on Figures 4.4 and 4.5 the linear elastic model captured the mechanical testing behavior accurately in the 0 to $10 \%$ strain range which is of interest in stent design. The linear fit was applicable up to $15 \%$ strain after which the data deviates as expected since the material model was fitted in the 0 to $10 \%$ strain range. A modulus of $6.228 \mathrm{E} 7 \mathrm{~Pa}$ was used instead of 4.979E7 Pa (the average from tensile testing) for POC-HA 120-1 tensile verification model. This would be the average if the less stiff linear behavior was ruled as an outlier due to possible specimen defects or slippage in testing. The finite element data in Figure 4.5 is still within the standard deviation of the mechanical testing. The difference in the finite element model end conditions did not have a significant effect on the solution results. This can be explained by the large aspect ratio of the specimen which ensured that the behavior at the specimen ends did not affect global results. It is also observed that the specified viscoelastic model was able to 
capture the strain rate effect as shown in Figure 4.5. There was a drop in the measured modulus when the strain rate was reduced.

The stress relaxation of the tensile verification models is shown in Figure 4.6. For both of the materials the actual specimen relaxed faster than the finite element solution. Based on the normalized stress decay in Figure 4.7 the relaxation was $10 \%$ in the finite element solution and around $18 \%$ in the actual test specimen after 10 seconds of constant applied strain. The decay is faster in the actual specimen during the first second after which the slopes equalize.

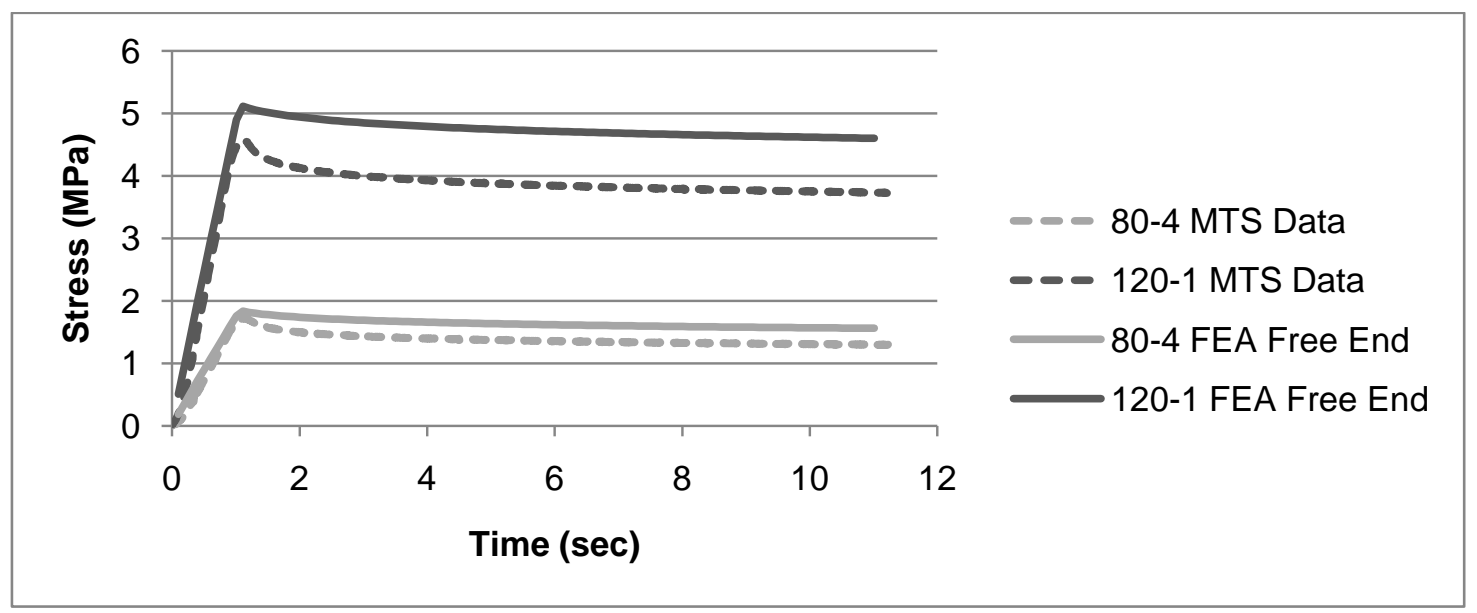

Figure 4.6 Comparison of Stress Relaxation Models to Experimental Tensile Data

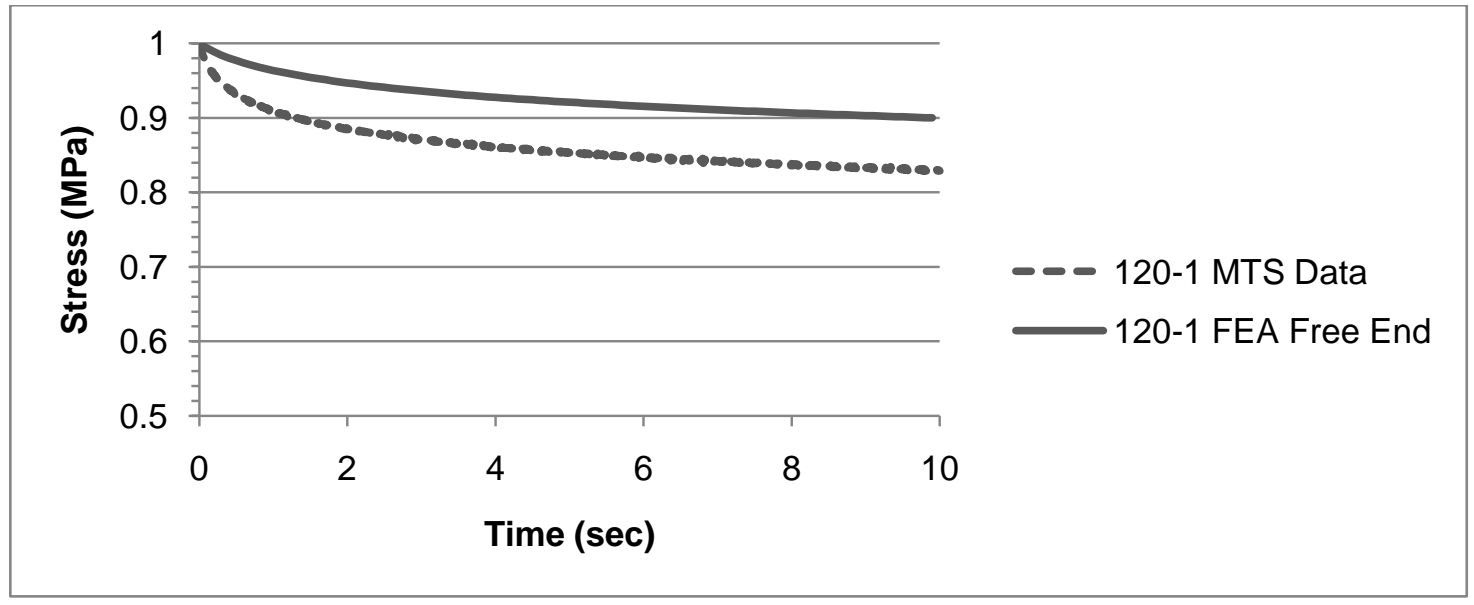

Figure 4.7 Normalized Stress Relaxation for POC-HA 120-1 in Tension 
The reasons for this discrepancy could be attributed to the material testing conditions, specified solution controls, and selected viscoelastic model. In the relaxation testing the strain application should be instantaneous to ensure that no relaxation occurs during the loading of the specimen. This cannot be achieved with the available screw driven MTS testing machine. The relaxation that occurred in the specimen during the 1.2 seconds loading was not captured in the relaxation data and therefore not present in the viscoelastic model. In stent application the interest is on the relaxation that occurs immediately after the applied loading and before the next cyclic loading.

The specified time step and solver type affect the finite element solution. Small displacement transient with automatic time stepping was used in the solutions. The small displacement assumption should be valid in the $10 \%$ strain range. The Prony series viscoelastic formulation is also based on the small strain assumption and corresponds to the classical parallel Maxwell model [ANSYS].

The material properties used in the tensile verification models are summarized in Tables 4.1 and 4.2. EX being the Young's modulus, PRXY being the Poisson's ratio and all of the coefficients are in metric units. The a3 and 3 coefficients for POC-HA 80-4 indicate that the fit could have been reasonably achieved using a three term approximation.

Table 4.1 Material Properties for POCHA 80-4 ANSYS Tensile Model (Metric Units)

\begin{tabular}{|c|c|}
\hline \multicolumn{2}{|c|}{ Linear Elastic Isotropic } \\
\hline EX (Pa) & $1.721 \mathrm{E} 7$ \\
\hline PRXY & 0.39 \\
\hline Nonlinear Viscoelastic & Prony Shear Response \\
\hline a1 & 0.076907 \\
\hline $\mathrm{t} 1$ & 0.85256 \\
\hline $\mathrm{a} 2$ & 0.12653 \\
\hline $\mathrm{t} 2$ & 5.9988 \\
\hline $\mathrm{a} 3$ & $4.2107 \mathrm{E}-14$ \\
\hline $\mathrm{t} 3$ & -82.105 \\
\hline $\mathrm{a} 4$ & 0.057103 \\
\hline $\mathrm{t} 4$ & 0.17962 \\
\hline
\end{tabular}


Table 4.2 Material Properties for POCHA 120-1 ANSYS Tensile Model (Metric Units)

\begin{tabular}{|c|c|}
\hline \multicolumn{2}{|c|}{ Linear Elastic Isotropic } \\
\hline EX $(\mathrm{Pa})$ & $6.228 \mathrm{E} 7$ \\
\hline $\mathrm{EX}$ (average) $(\mathrm{Pa})$ & $4.979 \mathrm{E} 7$ \\
\hline PRXY & 0.31 \\
\hline Nonlinear Viscoelastic Prony Shear Response \\
\hline $\mathrm{a} 1$ & 0.092929 \\
\hline $\mathrm{t} 1$ & 10.634 \\
\hline $\mathrm{a} 2$ & 0.055051 \\
\hline $\mathrm{t} 2$ & 1.5414 \\
\hline $\mathrm{a} 3$ & 0.039978 \\
\hline $\mathrm{t} 3$ & 0.37851 \\
\hline $\mathrm{a} 4$ & 0.017975 \\
\hline $\mathrm{t} 4$ & 0.08976 \\
\hline
\end{tabular}

\subsubsection{Compression Model}

The model used for the compression testing was quarter cylinder geometry with full length taking advantage of the symmetry. The cylinder dimensions were $4.45 \mathrm{~mm}$ in diameter and $9.65 \mathrm{~mm}$ in length. The bottom nodes were fixed in all directions for fixed case and in Zdirection for free case. The symmetry conditions of fixed $\mathrm{X}$ - and $\mathrm{Y}$-displacements were applied to the respective cut faces. At the top surface the nodes were numbered for easier results extraction, constraints were specified for free or fixed case, and assigned velocities of $-2.5 \mathrm{E}-5$ $\mathrm{m} / \mathrm{s}$ and $-8.3 \mathrm{E}-4 \mathrm{~m} / \mathrm{s}$ corresponding to crosshead speeds of $1.5 \mathrm{~mm} / \mathrm{min}$ and $50 \mathrm{~mm} / \mathrm{min}$ respectively. The models were analyzed for a transient loading with all initial conditions at zero and constant velocity until final time to reach a $20 \%$ strain with a step size of 0.1 seconds. The steps for results extraction and stress calculation from results were same as in tensile verification model cases. 


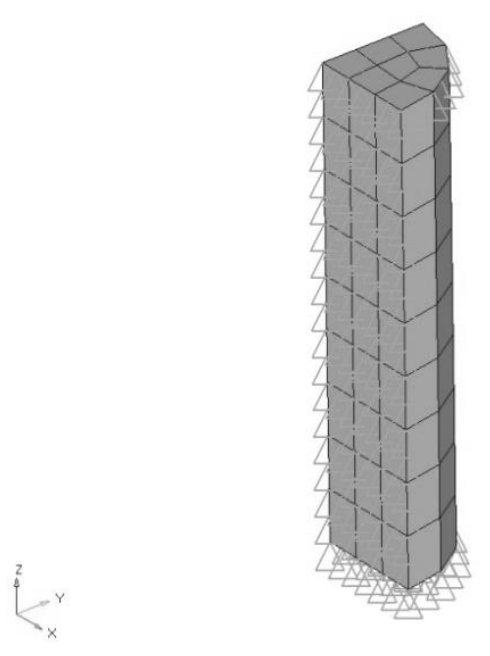

Figure 4.8 Compression Verification Finite Element Model

The stress-strain behavior for the two materials (POC-HA 80-4 and POC-HA 120-1) from the compression finite element models and the corresponding experimental data are shown in Figures 4.9 and 4.10. The error bars represent the standard deviation in the MTS testing data.

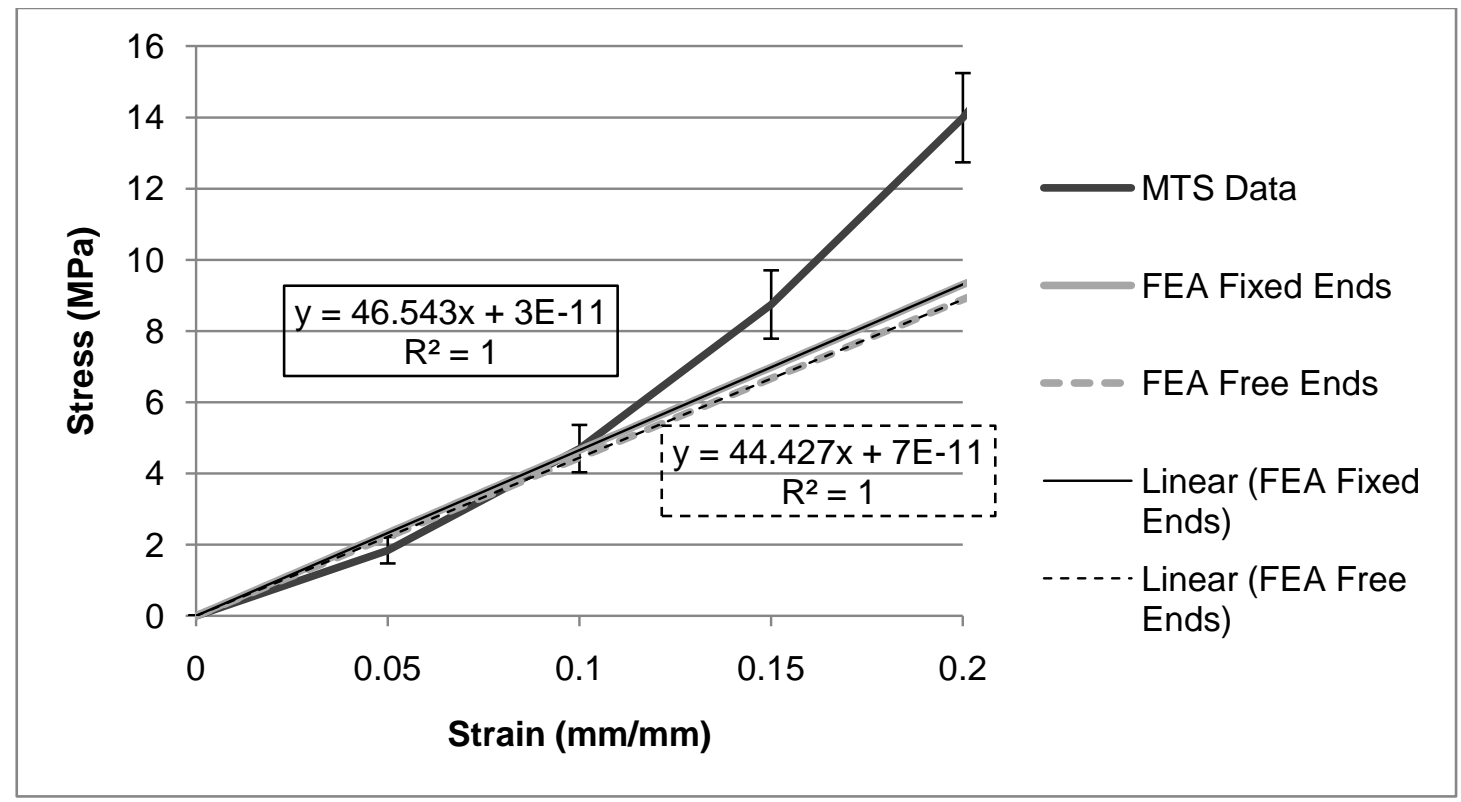

Figure 4.9 Comparison of POC-HA 80-4 Material Models to Experimental Compression Data 


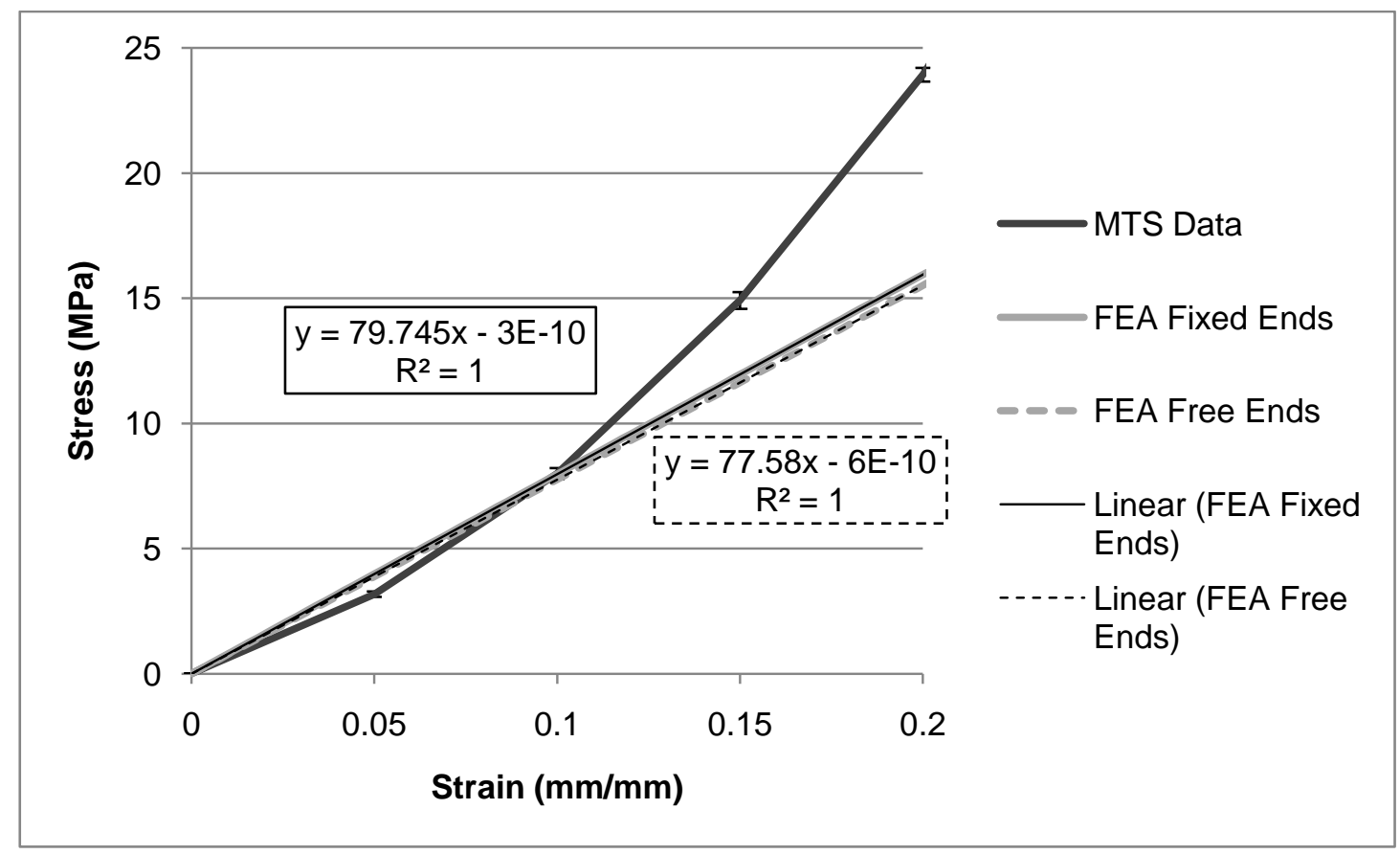

Figure 4.10 Comparison of POC-HA 120-1 Material Models to Experimental Compression Data

The linear elastic model captured the mechanical testing behavior accurately in the 0 to $10 \%$ strain range which is of interest in stent design as shown in Figures 4.9 and 4.10. The difference in the finite element model end conditions had a small effect on the solution which is attributed to the bulging of the specimen due to the smaller aspect ratio. This behavior was observed in the fixed end model as well as in the actual specimen as shown by Figures 4.11 and 4.12. The linear elastic model diverged from the testing data beyond the $10 \%$ strain range as expected since the material model was defined based on the $10 \%$ strain range. To capture the behavior beyond the $10 \%$ strain range, the material model should be changed to a piecewise linear elastic model where a different modulus is specified for the 10 to $20 \%$ strain range. 


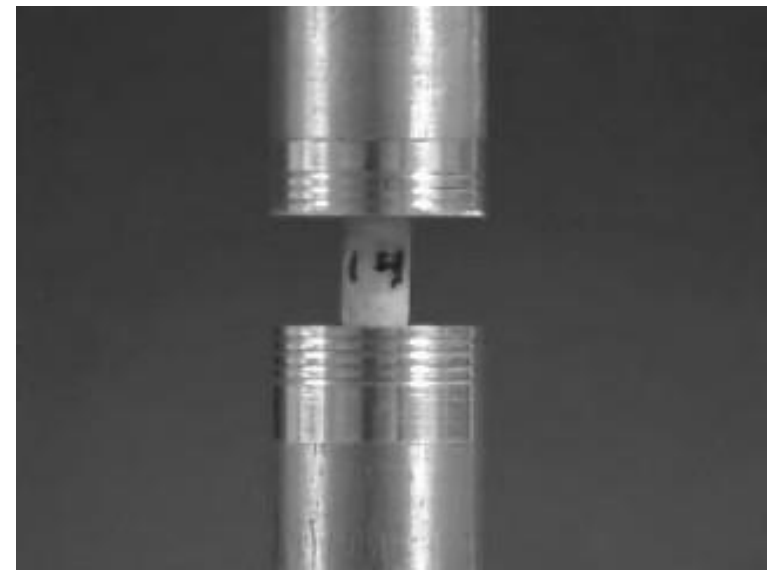

Figure 4.11 POC-HA 120-1 Specimen Compressed to 20\% Strain

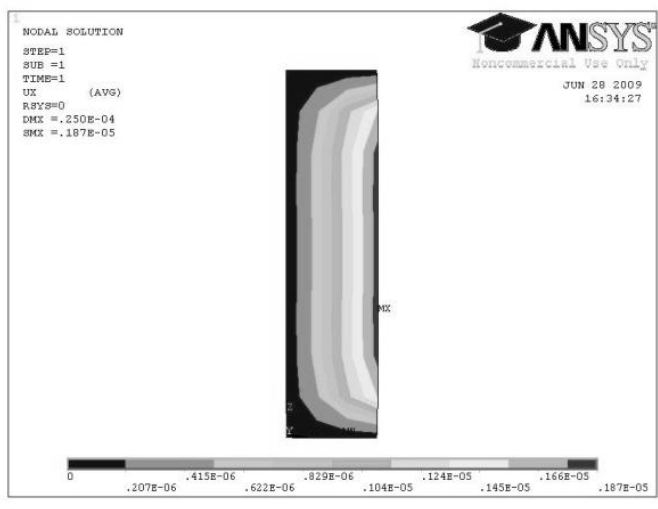

(a)

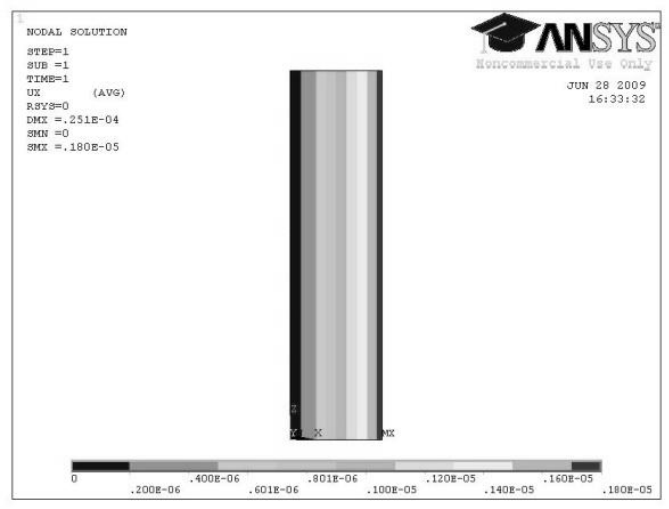

(b)

Figure 4.12 Half Width Lateral Displacements from the POC-HA 120-1

(a) Fixed End and (b) Free End Compression Verification Model

The stress relaxation of the compression verification models are presented in Figure 4.13. For both of the materials the actual specimen relaxed faster than the finite element solution. This might attributed to the defined linear material model in the finite element solution which cannot achieve the same peak stress as the actual specimen. Based on the normalized stress decay in Figure 4.14, the relaxation rates can be compared. The relaxation was nearly $12 \%$ in the finite element solution and around $24 \%$ in the actual test specimen after 10 seconds 
of constant applied strain. The decay is faster in the actual specimen during the first two seconds after which the slopes equalize.

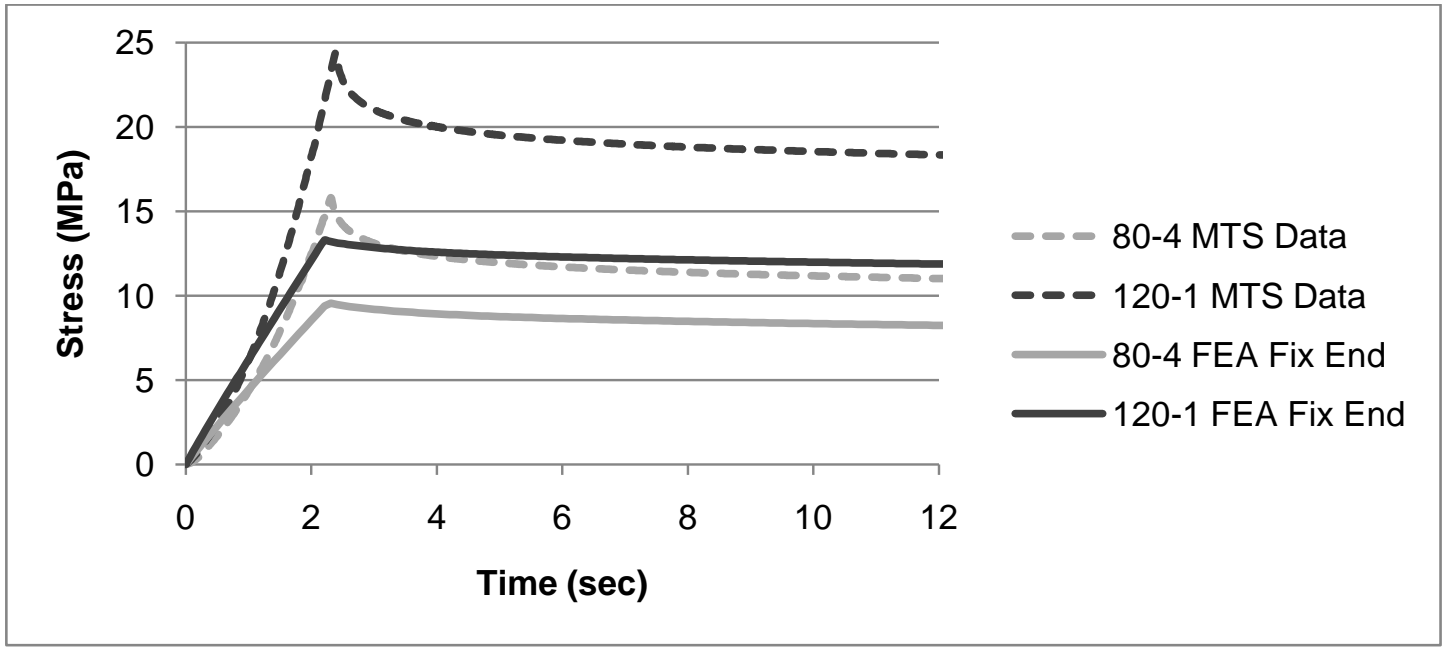

Figure 4.13 Comparison of Stress Relaxation Models with Experimental Compression Data

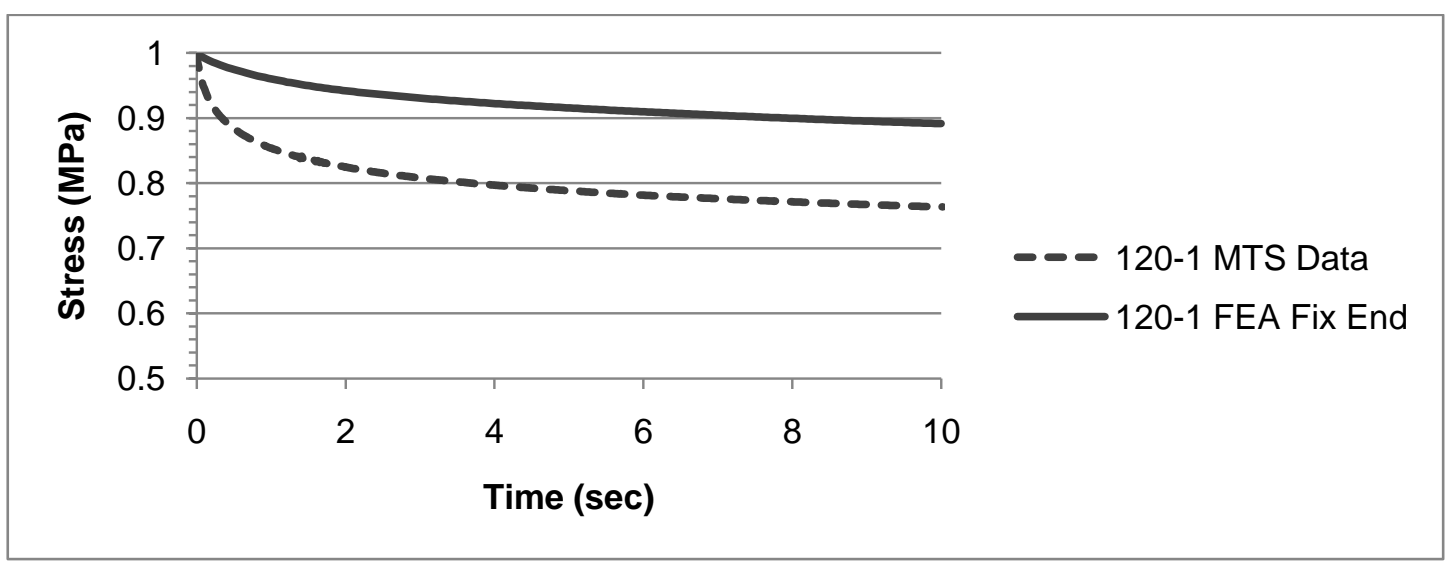

Figure 4.14 The Normalized Stress Relaxation for POC-HA 120-1 in Compression

The stress relaxation was greater in the compression testing than in tensile testing. The same factors affect the finite element results in compression as in tensile testing case, namely the lost relaxation data in the loading phase and the small strain model assumption. 
Since the finite element solution consistently underestimates the stress relaxation a fixed correction factor might be used to correct simulation results.

The material properties used in the compression verification models are summarized in Tables 4.3 and 4.4. EX being the Young's modulus, PRXY being the Poisson's ratio and all of the coefficients are in metric units.

Table 4.3 Material Properties for POCHA 80-4 ANSYS Compression Model (Metric Units)

\begin{tabular}{|c|c|}
\hline \multicolumn{2}{|c|}{ Linear Elastic Isotropic } \\
\hline EX (dry) (Pa) & $4.559 \mathrm{E} 7$ \\
\hline EX (wet) $(\mathrm{Pa})$ & $9.83 \mathrm{E} 6$ \\
\hline PRXY & 0.39 \\
\hline Nonlinear Viscoelastic Prony Shear Response \\
\hline $\mathrm{a} 1$ & $1.7404 \mathrm{E}-18$ \\
\hline $\mathrm{t} 1$ & 0.01 \\
\hline $\mathrm{a} 2$ & 0.084421 \\
\hline $\mathrm{t} 2$ & 0.1 \\
\hline $\mathrm{a} 3$ & 0.12331 \\
\hline $\mathrm{t} 3$ & 1 \\
\hline $\mathrm{a} 4$ & 0.1331 \\
\hline $\mathrm{t} 4$ & 10 \\
\hline
\end{tabular}

Table 4.4 Material Properties for POCHA 120-1 ANSYS Compression Model (Metric Units)

\begin{tabular}{|c|c|}
\hline \multicolumn{2}{|c|}{ Linear Elastic Isotropic } \\
\hline $\mathrm{EX}$ (dry) $(\mathrm{Pa})$ & $7.961 \mathrm{E} 7$ \\
\hline EX (wet) $(\mathrm{Pa})$ & 1.602E7 \\
\hline PRXY & 0.31 \\
\hline \multicolumn{2}{|c|}{ Nonlinear Viscoelastic Prony Shear Response } \\
\hline $\mathrm{a} 1$ & $9.1725 \mathrm{E}-16$ \\
\hline $\mathrm{t} 1$ & 0.01 \\
\hline a2 & 0.096461 \\
\hline $\mathrm{t} 2$ & 0.1 \\
\hline a3 & 0.097466 \\
\hline t3 & 1 \\
\hline a4 & 0.10364 \\
\hline t4 & 10 \\
\hline
\end{tabular}




\subsection{Analysis of Stent Geometry}

An idealized compression analysis was performed on a cylindrical stent strut to evaluate the load bearing capacity of the POC-HA material with strut dimensions found in the general stent literature. It is important to evaluate the load bearing capacity with realistic dimensions before more detailed simulations, optimizations, and pattern designs are performed. Preliminary results can show if the material needs to be improved before efforts are wasted on identifying an optimized design that will not work in a real setting. The load bearing capacity can be achieved for almost any material if the strut thickness is increased. However, one must keep in mind that for delivery the stent must be crimped to the catheter for delivery which imposes a constraint on the allowable dimensions; for $3 \mathrm{~mm}$ diameter stents the currently used catheters have an inner diameter of around $1.5 \mathrm{~mm}$.

In the current metallic drug eluting stents the strut thicknesses are around 100 microns or below. The strut thickness in the PLLA Igaki-Tamai stent was 170 microns and it covered $24 \%$ of the vessel area [Tamai, 2000]. The PLLA based BVS stent by Abbott has a nominal diameter of $3.0 \mathrm{~mm}$ and strut thickness of 150 microns [Ormiston, 2008]. A PLLA/P4HB blend material based stent by Grabow et. al. which has a slotted tube design with dimensions of $6 \mathrm{~mm}$ nominal diameter and a 300 micron thickness was used as a basis in this analysis [Grabow, 2007]. The stents with the dimensions mentioned had collapse pressures comparable to metallic stents and were deliverable with conventional methods. The stent pattern used in the analysis is shown in Figure 4.15. The model shown has a $3 \mathrm{~mm}$ outer diameter, 200 micron thickness, and $20 \%$ artery wall coverage (ratio of width of cylindrical strut to width of open area in repeated cell). 


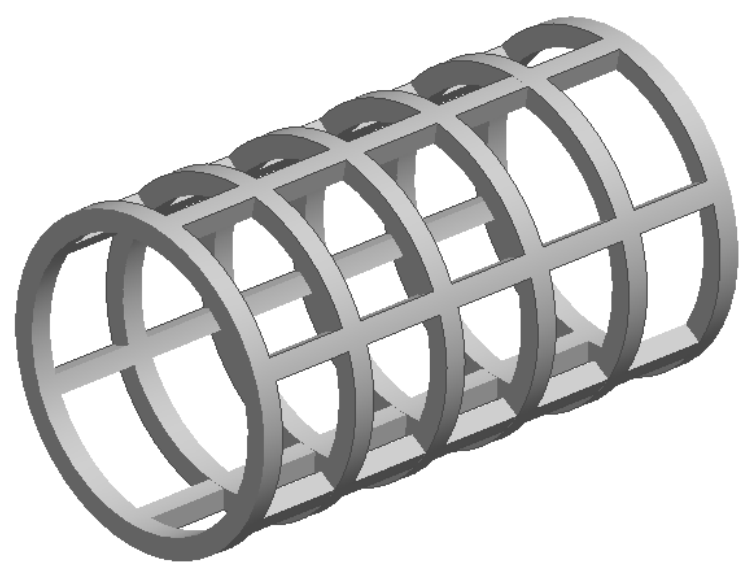

Figure 4.15 Slotted Tube Stent Design

A quarter of a cylindrical load bearing strut was selected for analysis. It was assumed that the artery wall perfectly transmits the entire load from the open areas to the strut creating an increased equivalent pressure. This analysis has none of the structural nonlinearities present in the physical loading case such as buckling and non-uniform pressure distributions. This idealized hydrostatic loading case provides the absolute maximum structural support that can be achieved with the current material and realistic stent dimensions.

The material model used was for POC-HA 120-1 with compressive material properties for both the dry and wet conditions. The model in Figure 4.16 shows the applied loads to the strut and boundary conditions used in the analysis. The boundary conditions were defined to be no axial Z-displacement at one side to keep the strut straight and symmetry boundary conditions at the cut planes (no X-displacement on YZ plane and Y-displacement on XZ plane). An equivalent pressure of 1 bar was applied to the outer surface of the strut. For example in the $20 \%$ artery wall coverage case the equivalent pressure is 5 bar. The model was solved in ANSYS using small displacement transient analysis with a ramped pressure loading from zero to applied pressure in 1 second after which a load step of constant pressure was held for 1 second to incorporate the stress relaxation effect. 


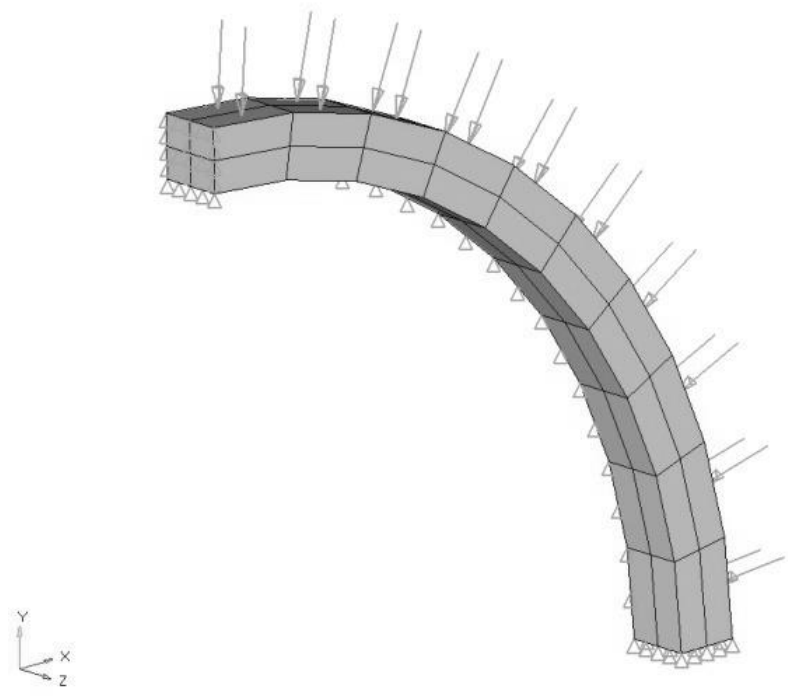

Figure 4.16 Quarter Strut Model with Equal Pressure Loading

The results of the analysis are presented in Tables 4.5 and 4.6. The recoil was calculated as the change in inner radius after relaxation divided by the original inner radius. The current POC-HA material could achieve recoil levels comparable to metallic stents in the dry material condition [Lanzer, 2007]. When the material is subject to body conditions, wet and 37 ${ }^{\circ} \mathrm{C}$, the structural support is lost even at this much idealized loading condition.

In real artery loading which is not perfectly distributed, the Igaki-Tamai stent was measured to have $22 \pm 7 \%$ acute recoil when the vascular stenosis was reduced from $64 \%$ to 12\% [Tamai, 2000]. For the POC-HA material to provide sufficient scaffolding in real artery loading it must perform better in finite element simulations than the stents in real loading to account for all the loading imperfections and nonlinearities which are not considered in the analysis. In summary, the POC-HA material in its current formulation does not have sufficient mechanical strength to be employed for a coronary stent scaffolding function. 
Table 4.5 Stent Recoil for POC-HA 120-1 dry at RT

\begin{tabular}{|c|c|c|c|}
\hline & \multicolumn{3}{|c|}{ Strut Thickness (microns) } \\
Artery Coverage & 150 & 200 & 300 \\
\hline $20 \%$ & $7.9 \%$ & $6.0 \%$ & $4.2 \%$ \\
\hline $30 \%$ & $5.2 \%$ & $4.0 \%$ & $2.8 \%$ \\
\hline
\end{tabular}

Table 4.6 Stent Recoil for POC-HA 120-1 at Body Conditions

\begin{tabular}{|c|c|c|c|}
\hline & \multicolumn{3}{|c|}{ Strut Thickness (microns) } \\
Artery Coverage & 150 & 200 & 300 \\
\hline $20 \%$ & $39.3 \%$ & $30.1 \%$ & $20.8 \%$ \\
\hline $30 \%$ & $26.2 \%$ & $20.0 \%$ & $13.8 \%$ \\
\hline
\end{tabular}




\section{CHAPTER 5}

\section{CONCLUSIONS AND RECOMMENDATIONS}

\subsection{Conclusions}

The important mechanical properties for the POC-HA polymeric composite material were characterized by tensile and compressive experiments utilizing established test protocols and commercially available testing equipment. The material properties obtained from the compression testing should be used in the design of a self-expanding polymeric stent since the loads in the body due to the artery walls are always compressive, and the repeatability in the compression test data were higher than in tensile testing.

There were multiple factors which affected the mechanical properties of the POC-HA material. The stiffness and load bearing capacity of the material were increased with increasing crosslinking times and temperatures. This increase was achieved with just a small decrease in failure strain. The material also behaved stiffer under compression than tensile loading.

The analyzed loading cases which had a major degrading effect on the material properties were cyclic loading and wet material condition. Repeated loading reduced the material stiffness by $25 \%$ after three initial cycles and then remained fairly constant up to ten cycles. There was also a $40 \%$ loss in load bearing capacity of the material at $10 \%$ strain after five cycles.

The POC-HA material was capable of absorbing a high concentration (nearly 13 to $15 \%$ by weight) of PBS solution. The swelling behavior was lesser in the material with higher crosslinking. The wet condition decreased the stiffness and load bearing capacity of both POCHA materials by nearly $80 \%$. 
The mechanical behavior of the POC-HA polymeric composite material was simulated in a commercial finite element analysis code with readily available element and material model formulations. The presented finite element analysis stress-strain results correlate well in the 0 to10\% strain range which is of interest in stent design. The viscoelastic behavior could not be captured as accurately with the models. For example in the compression POC-HA 120-1 model the stress relaxation was only $12 \%$ while in the actual testing the relaxation was measured to be $24 \%$ after 10 seconds of constant applied strain. These results were obtained with a simple linear-elastic with small strain viscoelasticity material model.

An idealized compression loading analysis with the developed material model was performed on a load bearing stent strut with dimensions that are currently used in biodegradable coronary stents. Based on this analysis the POC-HA material at body conditions cannot provide adequate scaffolding with dimensions that are comparable to current biodegradable stents and realistic in stent application.

The femtosecond laser fabrication method can be utilized for stent fabrication. The femtosecond laser machining resulted in high quality surfaces with no damage to surrounding material.

The presented material properties and simulation models can be used to design POCHA based biodegradable devices. Also, the presented material characterization and finite element design methods can be utilized with future polymeric materials to design cardiovascular stents.

\section{$\underline{5.2 \text { Recommendations }}$}

It is recommended that due to the low stiffness characteristic at realistic application conditions the suitability of POC-HA material for another stent application such as peripheral or urology be evaluated as for these types of stents the load bearing requirements might be less than for the coronary application. Also, the POC-HA material should be investigated for stent 
coating and for drug delivery applications. The property of liquid absorption may be beneficial in drug delivery devices with controlled release rates.

The benefits of the femtosecond laser micro-machining method should be further evaluated. One of such experiments is to evaluate the effect of femtosecond laser machining on hemocompatibility and endothelial cell growth on laser machined surfaces. One of the research focuses in stent technology is to find what effect the material surface morphology has on the healing process of the artery wall.

One of the mechanical behaviors not investigated in this study was the effect of material degradation on the mechanical properties. The wet degradation mechanism of the POC-HA material should be characterized to understand when and what causes the material to lose its mechanical strength and if the degradation occurs on the surface or in the bulk of the material. Also, the thermo-mechanical properties of the material should be evaluated to determine how the addition of $\mathrm{HA}$ changes the glass transition temperature from pure POC.

The actual collapse pressures for the patterns presented in the analysis should be evaluated. The fabricated stents should be loaded in the developed pressure testing bench. With an applied external pressure the actual instability of the pattern and the material can be evaluated. This would provide the correlation between actual collapse and finite element collapse pressures which can be used to evaluate the ability of finite element analysis to predict stent recoil and collapse. For example the finite element solution may consistently overestimate the actual collapse pressure by a certain fraction and could be used as a correction factor in the design. Also, the measured stent dimensions at collapse could be used as a secondary failure criterion in analyzing the performance of a stent design based on simulations results. This correlation would provide a guideline for achieving a desired actual collapse pressure for a stent designed with finite element analysis.

Finally the hemocompatibility of POC-HA material must be investigated. This is one of the major requirements for stent design. Even if the stent pattern and the material can provide 
the required scaffolding function and it can be delivered, it cannot be utilized in patient trials unless the hemocompatibility and biocompatibility of the material is proven in animal testing. 
APPENDIX A

TENSILE TESTING DATA 


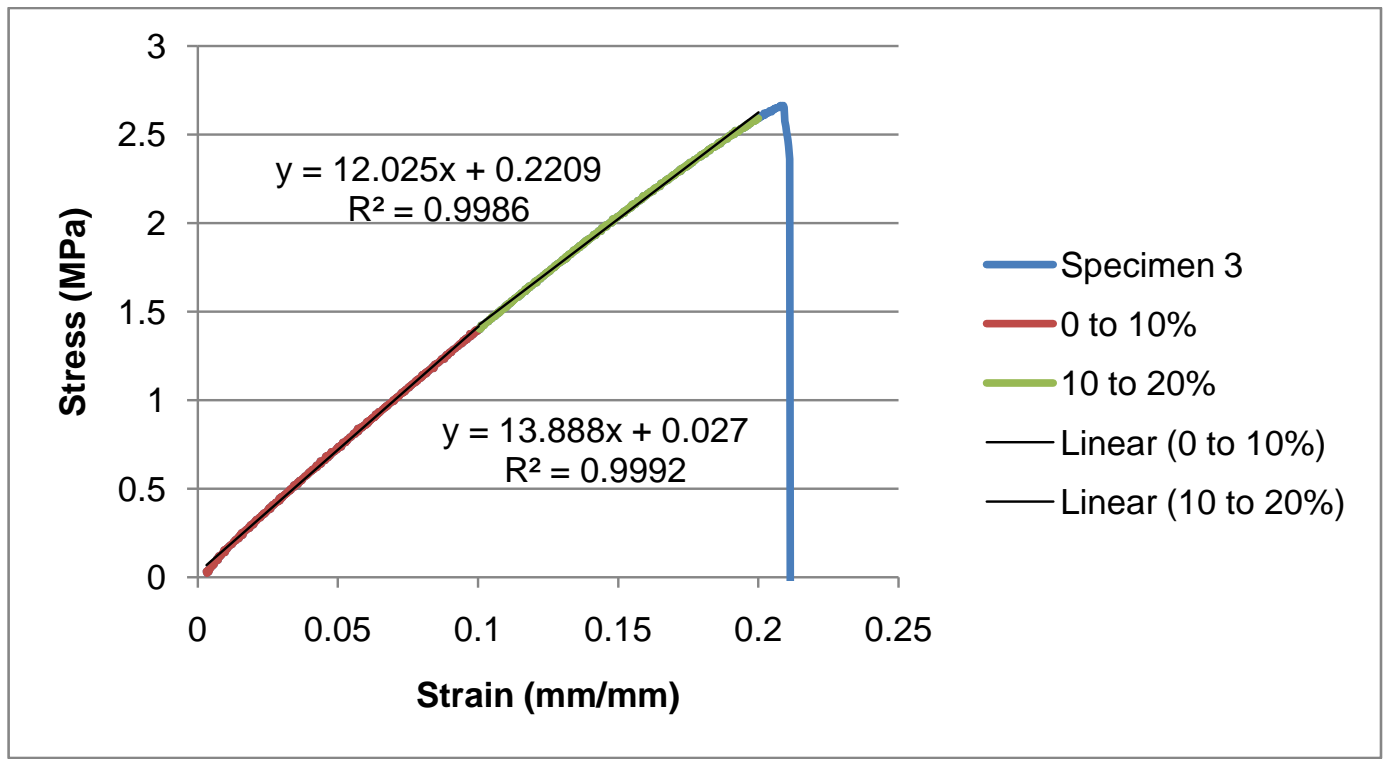

Figure A.1 Stress-Strain Behavior of POC-HA 80-4 Specimen 3 (50 mm/min)

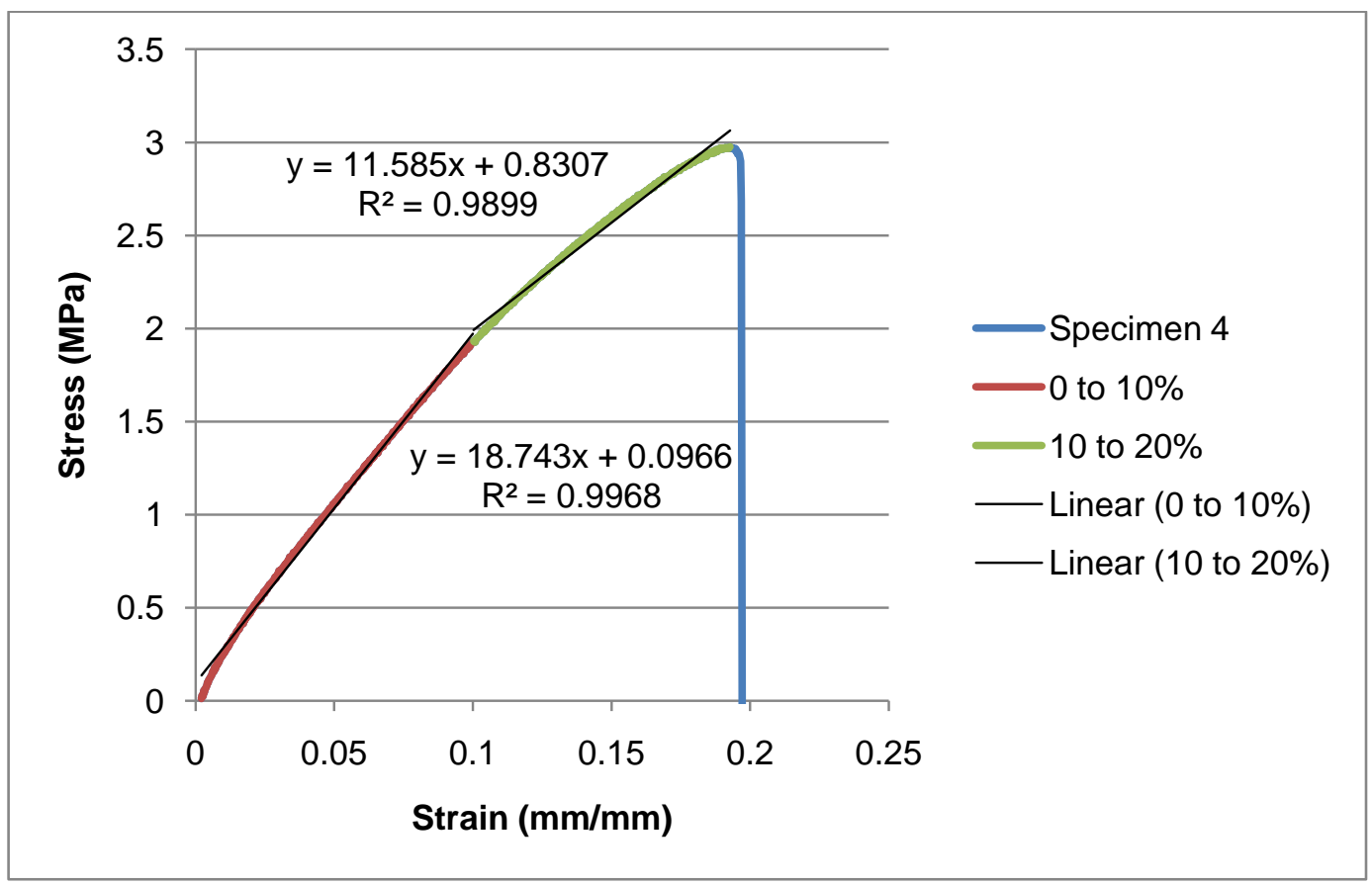

Figure A.2 Stress-Strain Behavior of POC-HA 80-4 Specimen 4 (50 mm/min) 


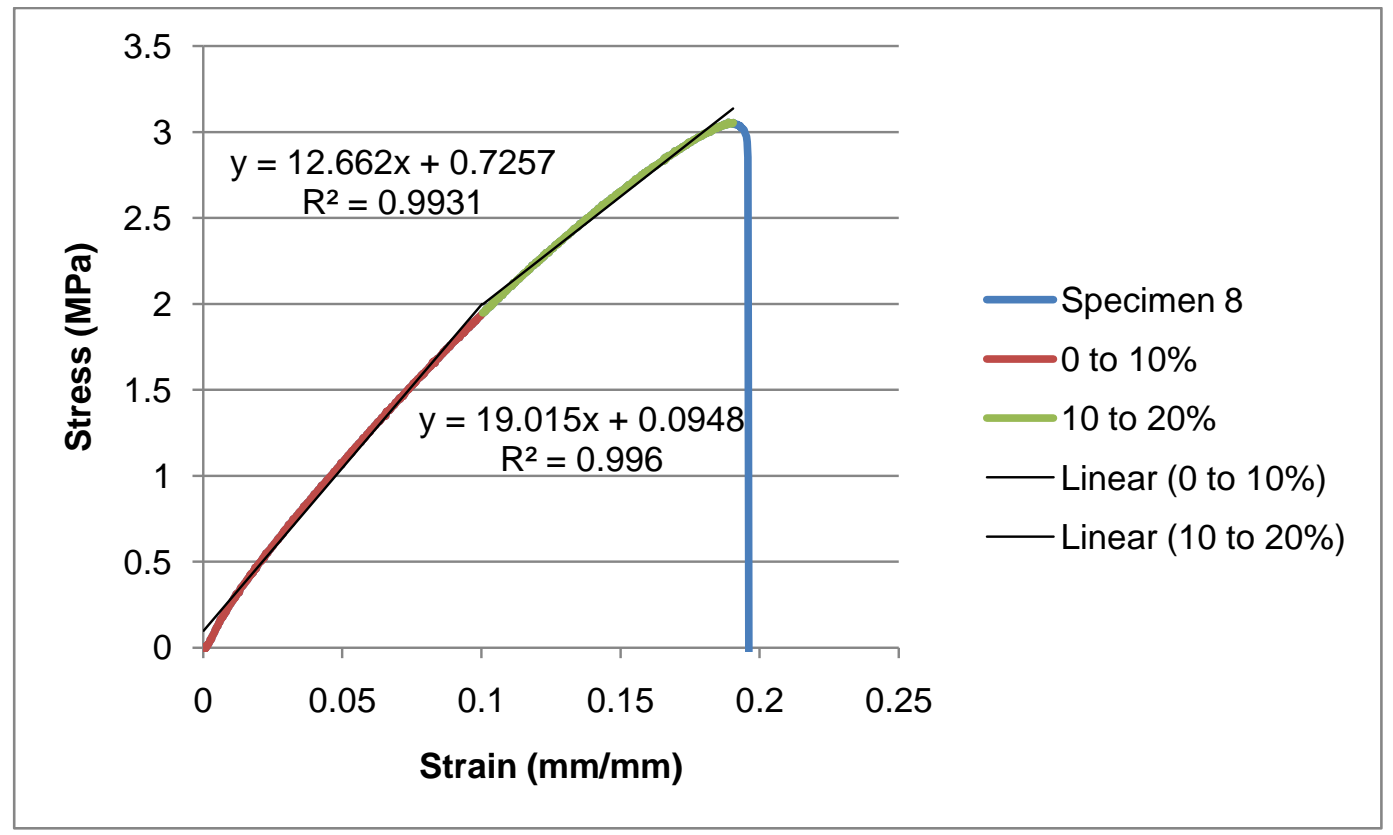

Figure A.3 Stress-Strain Behavior of POC-HA 80-4 Specimen 8 (50 mm/min)

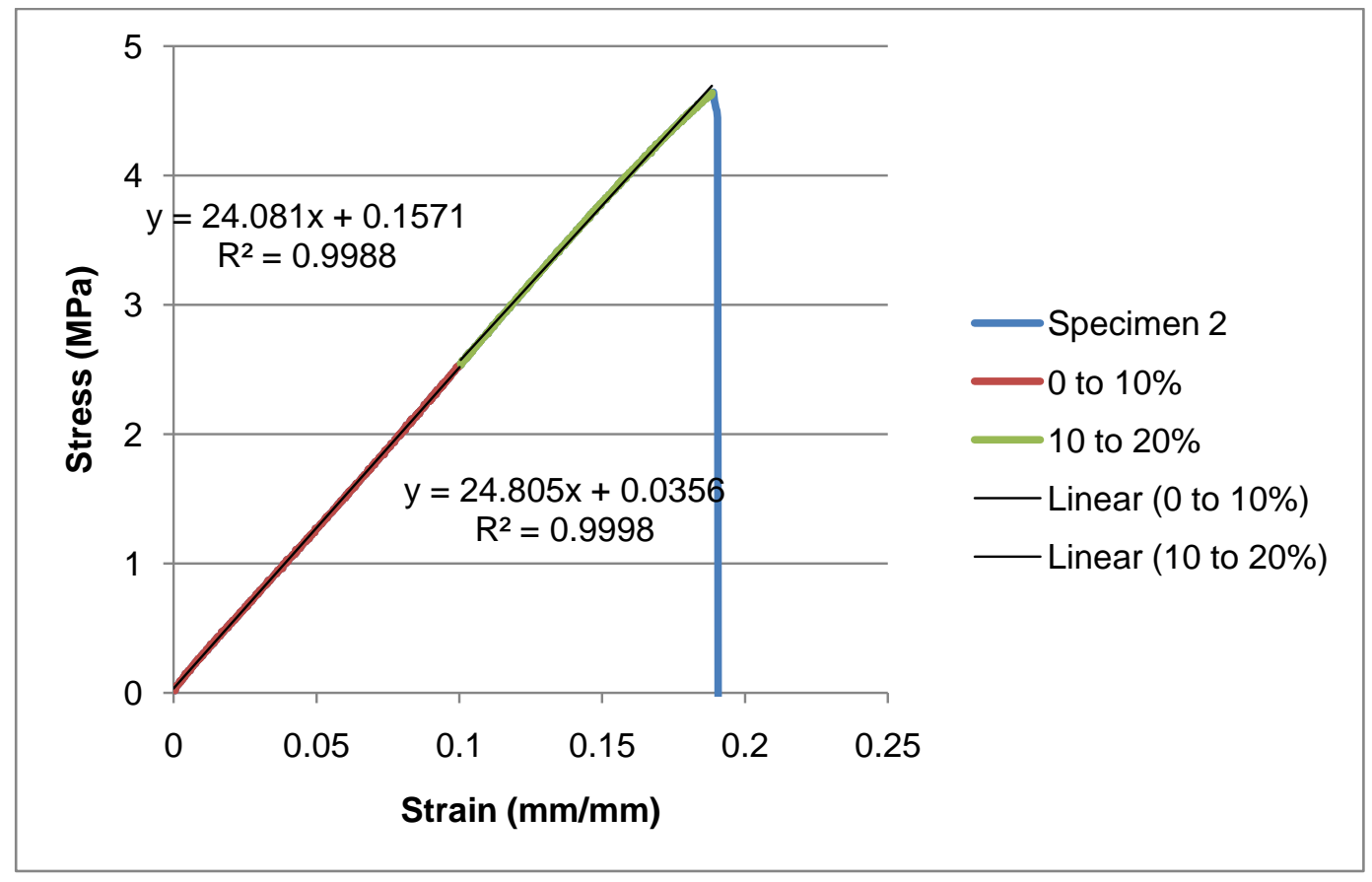

Figure A.4 Stress-Strain Behavior of POC-HA 120-1 Specimen 2 (50 mm/min) 


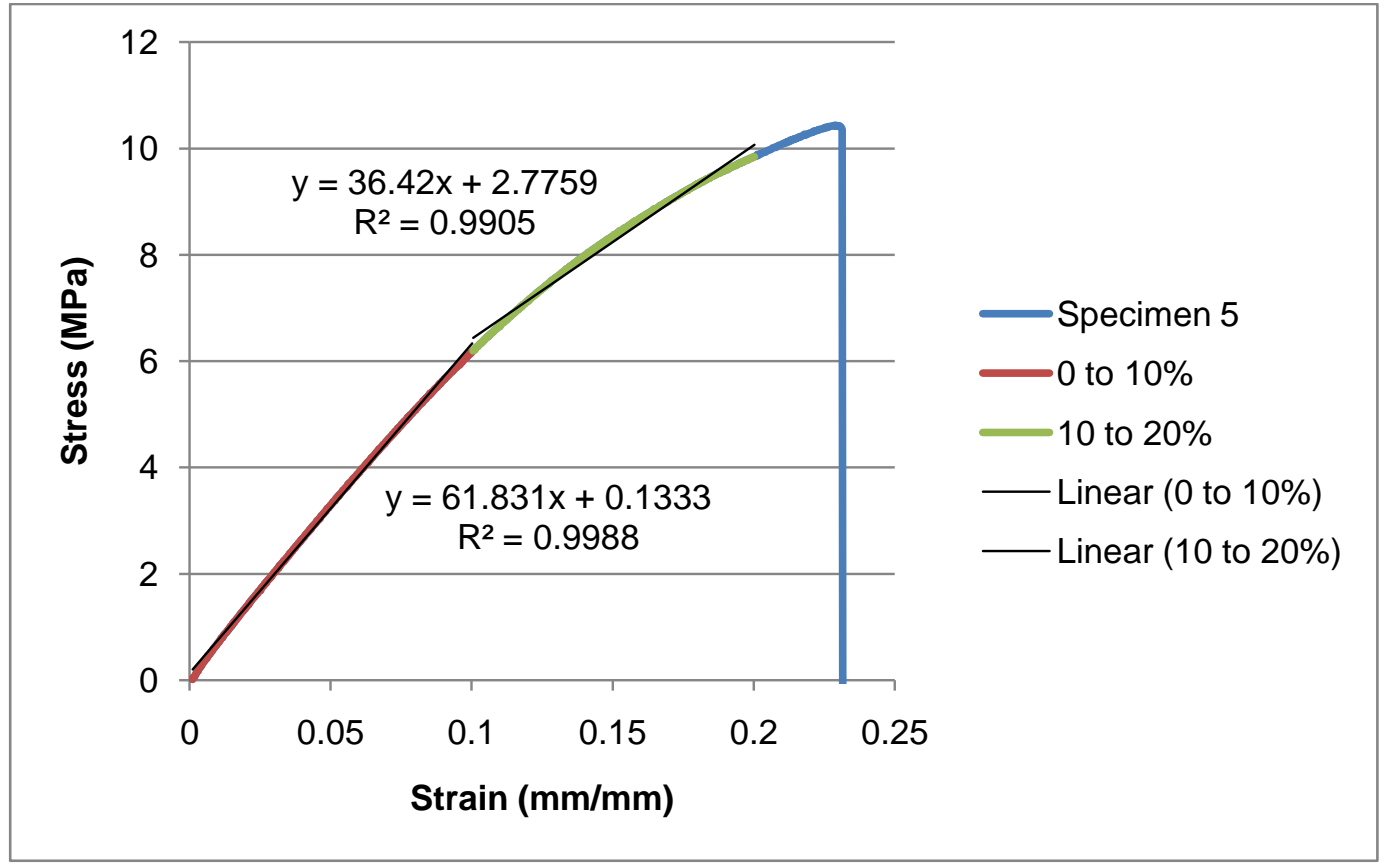

Figure A.5 Stress-Strain Behavior of POC-HA 120-1 Specimen 5 (50 mm/min)

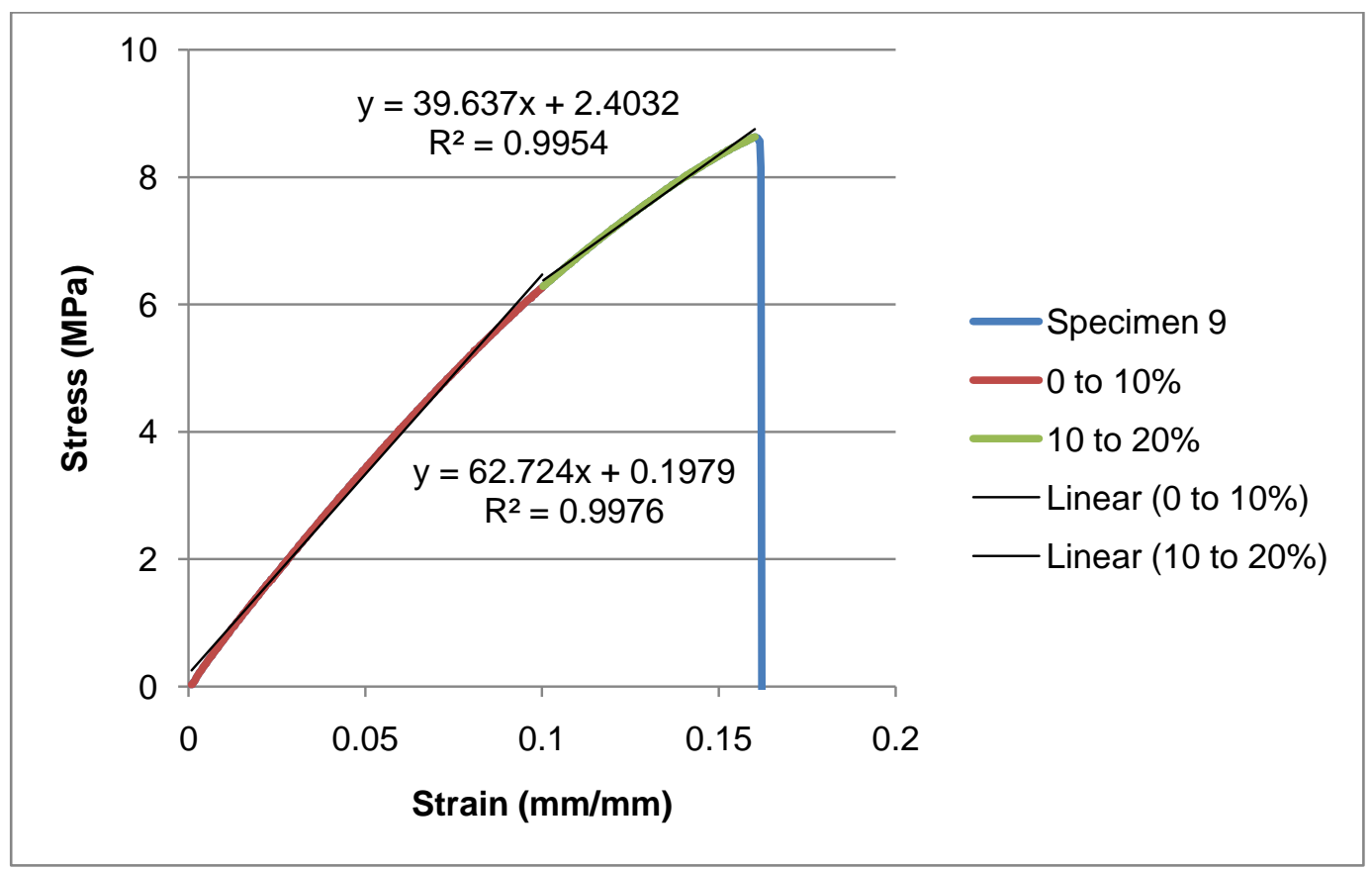

Figure A.6 Stress-Strain Behavior of POC-HA 120-1 Specimen 9 (50 mm/min) 


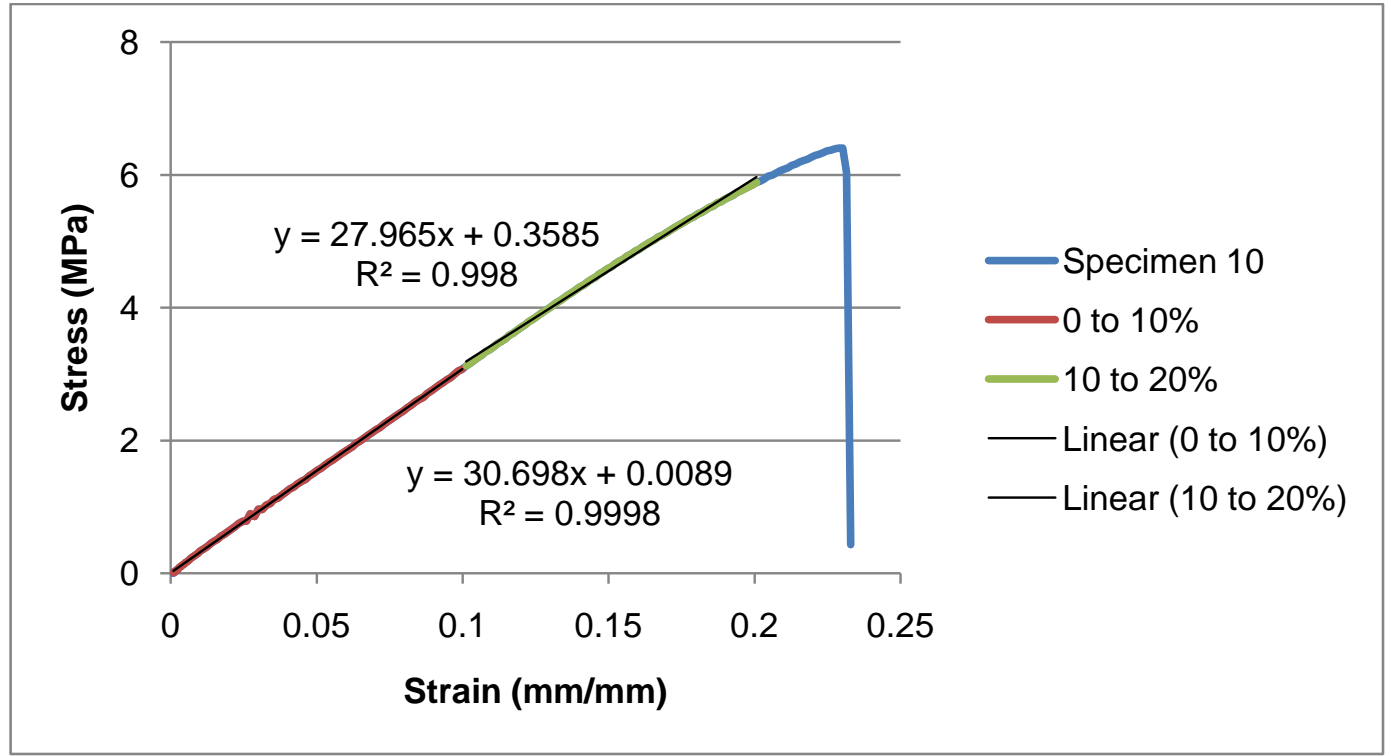

Figure A.7 Stress-Strain Behavior of POC-HA 120-1 Specimen 10 (500 mm/min)

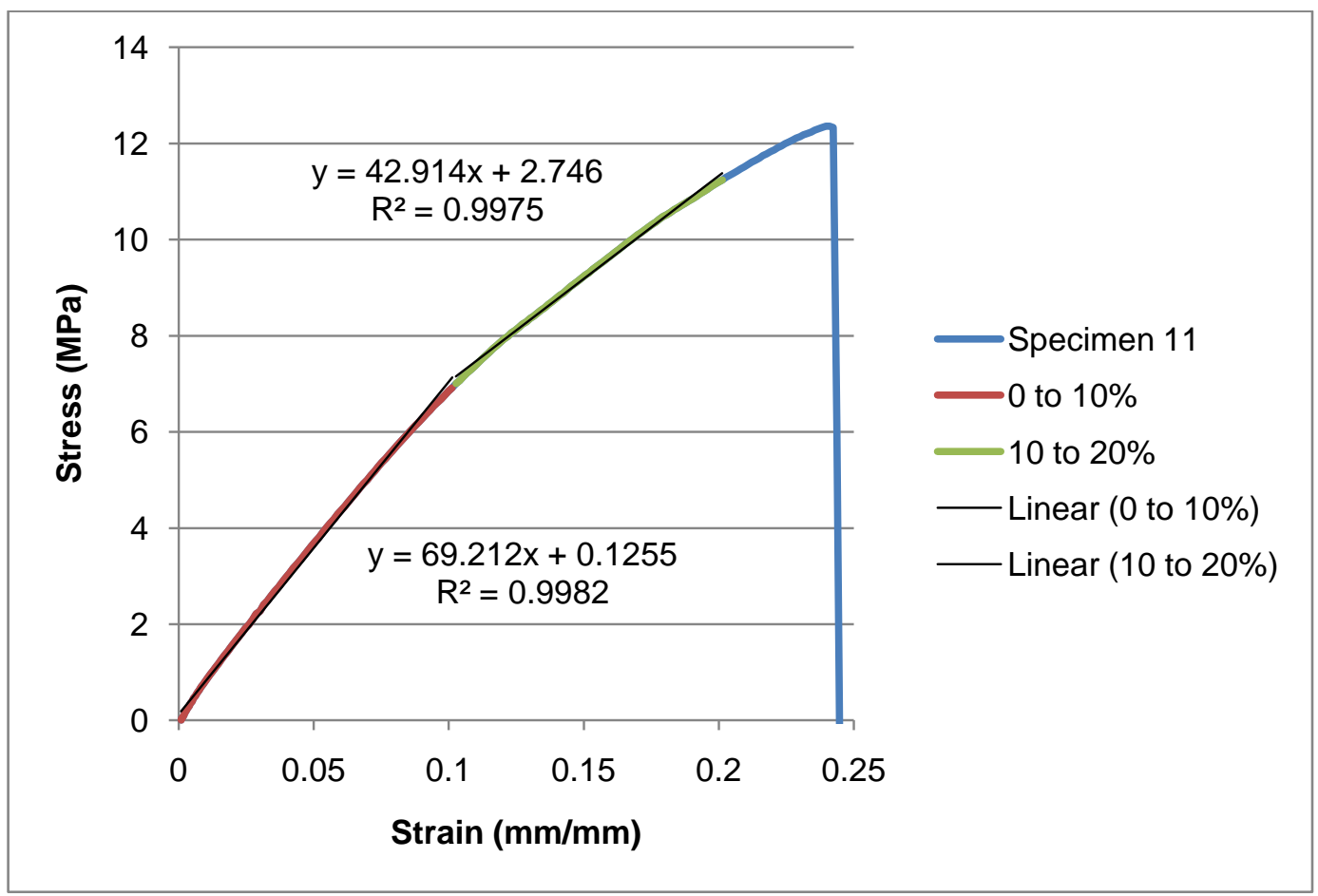

Figure A.8 Stress-Strain Behavior of POC-HA 120-1 Specimen 11 (500 mm/min) 


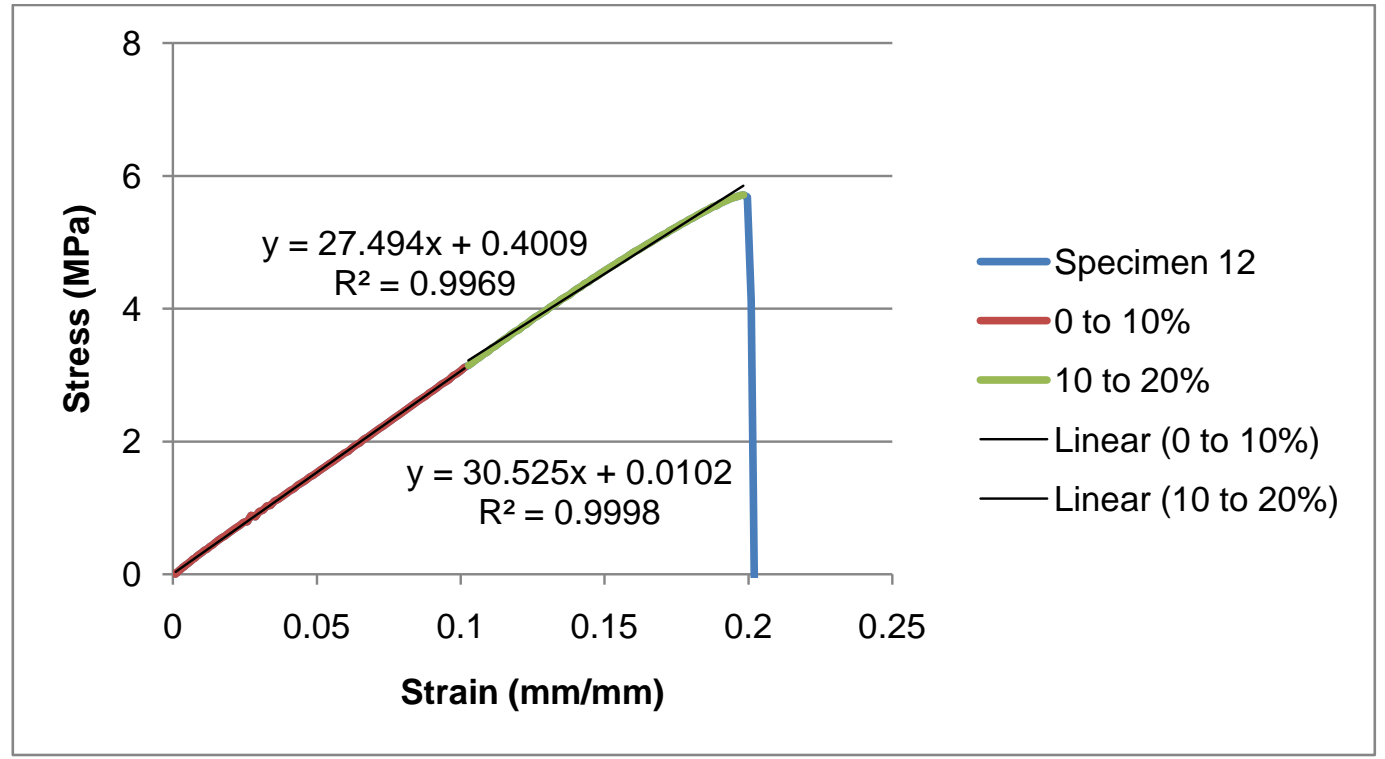

Figure A.9 Stress-Strain Behavior of POC-HA 120-1 Specimen 12 (500 mm/min)

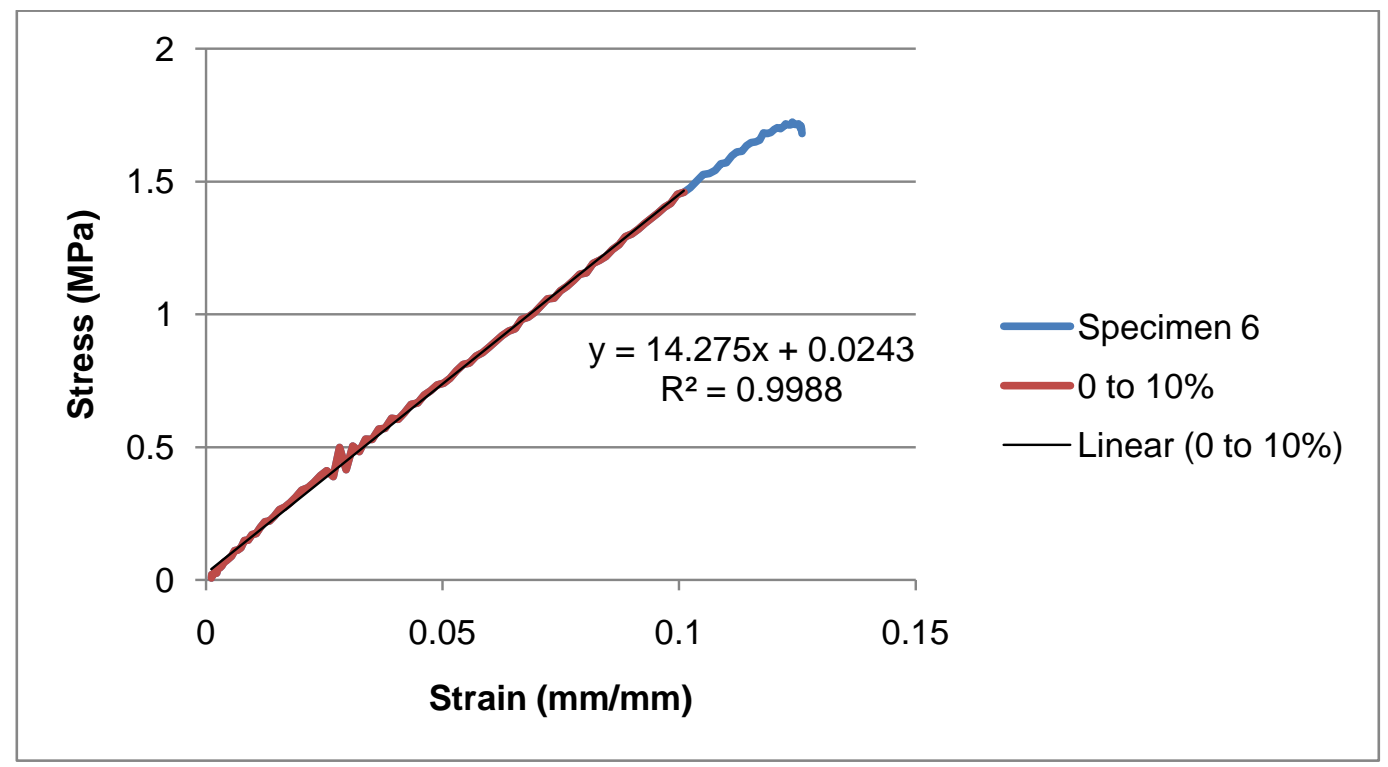

Figure A.10 Stress-Strain Behavior of POC-HA 80-4 Specimen $6(500$ mm/min) 


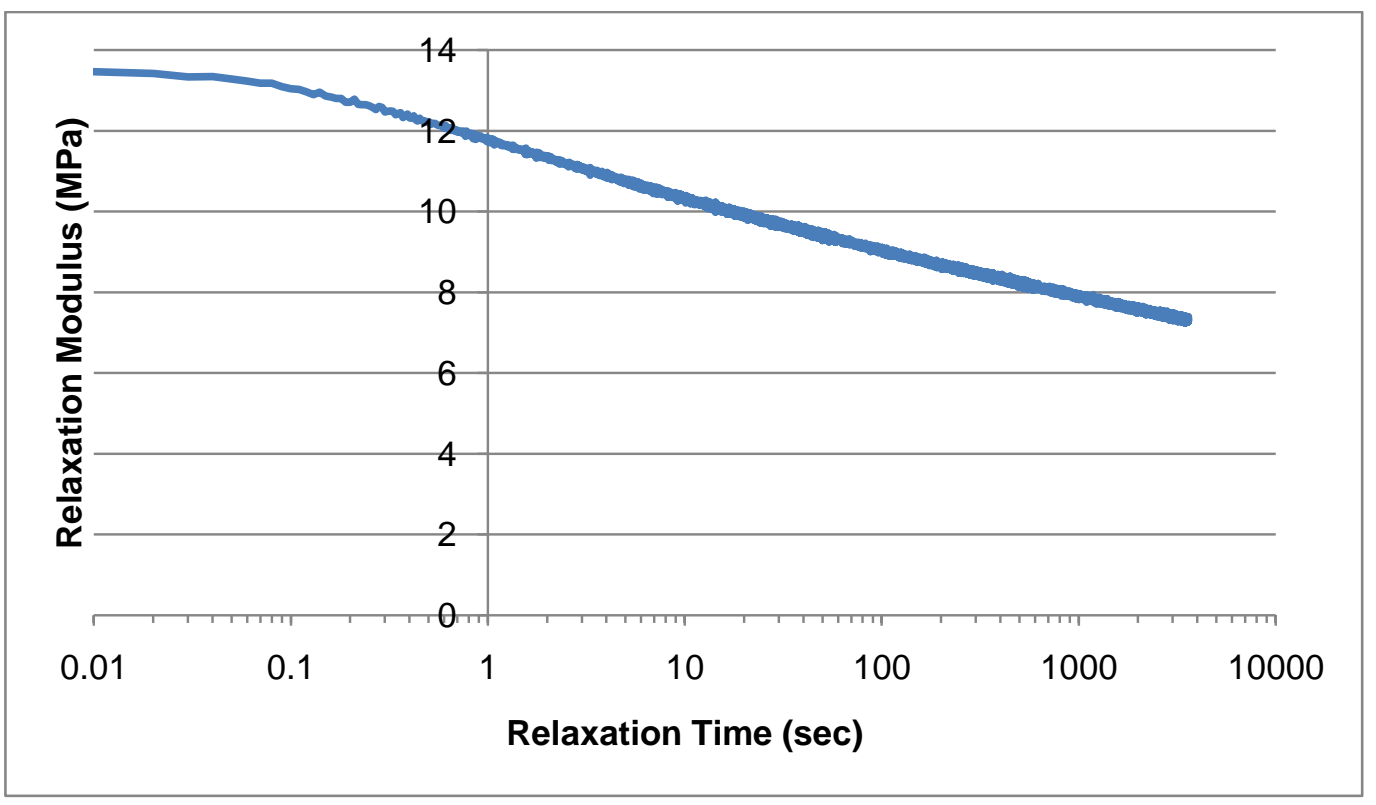

Figure A.11 Relaxation Modulus of POC-HA 80-4 Specimen 6

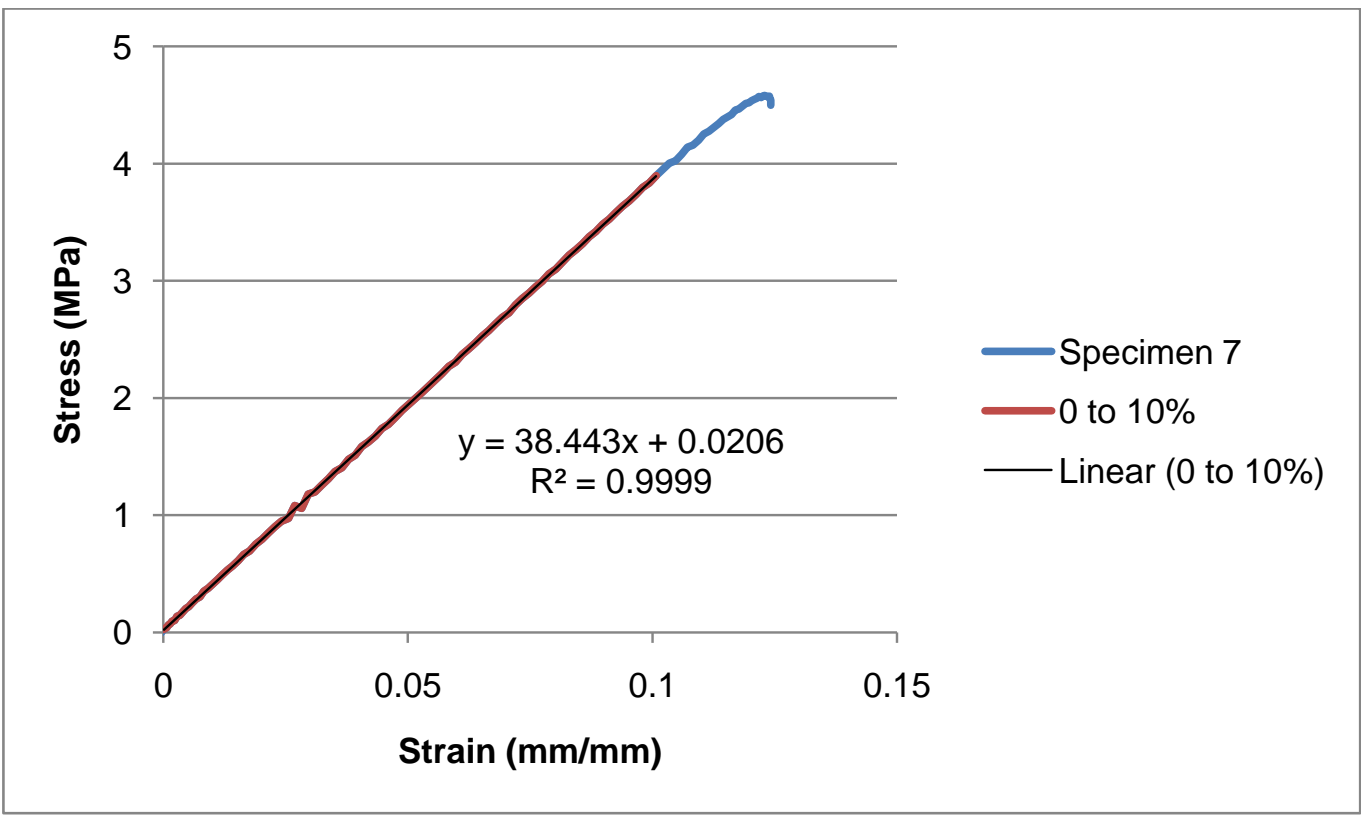

Figure A.12 Stress-Strain Behavior of POC-HA 120-1 Specimen 7 (500 mm/min) 


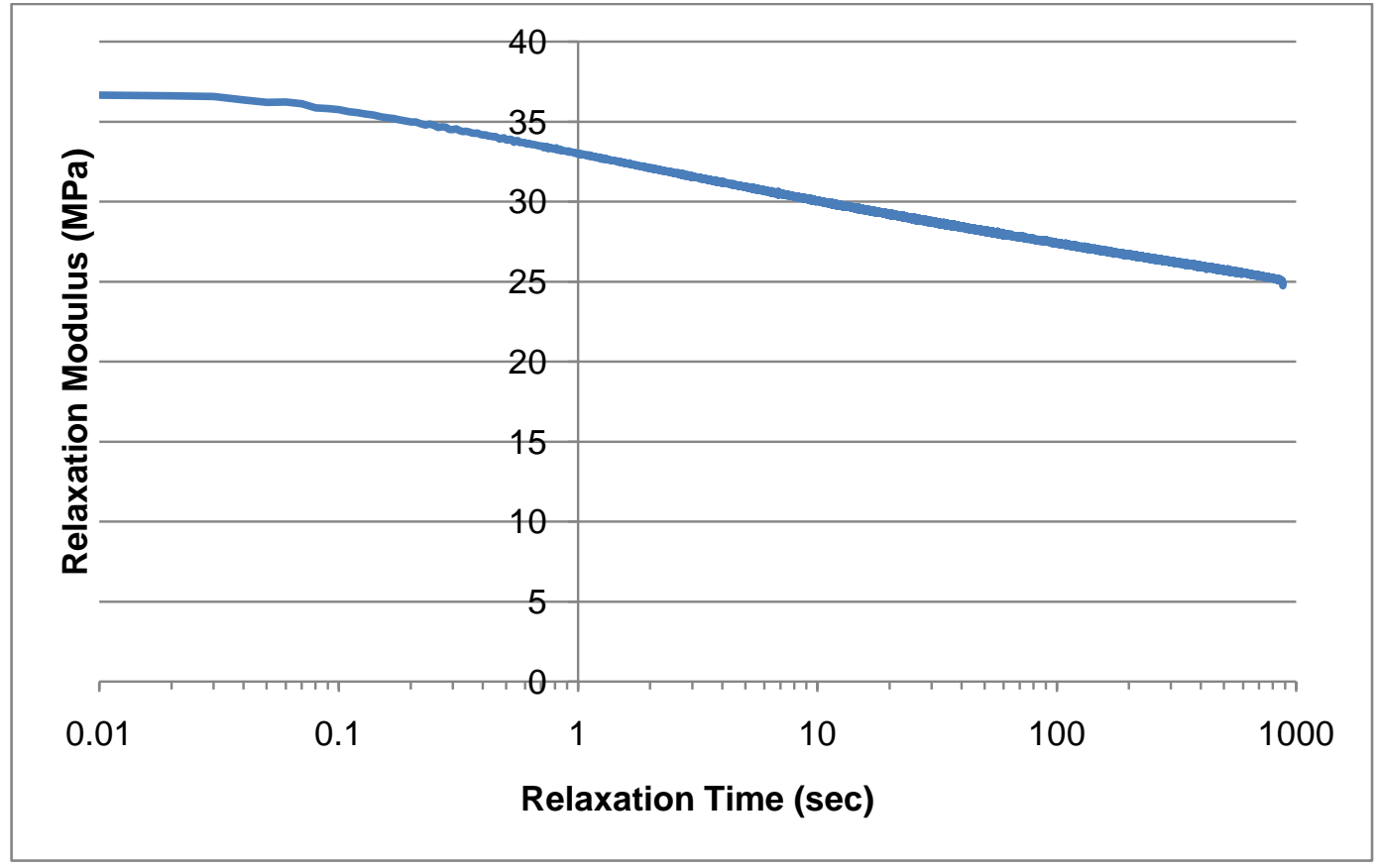

Figure A.13 Relaxation Modulus of POC-HA 120-1 Specimen 7 
APPENDIX B

COMPRESSION TESTING DATA 


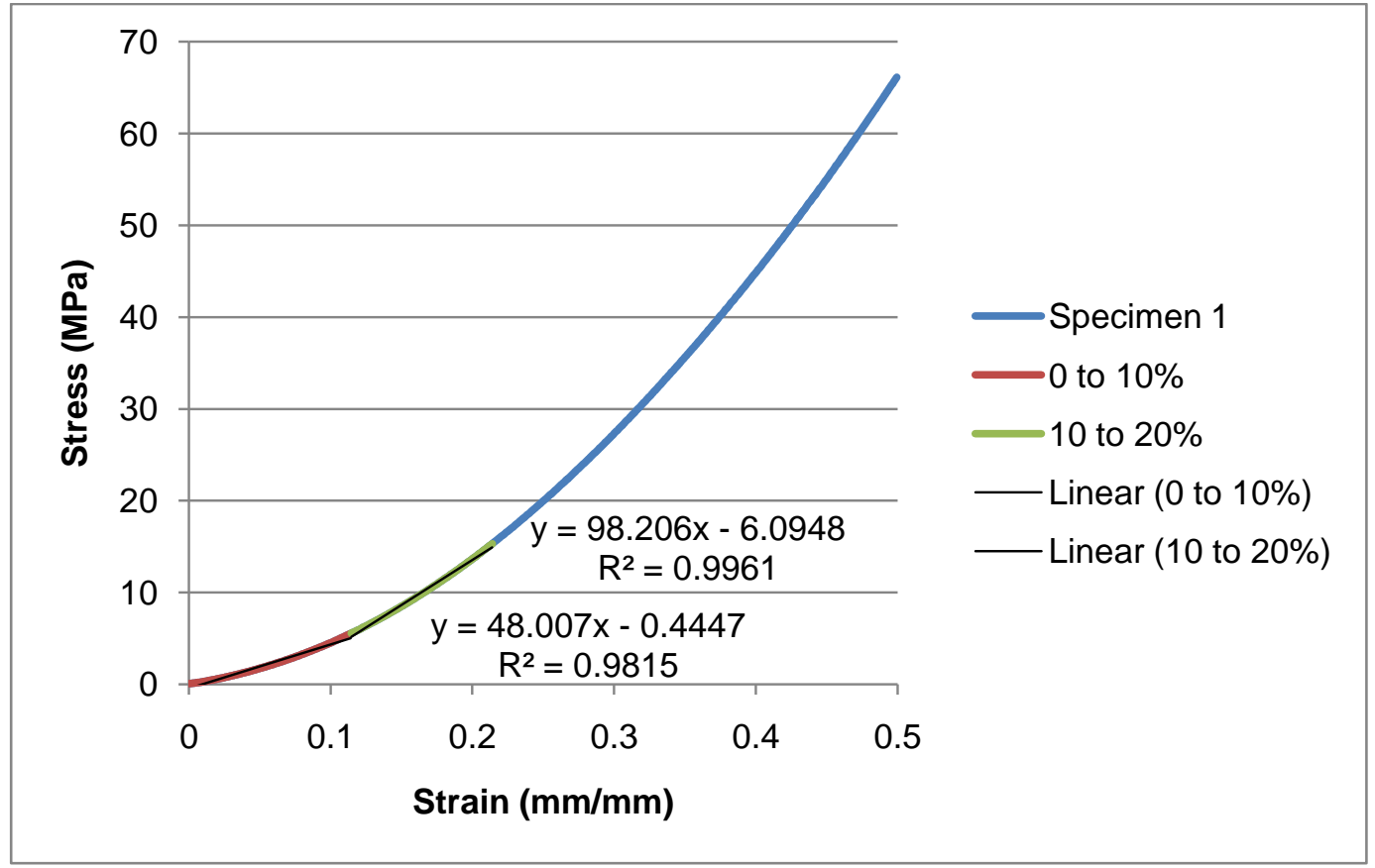

Figure B.1 Stress-Strain Behavior of dry POC-HA 80-4 Specimen 1

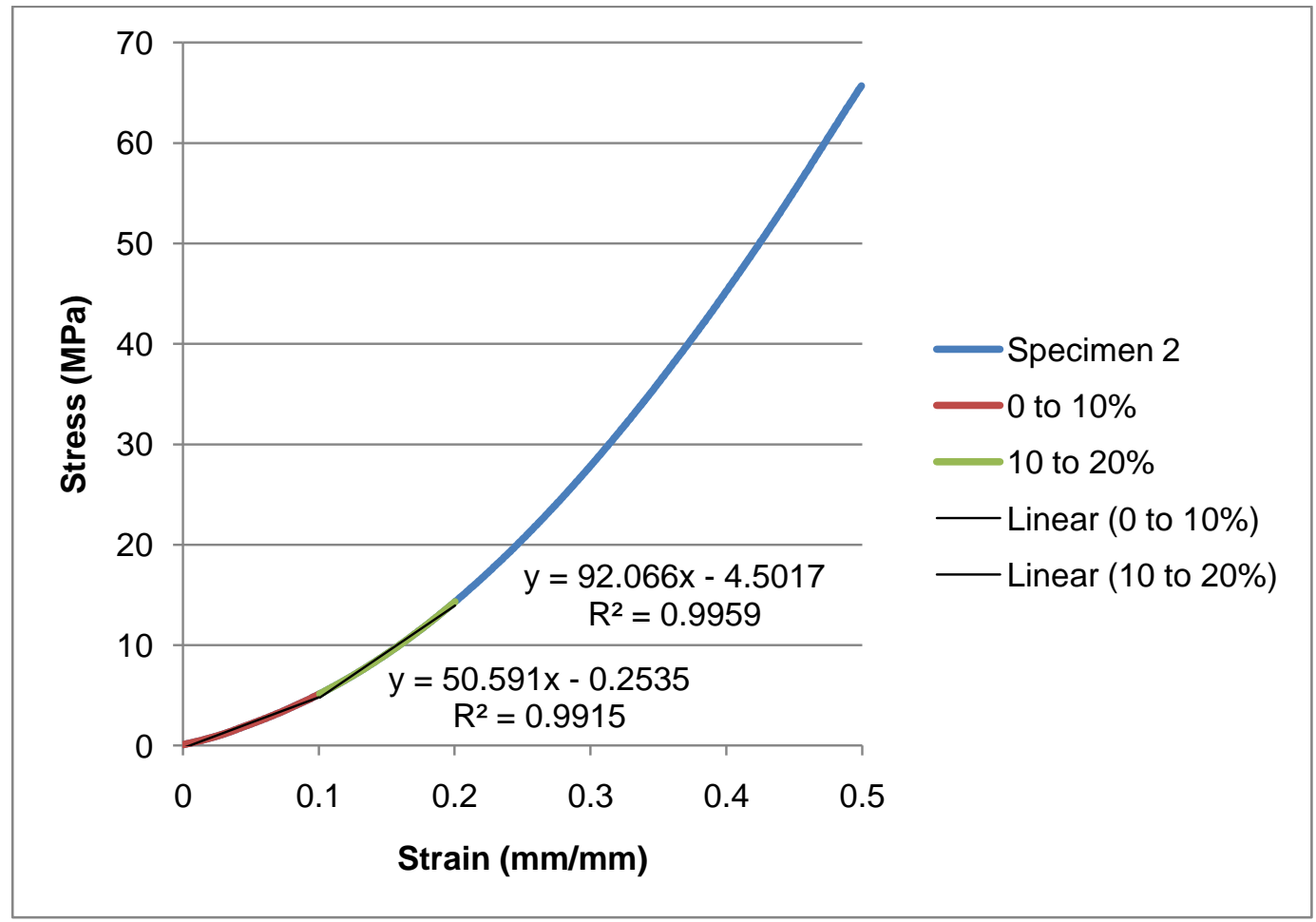

Figure B.2 Stress-Strain Behavior of dry POC-HA 80-4 Specimen 2 


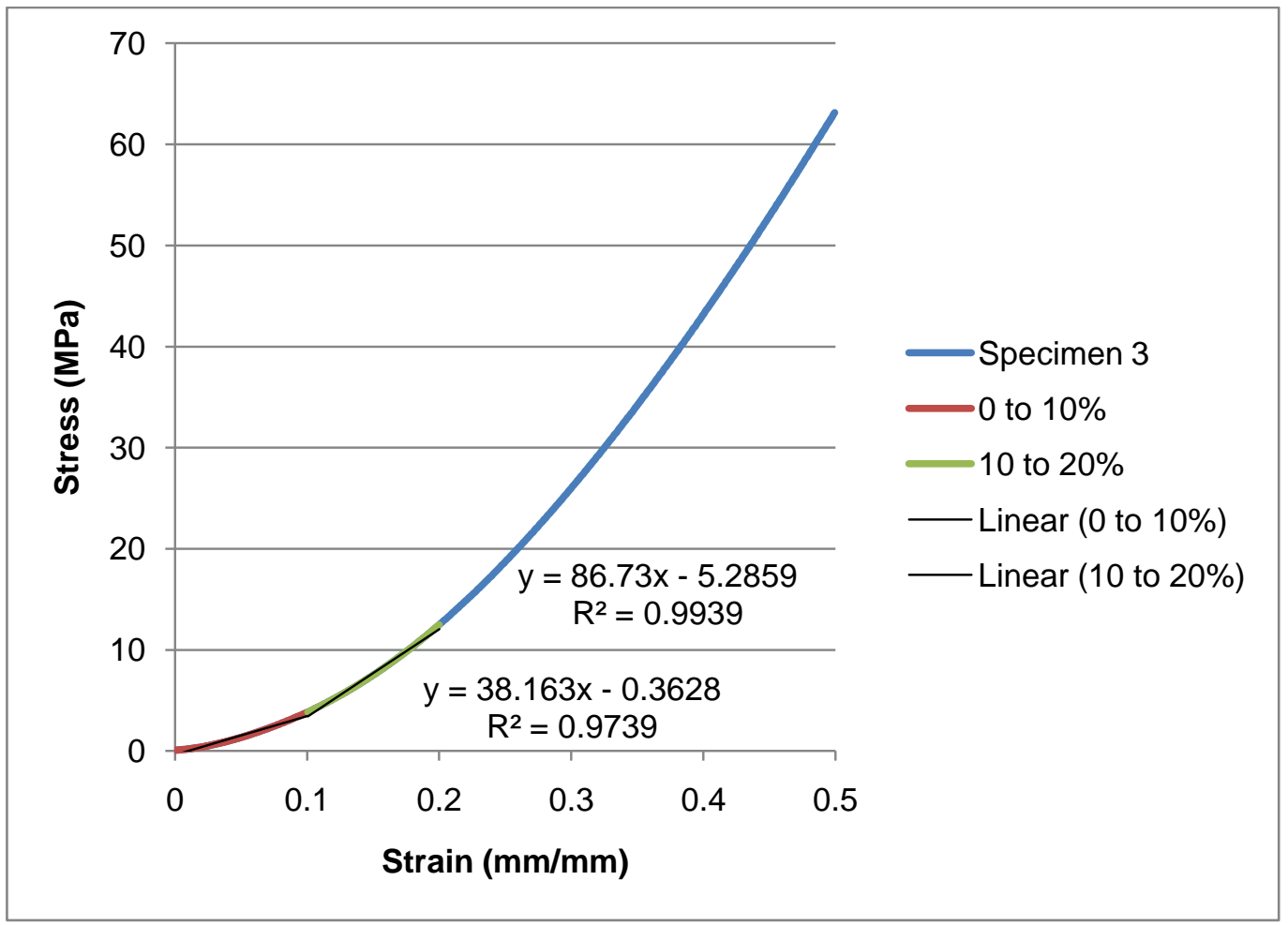

Figure B.3 Stress-Strain Behavior of dry POC-HA 80-4 Specimen 3

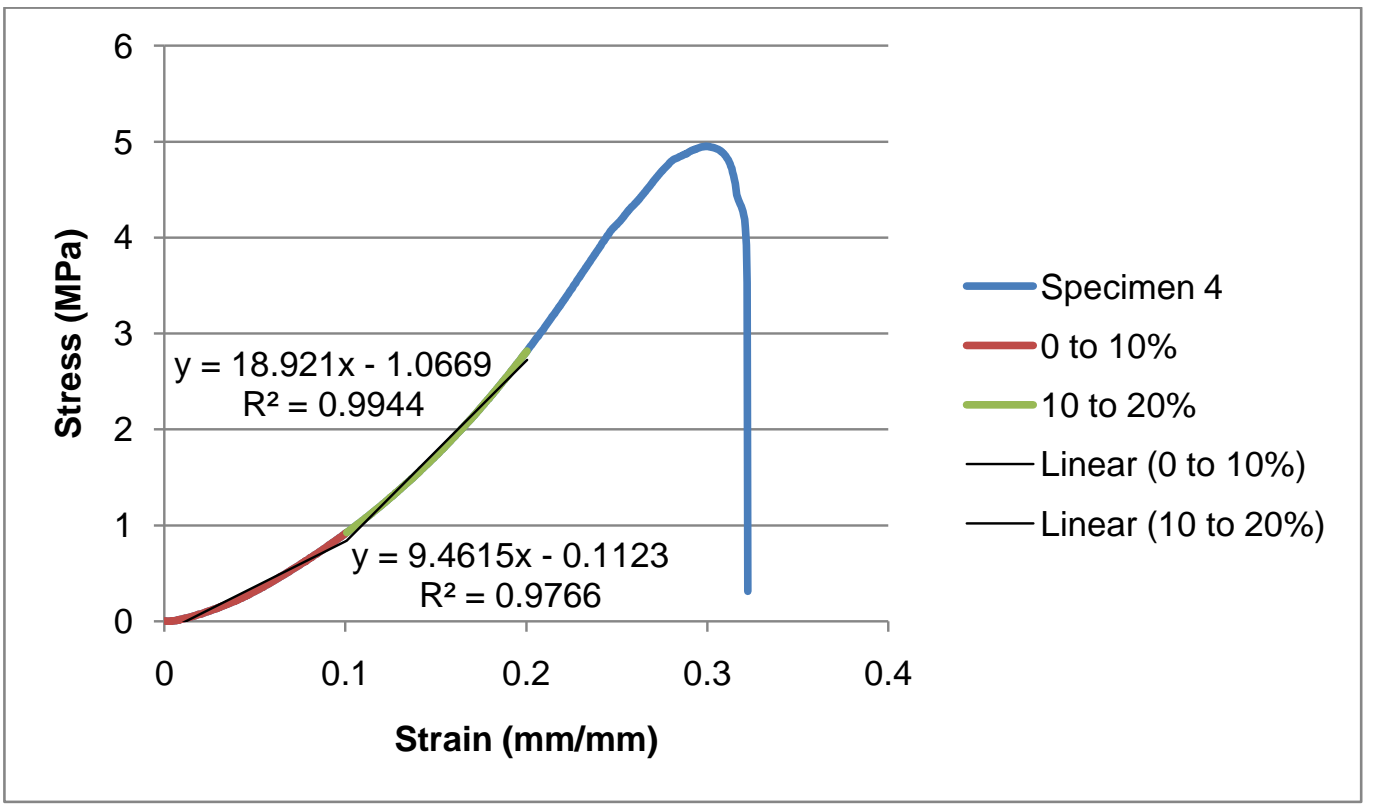

Figure B.4 Stress-Strain Behavior of wet POC-HA 80-4 Specimen 4 


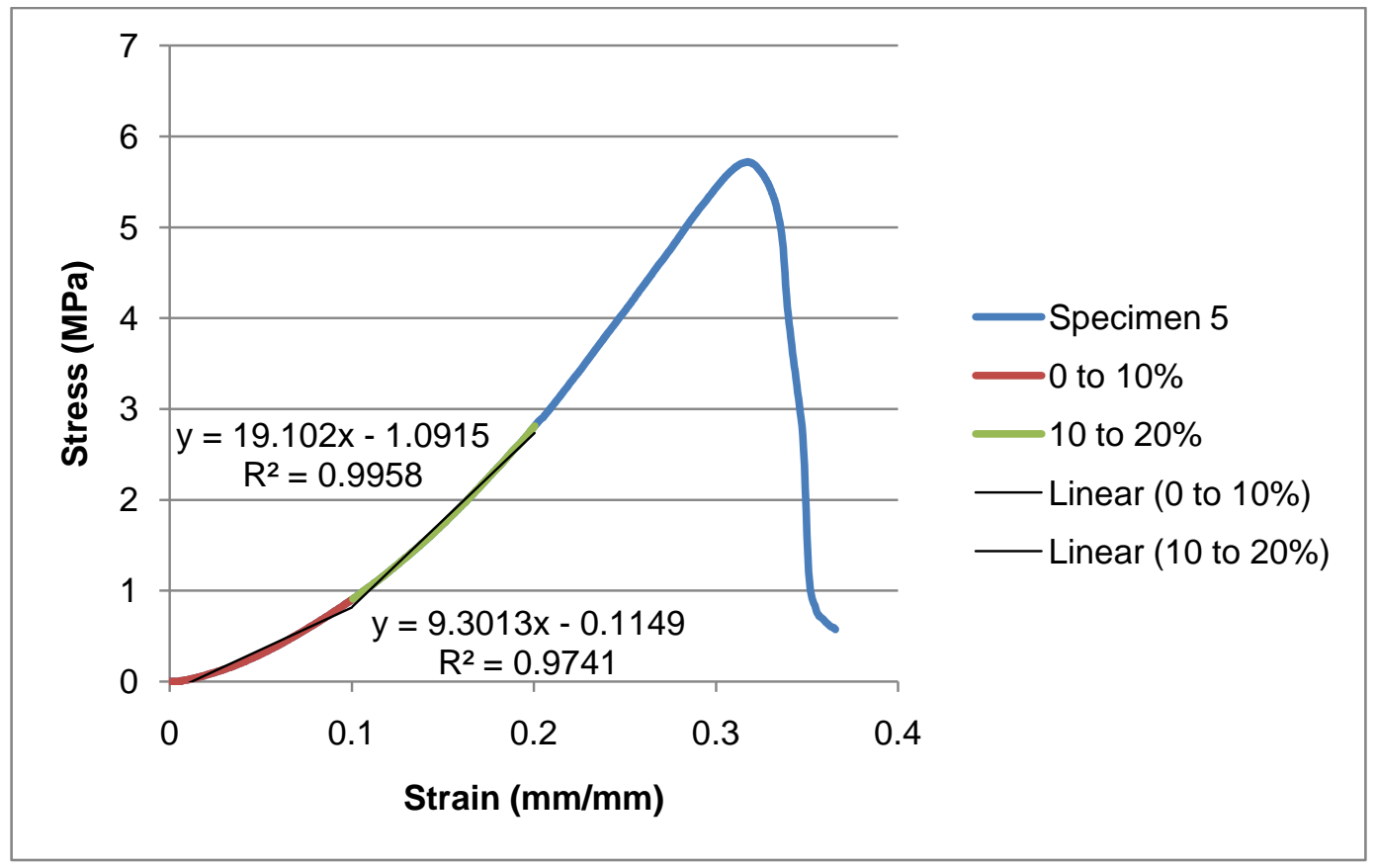

Figure B.5 Stress-Strain Behavior of wet POC-HA 80-4 Specimen 5

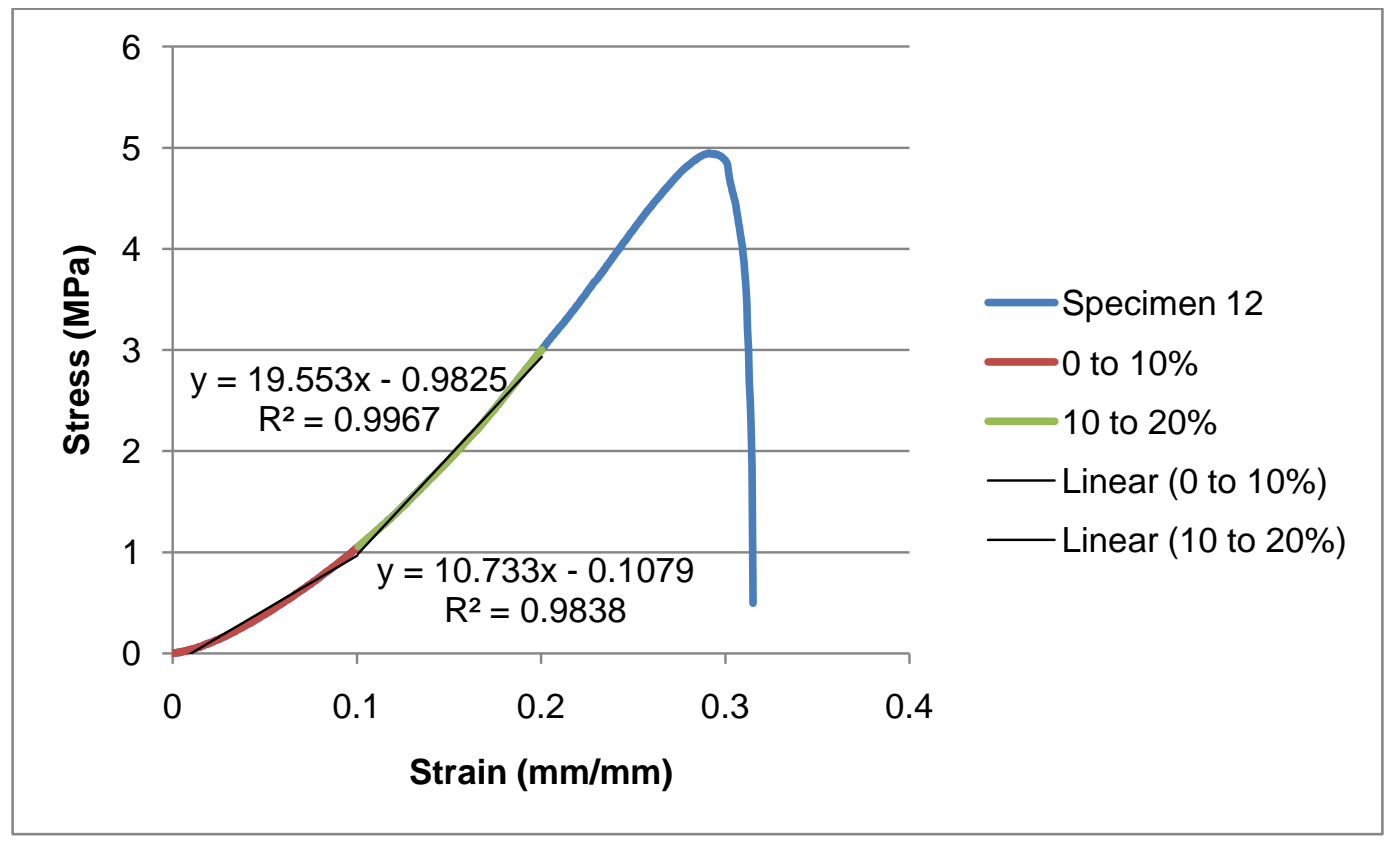

Figure B.6 Stress-Strain Behavior of wet POC-HA 80-4 Specimen 12 


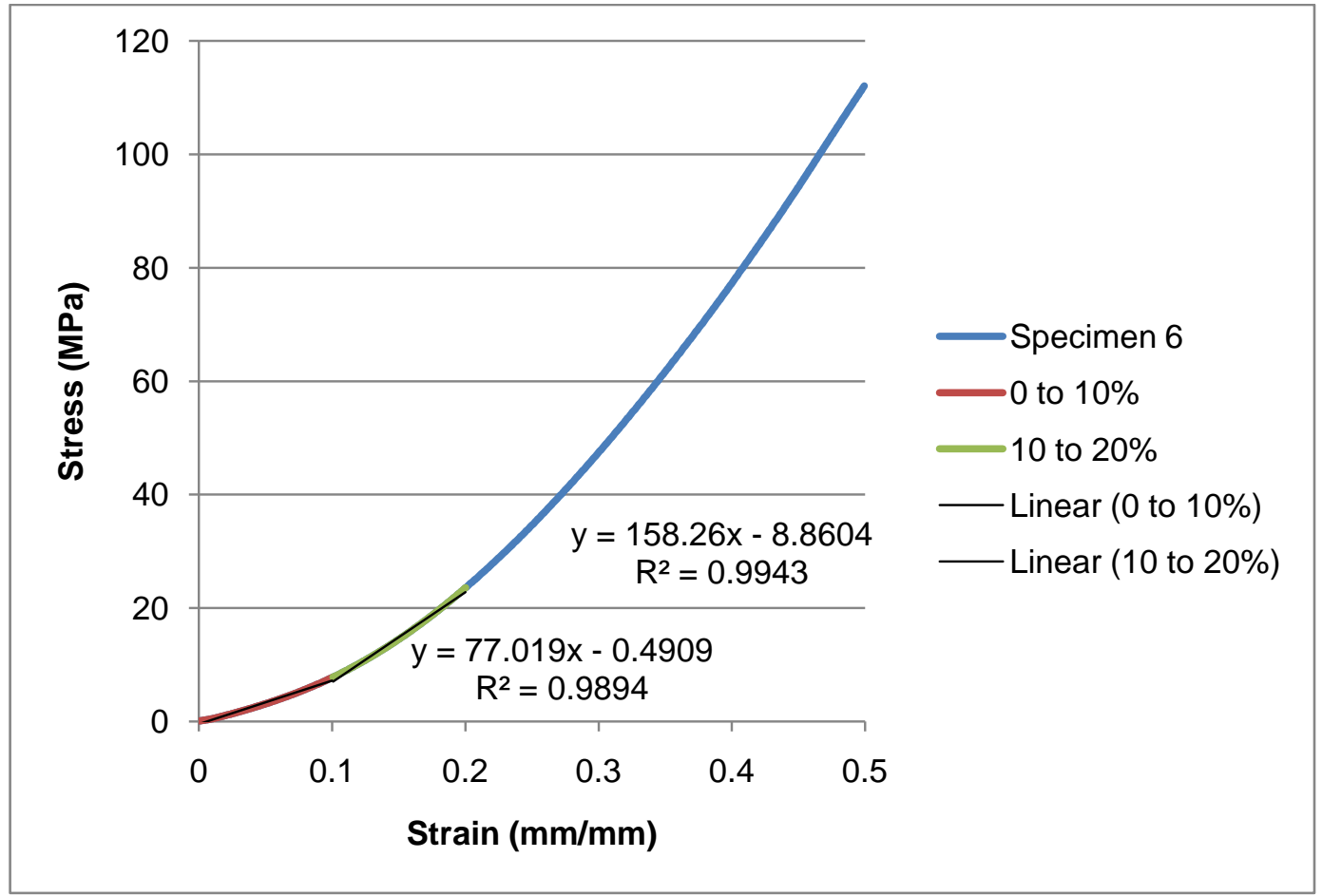

Figure B.7 Stress-Strain Behavior of dry POC-HA 120-1 Specimen 6

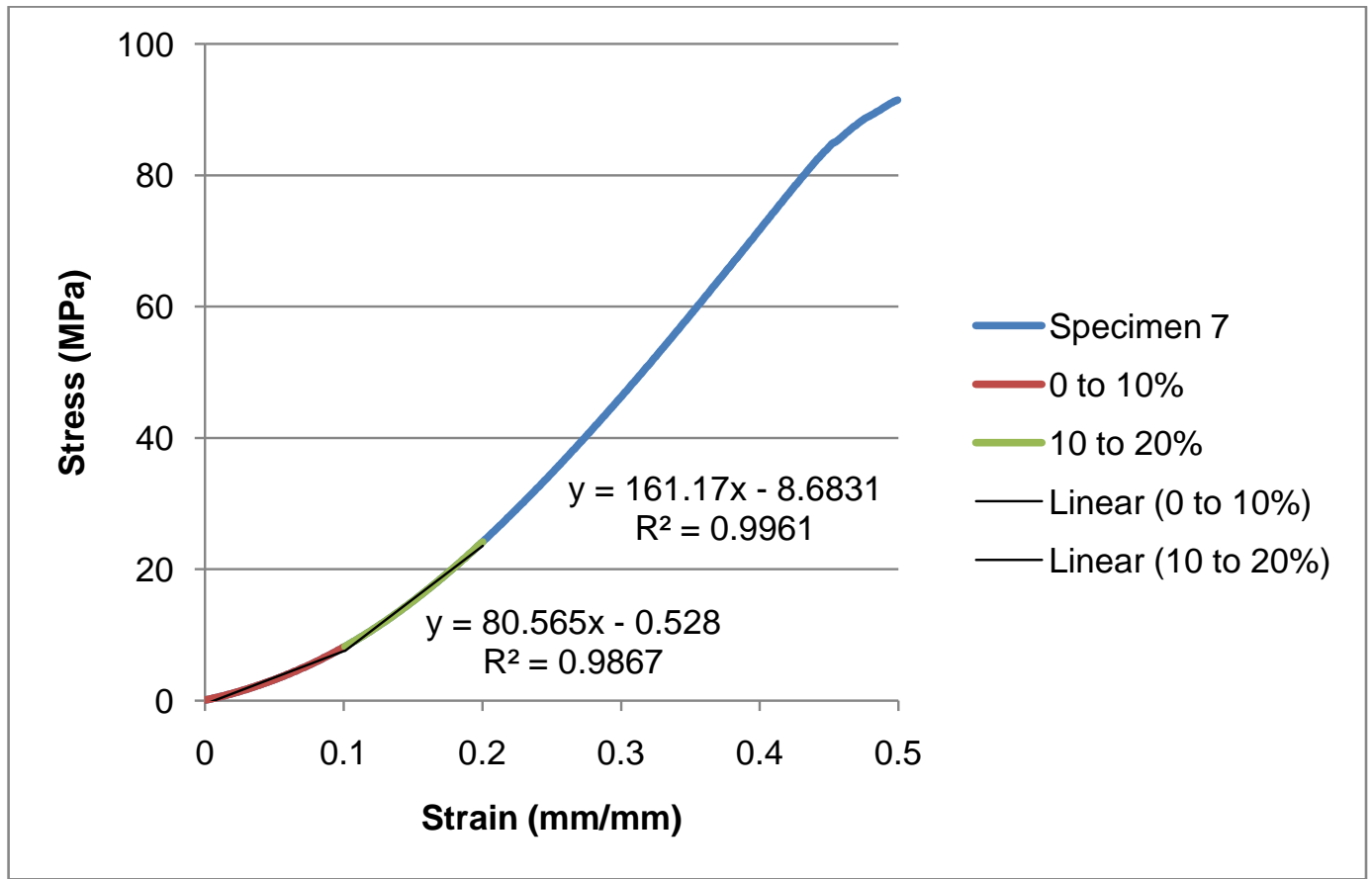

Figure B.8 Stress-Strain Behavior of dry POC-HA 120-1 Specimen 7 


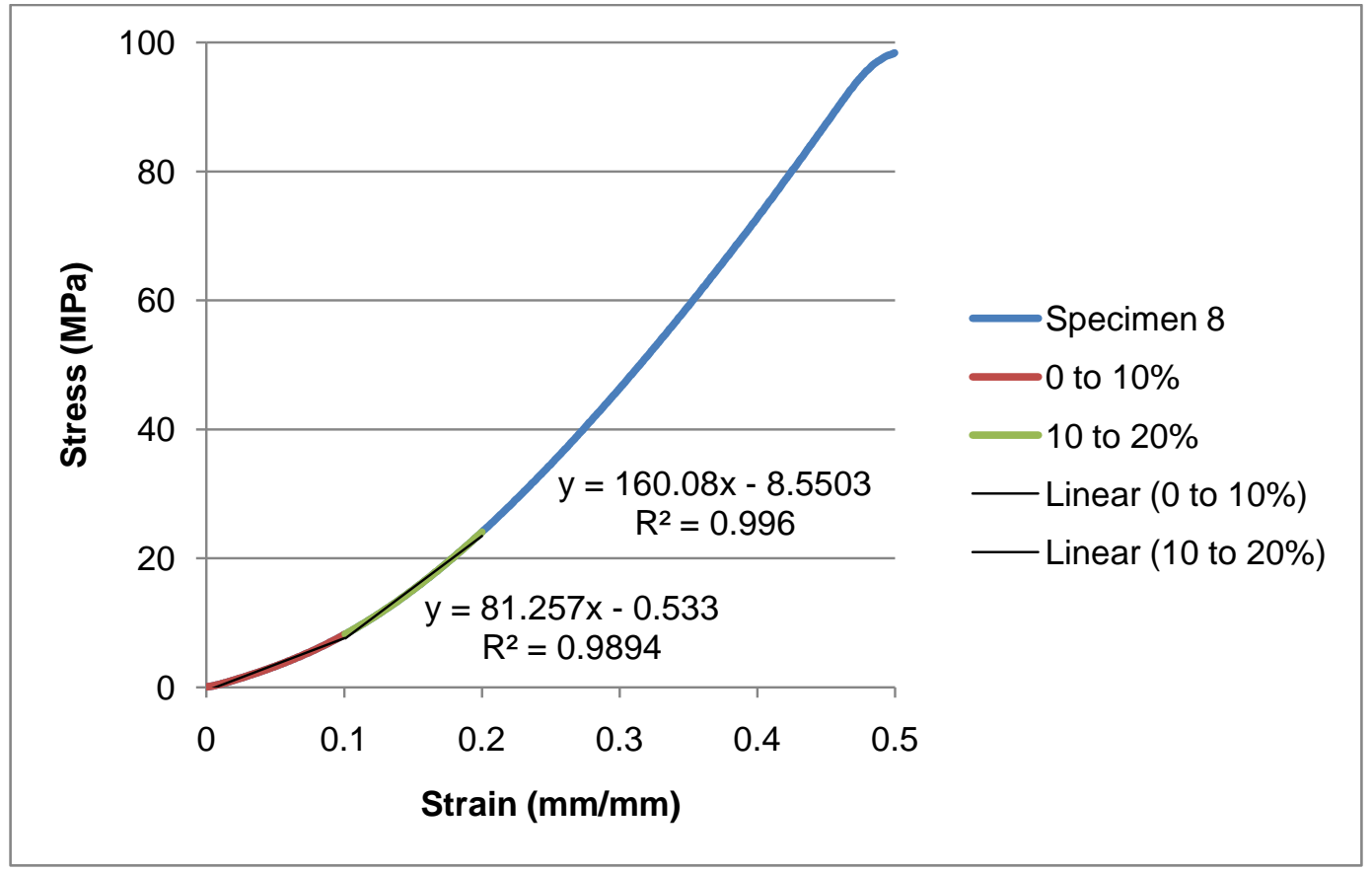

Figure B.9 Stress-Strain Behavior of dry POC-HA 120-1 Specimen 8

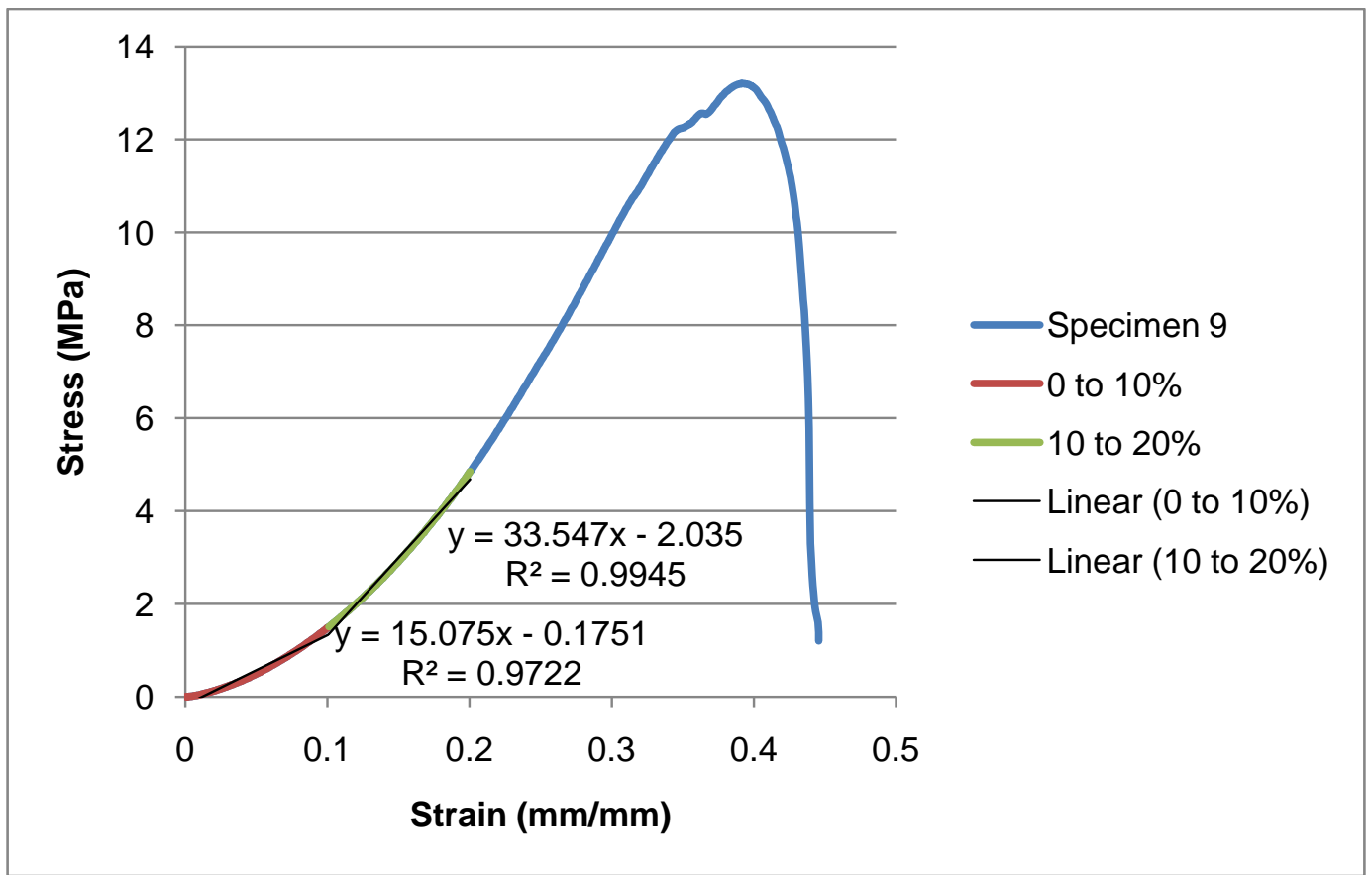

Figure B.10 Stress-Strain Behavior of wet POC-HA 120-1 Specimen 9 


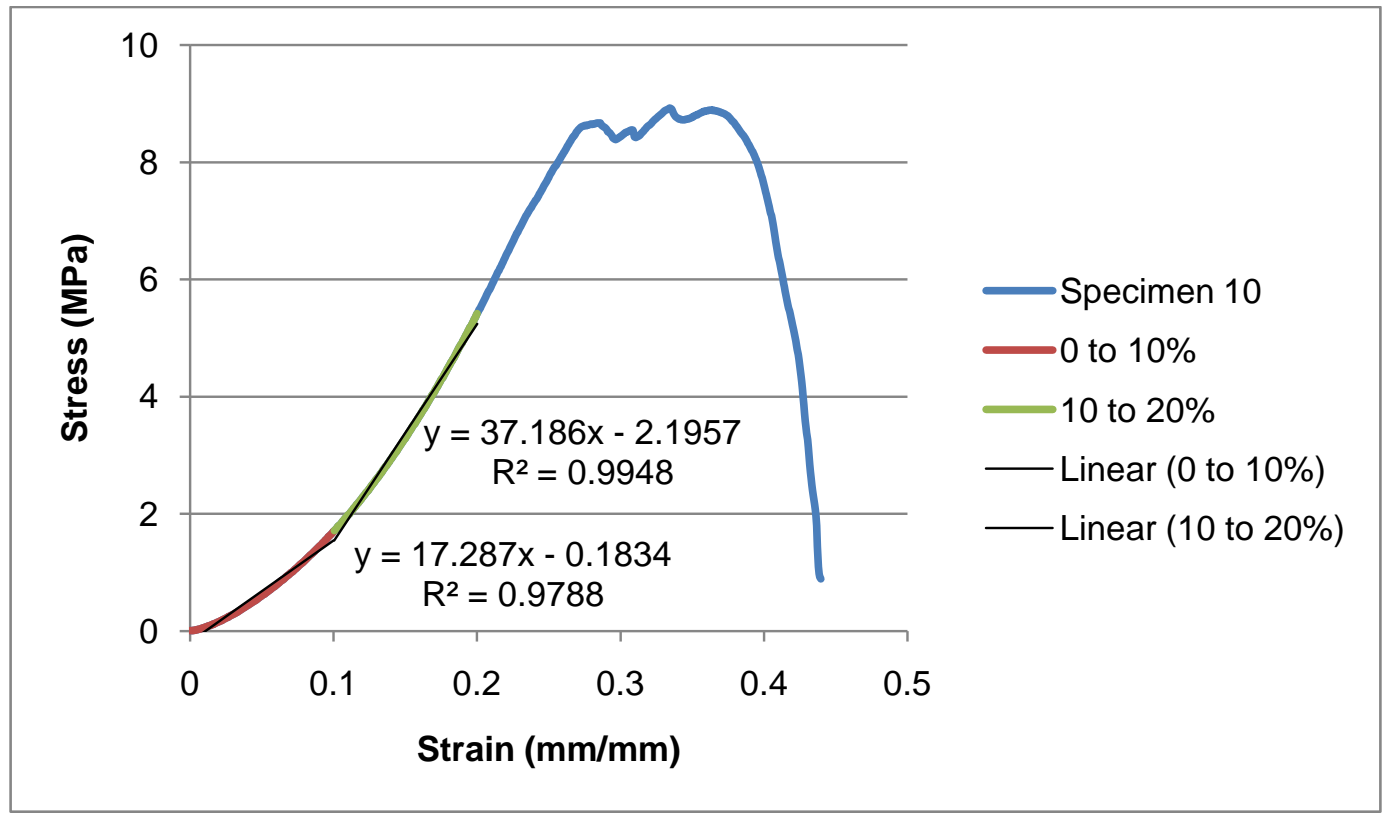

Figure B.11 Stress-Strain Behavior of wet POC-HA 120-1 Specimen 10

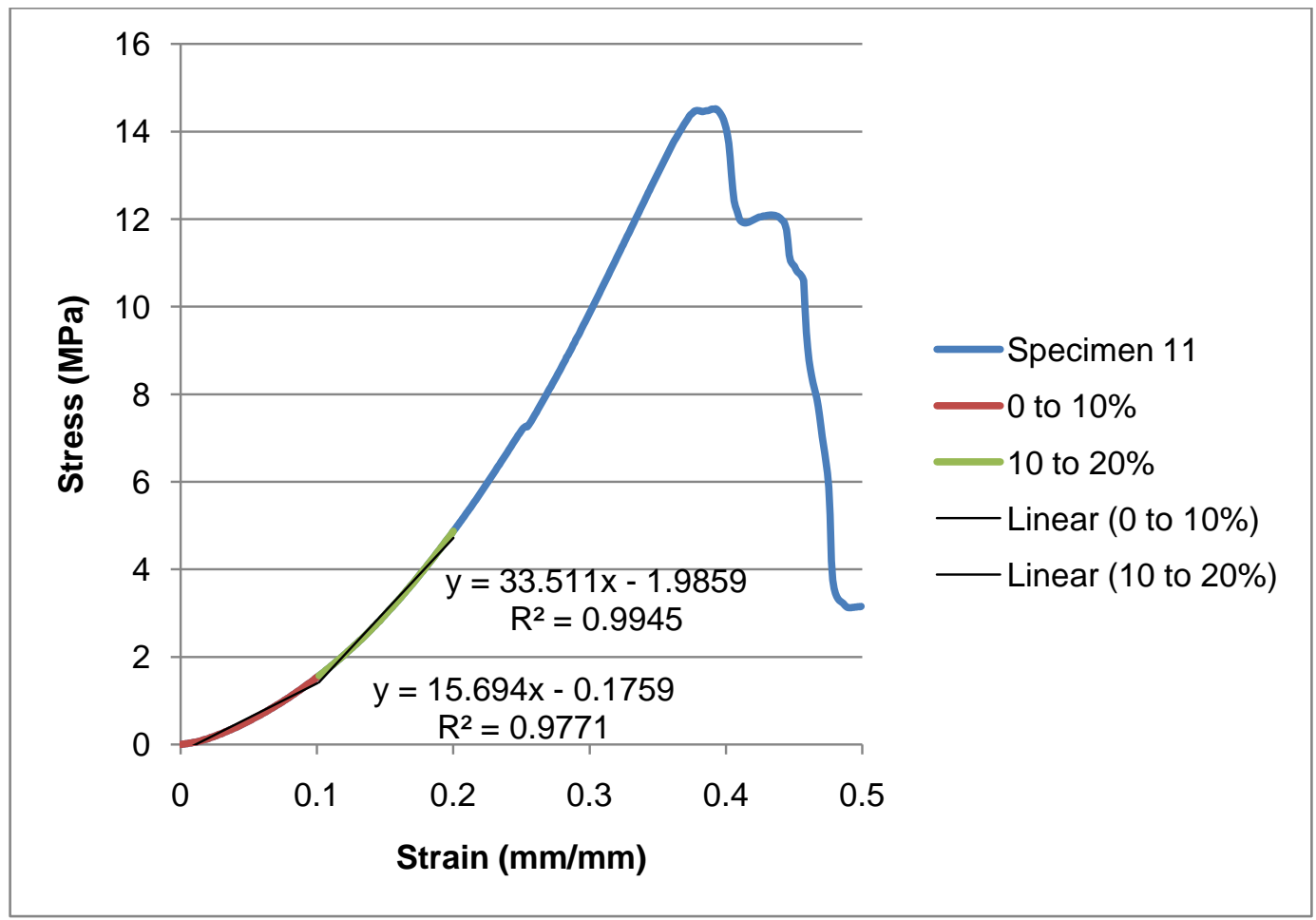

Figure B.12 Stress-Strain Behavior of wet POC-HA 120-1 Specimen 11 


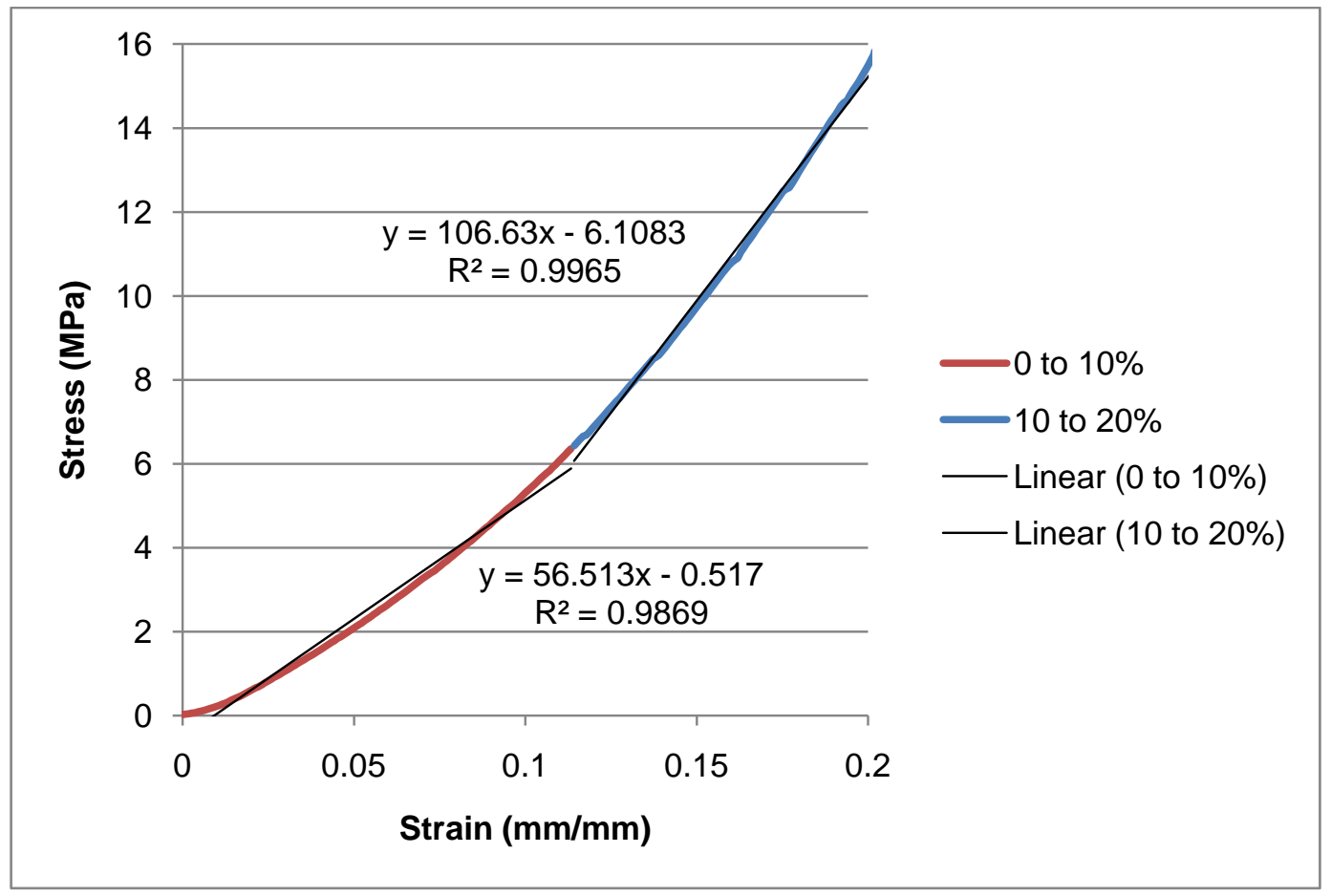

Figure B.13 Stress-Strain Behavior of dry POC-HA 80-4 Specimen 13 Test 1

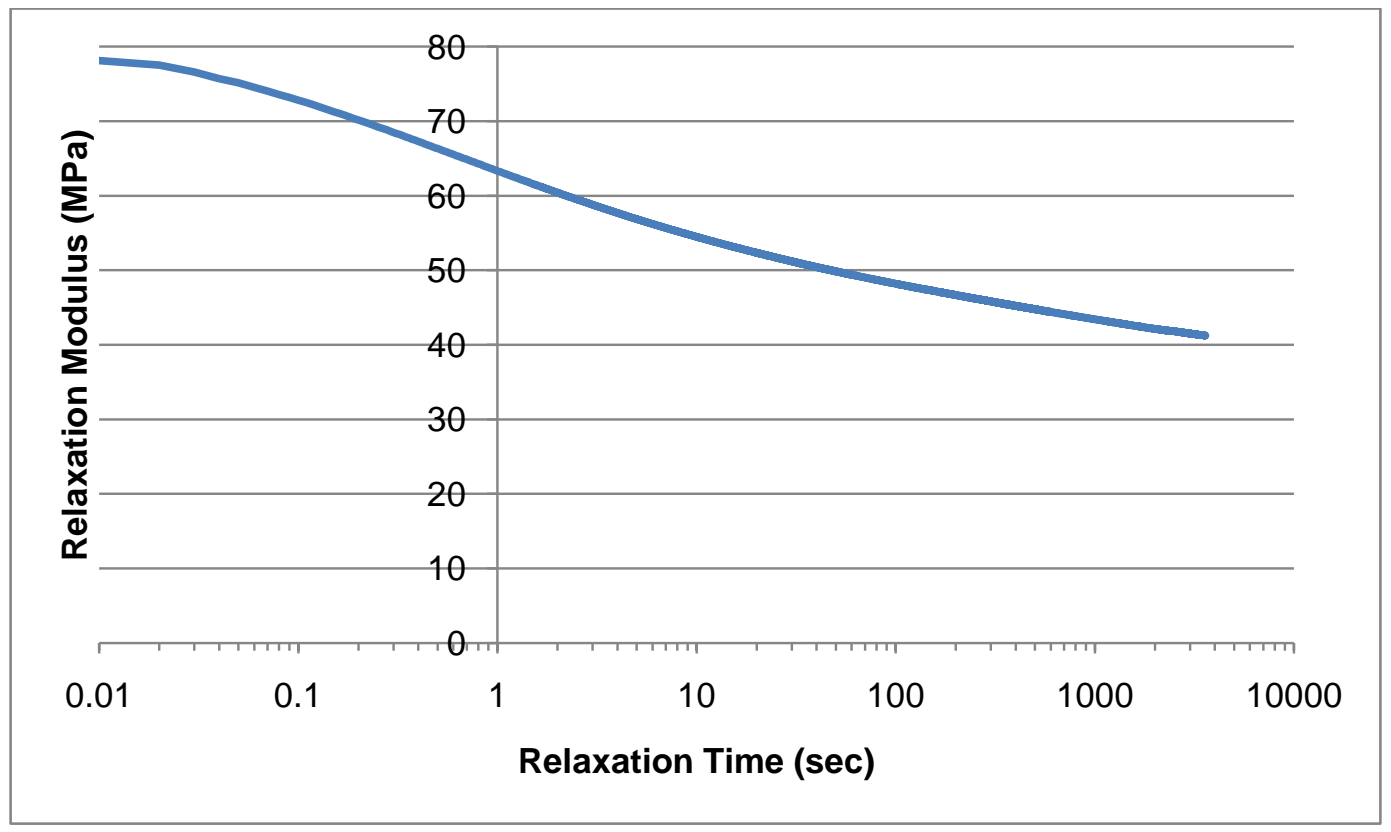

Figure B.14 Relaxation Modulus of dry POC-HA 80-4 Specimen 13 Test 1 


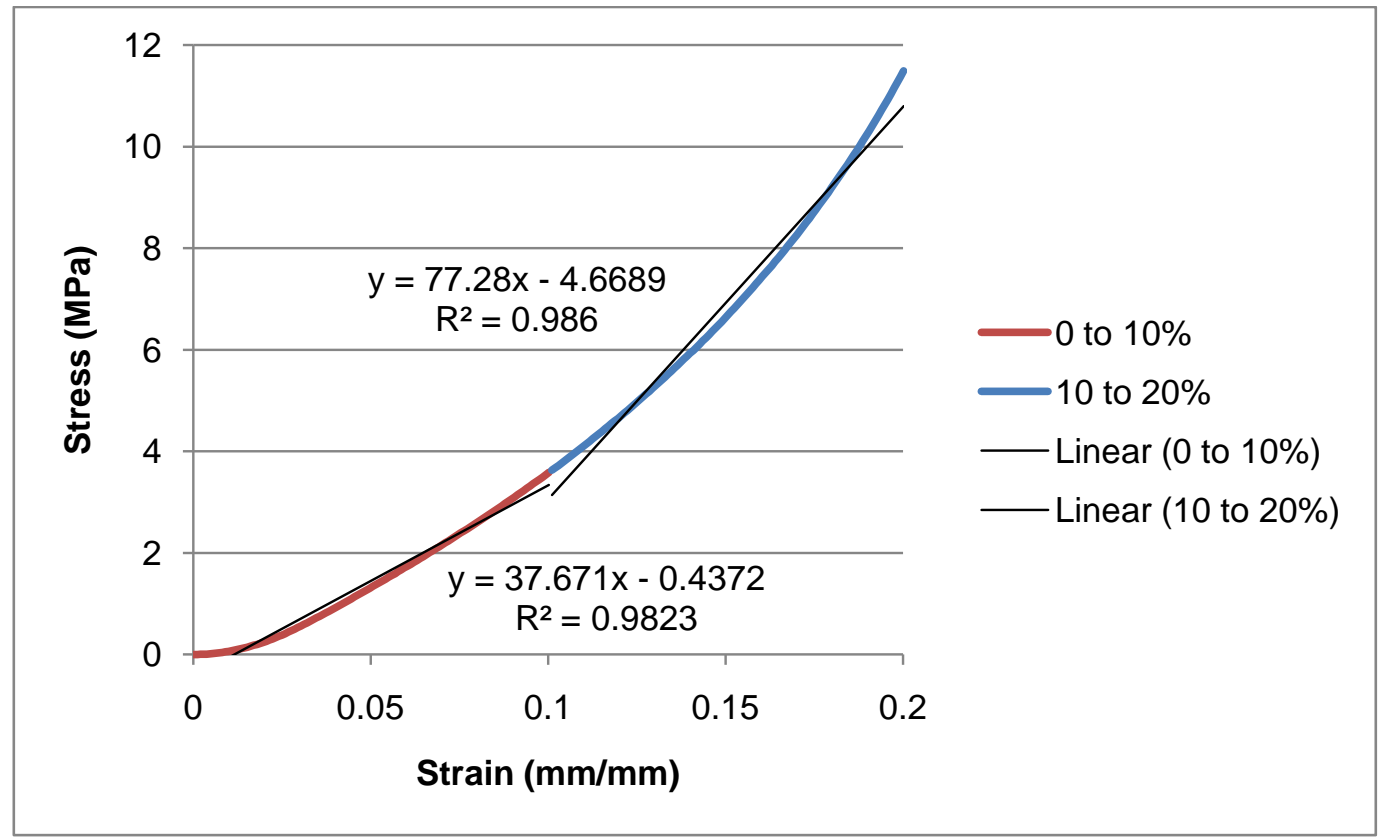

Figure B.15 Stress-Strain Behavior of dry POC-HA 80-4 Specimen 13 Test 2

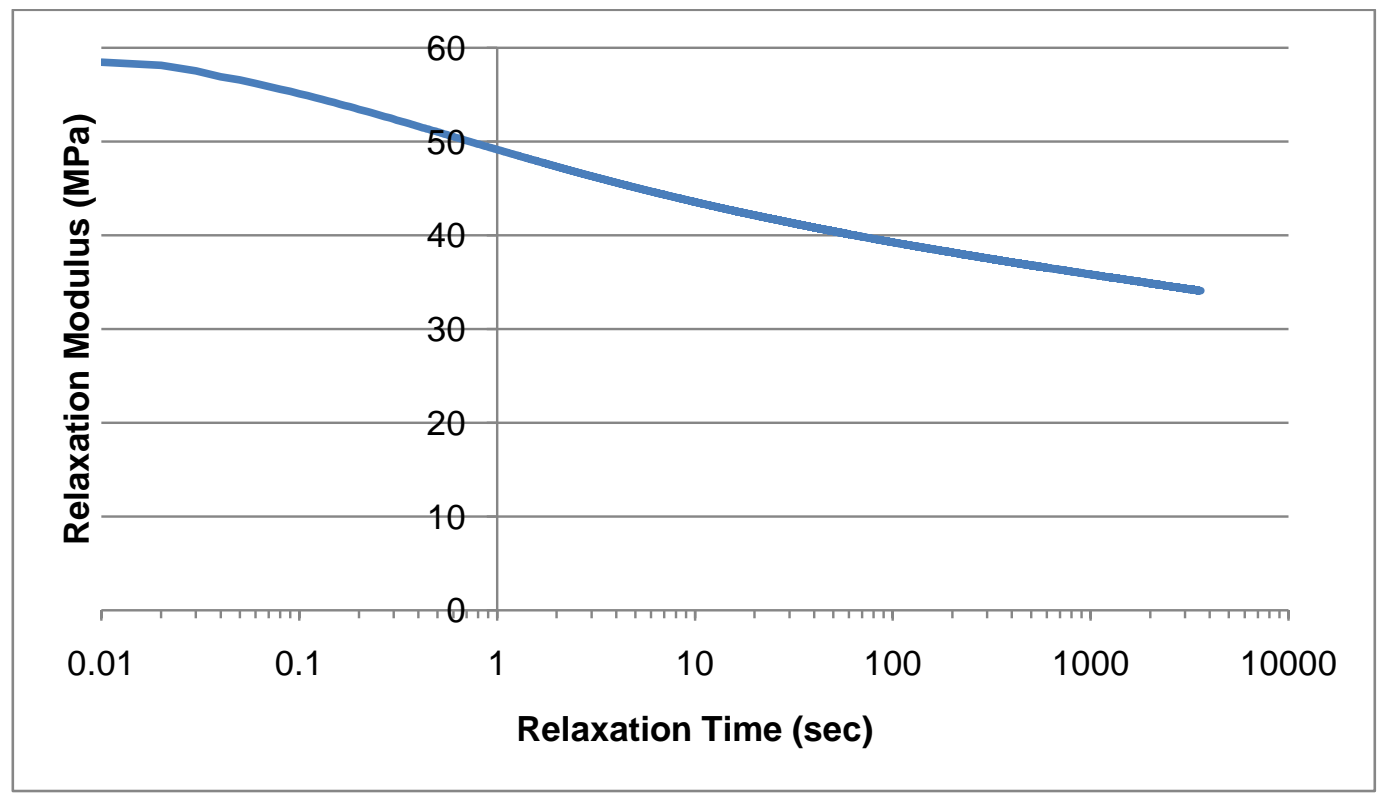

Figure B.16 Relaxation Modulus of dry POC-HA 80-4 Specimen 13 Test 2 


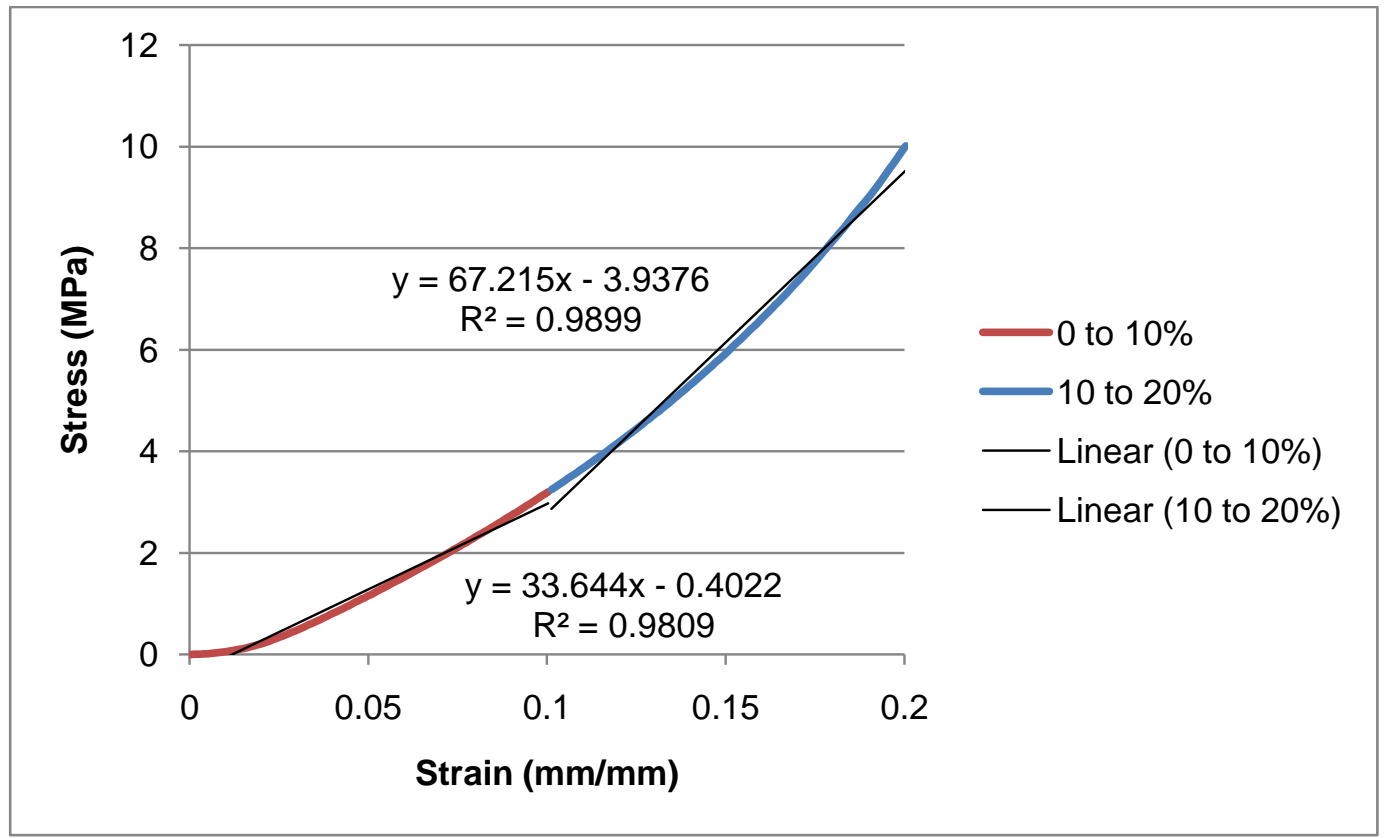

Figure B.17 Stress-Strain Behavior of dry POC-HA 80-4 Specimen 13 Test 3

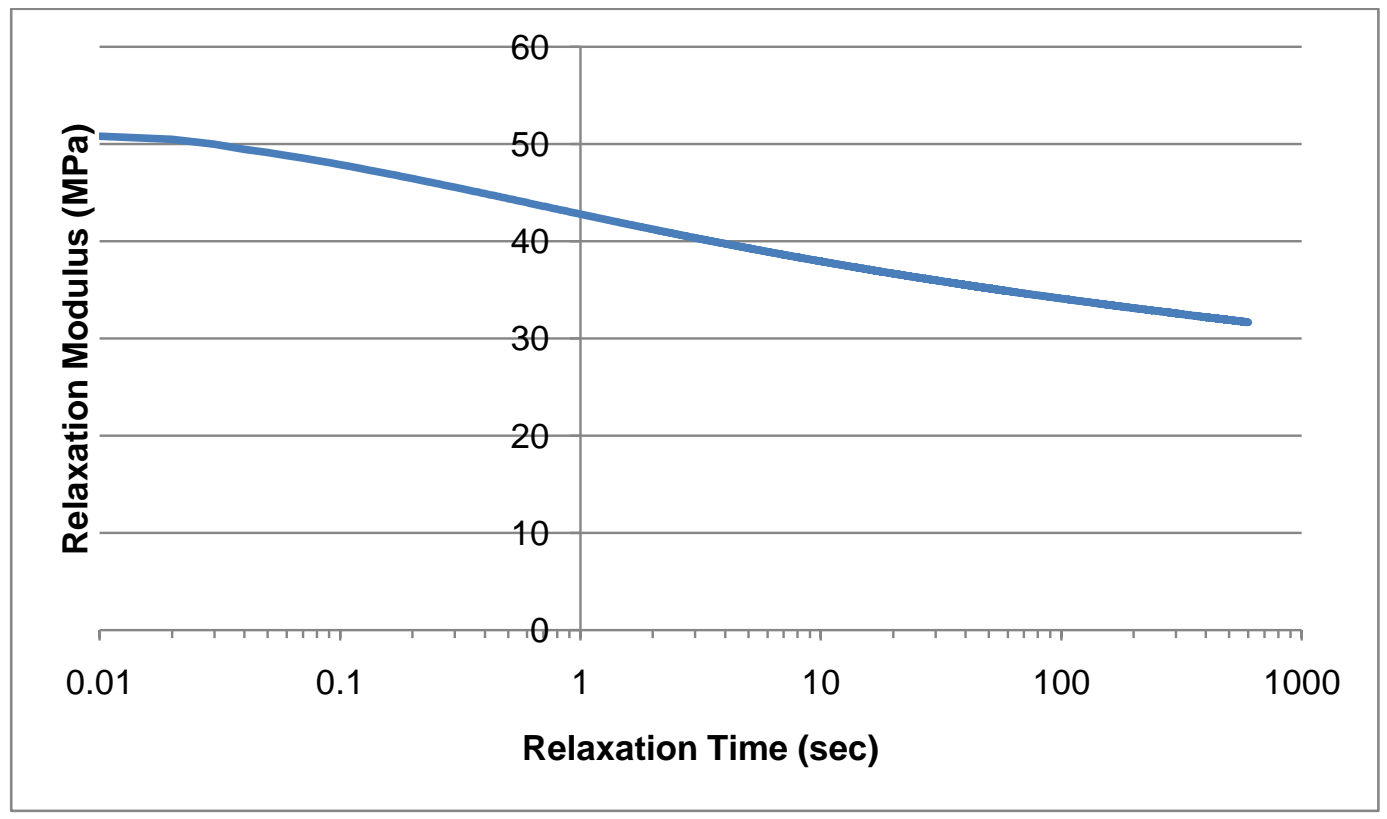

Figure B.18 Relaxation Modulus of dry POC-HA 80-4 Specimen 13 Test 3 


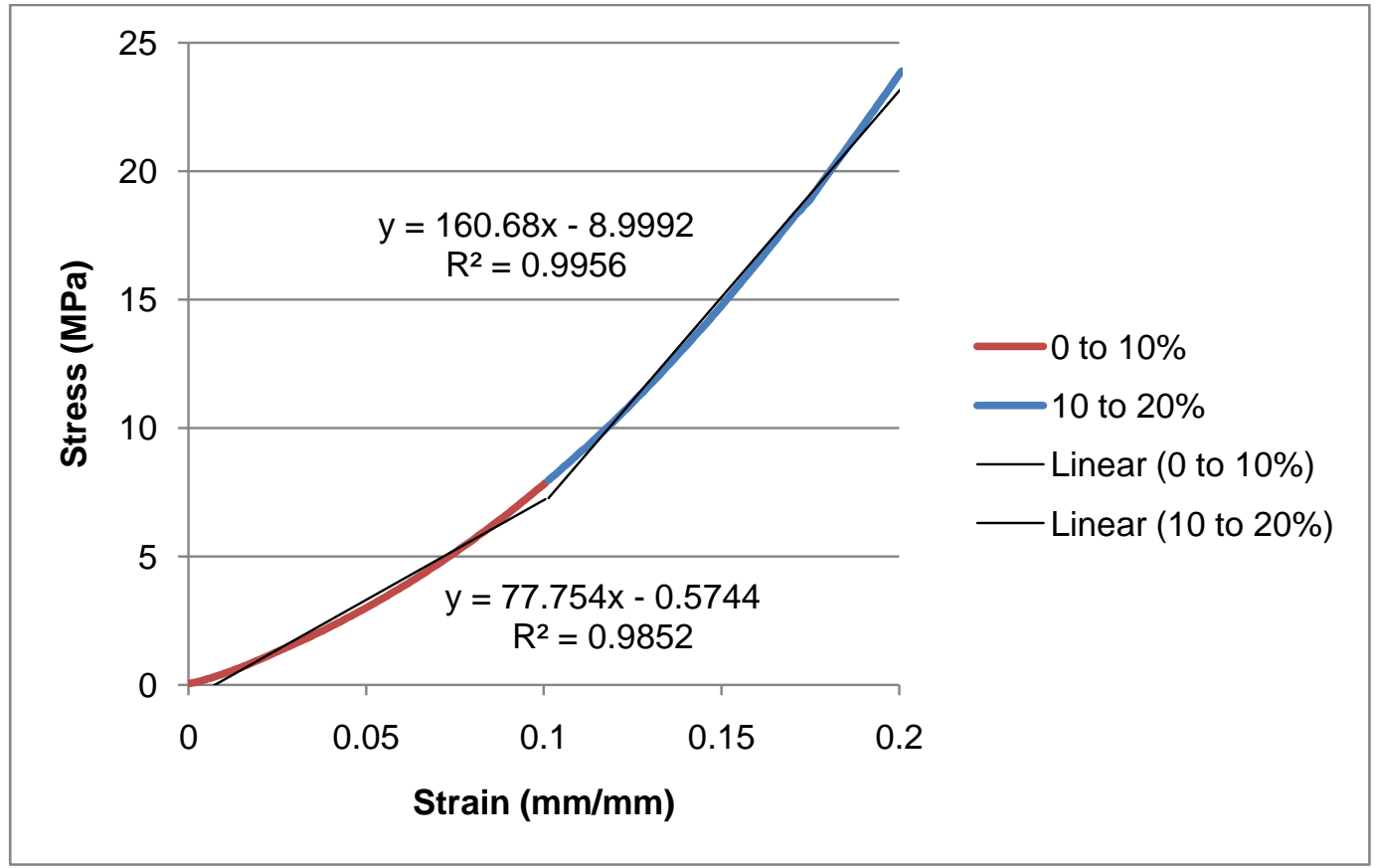

Figure B.19 Stress-Strain Behavior of dry POC-HA 120-1 Specimen 14 Test 1

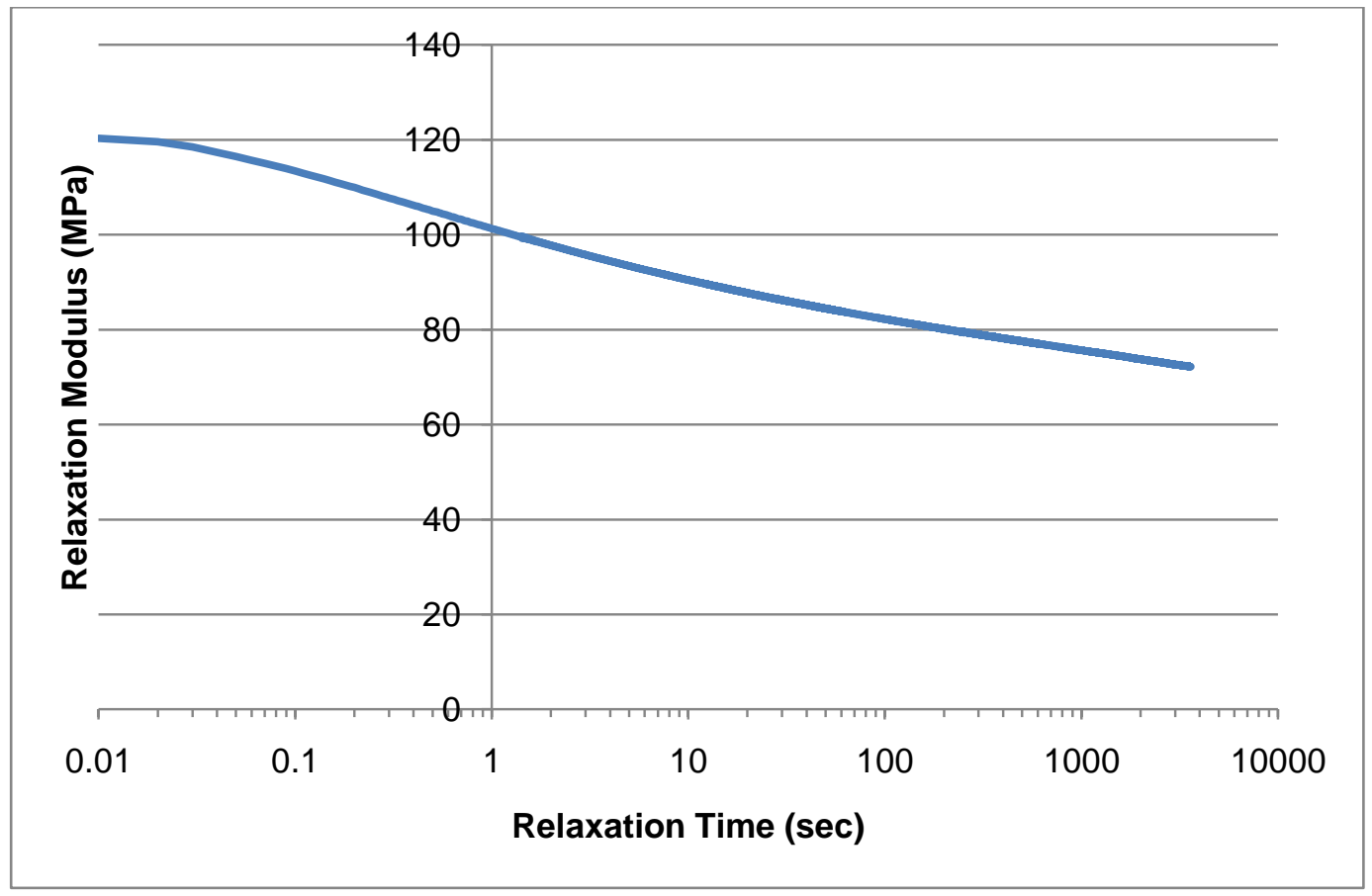

Figure B.20 Relaxation Modulus of dry POC-HA 120-1 Specimen 14 Test 1 


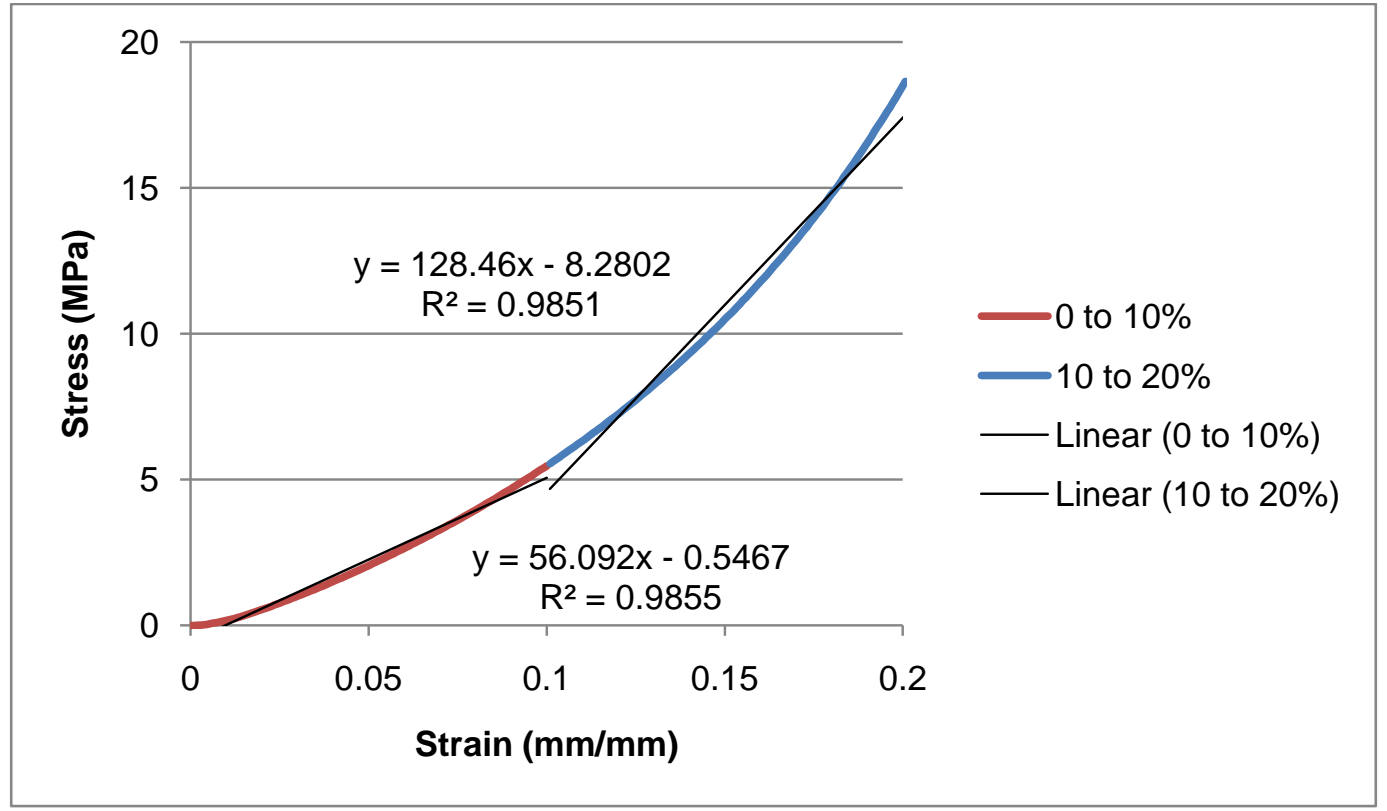

Figure B.21 Stress-Strain Behavior of dry POC-HA 120-1 Specimen 14 Test 2

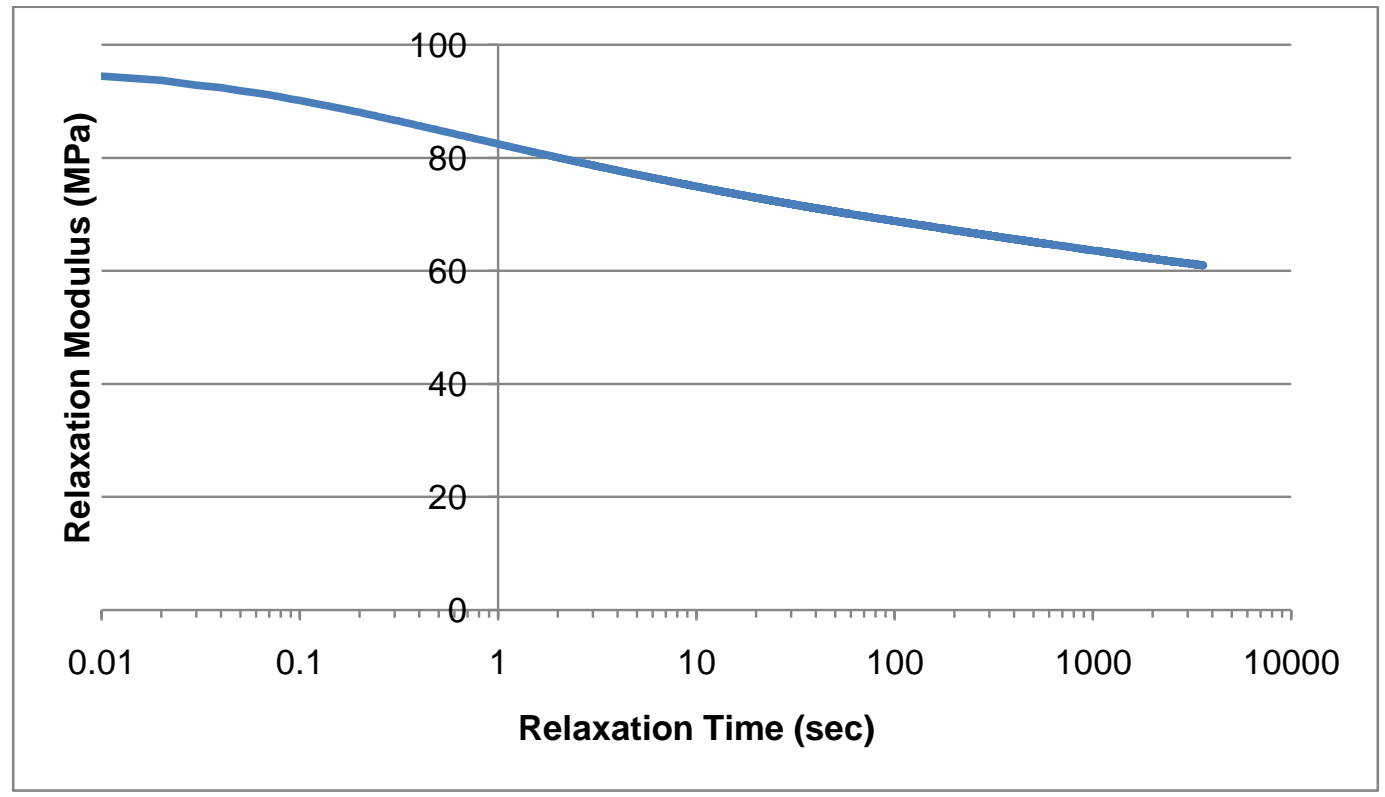

Figure B.22 Relaxation Modulus of dry POC-HA 120-1 Specimen 14 Test 2 


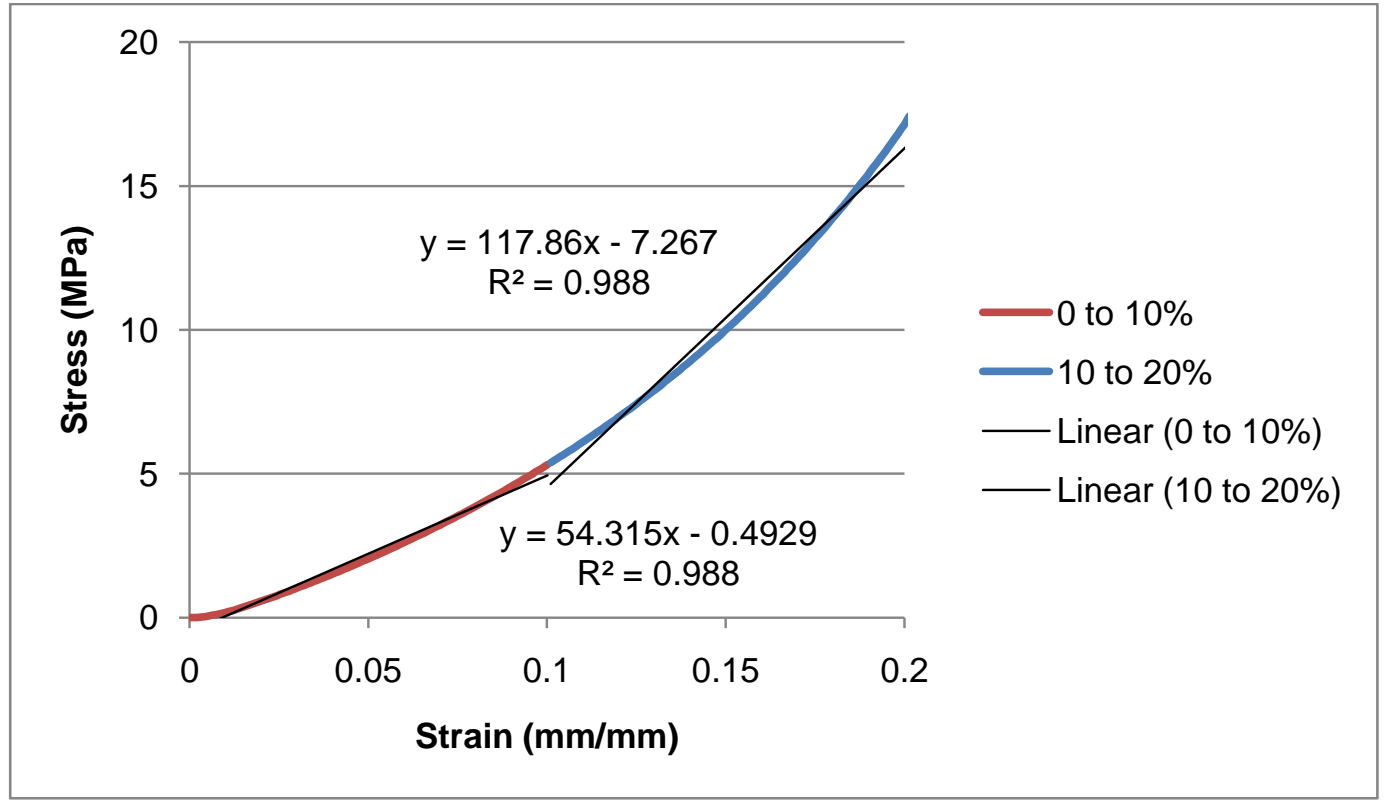

Figure B.23 Stress-Strain Behavior of dry POC-HA 120-1 Specimen 14 Test 3

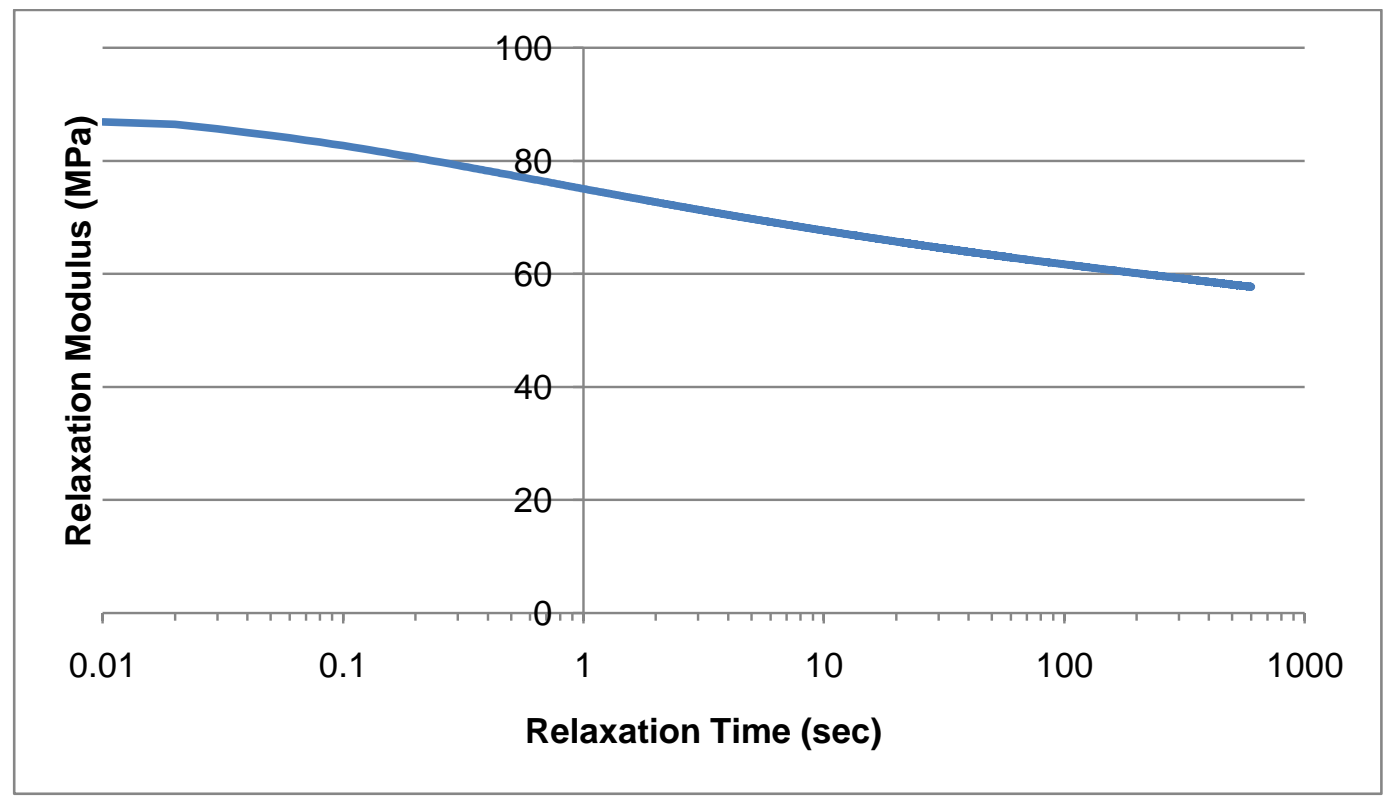

Figure B.24 Relaxation Modulus of dry POC-HA 120-1 Specimen 14 Test 3 


\section{REFERENCES}

"ANSYS Release 11.0 Documentation," ANSYS Inc., Canonsburg, PA, USA.

Brown, D., Lee, E. W., Loh, C. T., and Kee, S. T., 2009, "A New Wave in Treatment of Vascular Occlusive Disease: Biodegradable Stents-Clinical Experience and Scientific Principles," J. Vasc. Interv. Radiol., 20, pp. 315-325.

Capelli, C., Gervaso, F., Petrini, L., Dubini, G., and Migliavacca, F., 2009, “Assessment of tissue prolapse after balloon-expandable stenting: Influence of stent cell geometry," Medical Engineering \& Physics, 31, pp. 441-447.

Costa, J. R., Abizaid, A., Costa, R., Feres, F., Tanajura, L. F., Abizaid, A., Maldonado, G., Staico, R., Siqueira, D., Sousa, A., Bonan, R., and Sousa, J., 2009, "1-Year Results of the Hydroxyapatite Polymer-Free Sirolimus-Eluting Stent for the Treatment of Single De Novo Coronary Lesions," J. Am. Coll. Cardiol.: Cardiovascular Interventions, 2(5), pp. 422-427.

Grabow, N., Bunger, C., Schultze, C., Schmohl, K., Martin, D., Williams, S., Sternberg, K., and Schmitz, K.-P., 2007, "A Biodegradable Slotted Tube Stent Based on Poly(L-lactide) and Poly(4-hydroxybutyrate) for Rapid Balloon-Expansion," Annals of Biomedical Engineering, 35(12), pp. 2031-2038.

Grabow, N., Schlun M., Sternberg, K., Hakansson, N., Kramer, S., and Schmitz, K.-P., 2005, "Mechanical Properties of Laser Cut Poly(L-lactide) Micro-specimens: Implications for Stent Design, Manufacture, and Sterilization," ASME J. Biomech. Eng., 127, pp. 25-31.

Kimura T., Yokoi H., Nakagawa Y., Tamura, T., Kaburagi, S., Sawada, Y., Sato, Y., Yokoi, H., Hamasaki, N., Nosaka, H., and Nobuyoshi, M., 1996, "Three-Year Follow-up after Implantation of Metallic Coronary Artery Stents," N. Engl. J. Med., 334, pp. 561-566. 
Kukreja, N., Onuma, Y., Daemen, J., and Serruys, P., 2008, "The future of drug-eluting stents," Pharmacological Research, 57, pp.171-180.

Lanzer, P. ed., 2006, "Mastering Endovascular Techniques - A Guide to Excellence," Lippincott Williams \& Wilkins, Philadelphia, PA, pp. 114-135.

Loos, E., Rohde, R., Haverich, A., and Barlach, S., 2007, "In Vitro and In Vivo Biocompatibility Testing of Absorbable Metal Stents," Macromol. Symp. 253, pp. 103-108.

Morton, A., Crossman, D., and Gunn, J., 2004, "The influence of physical stent parameters upon restenosis," Pathologie Biologie, 52, pp. 196-205.

Nakazawa, G., Vorpahl, M., Finn, A. V., Narula, J., and Virmani, R., 2009, “One Step Forward and Two Steps Back With Drug-Eluting-Stents," J. Am. Coll. Cardiol.: Cardiovascular Imaging, 2(5), pp. 625-628.

National Heart Lung and Blood Institute, "Stents", U. S. National Institutes of Health, Bethesda, Maryland, USA, http://www.nhlbi.nih.gov/health/dci/Diseases/stents/stents all.html.

Ormiston, J. A., Serruys, P. W., Regar, E., Dudek, D., Thuesen, L., Webster, M., Onuma, Y., Garcia-Garcia, H., McGreevy, R., and Veldhof, S., 2008, "A bioabsorbable everolimus-eluting coronary stent system for patients with single de-novo coronary artery lesions (ABSORB): a prospective open-label trial," Lancet, 371, pp. 899-907.

Ozolanta, I., Tetere G., Purinya, B., and Kasyanov V., 1998, "Changes in the mechanical properties, biochemical contents and wall structure of the human coronary arteries with age and sex," Medical Engineering and Physics, 20, pp. 523-533.

Qiu, H., Yang, J., Kodali, P., Koh, J., and Ameer, G., 2006, "A Citric Acid-based Hydroxyapatite Composite for Orthopedic Implants," Biomaterials, 27, pp. 5845-5854.

Rasband, W.S., 1997-2009, "ImageJ," U. S. National Institutes of Health, Bethesda, Maryland, USA, http://rsb.info.nih.gov/ij/. 
Serruys, P. W., Ormiston, J. A., Onuma, Y., Regar, E., Gonzalo, N., Garcia-Garcia, H., Nieman, K., Bruining, N., Dorange, C., Miquel-Hébert, K., Veldhof, S., Webster, M., Thuesen, and L., Dudek, D., 2009, "A bioabsorbable everolimus-eluting coronary stent system (ABSORB): 2-year outcomes and results from multiple imaging methods," Lancet, 374, pp. 897910.

Stoeckel D, Bonsignore, C., and Duda, S., 2002, "A Survey of Stent Designs," Min. Invas. Ther. \& Allied Technol., 11(4), pp. 137-147.

Tamai, H., Igaki, K., Kyo, E., Kosuga, K., Kawashima, A., Matsui, S., Komori, H., Tsuji, T., Motohara, S., and Uehata, H., 2000, "Initial and 6-Month Results of Biodegradable Poly-ILactic Acid Coronary Stents in Humans," Circulation, 102(4), pp. 399-404.

Tanimoto, S., Bruining, N., van Domburg, R. T., Rotger, D., Radeva, P., Ligthart, J. M., and Serruys, P. W., 2008, "Late Stent Recoil of the Bioabsorbable Everolimus-Eluting Coronary Stent and its Relationship With Plaque Morphology," J. Am. Coll. Cardiol.: Interventional Cardiology, 52(20), pp. 1616-1620.

Uppal, N., 2005, "Femtosecond Laser Micromachining of Engineering Materials: Process Parameters Study and Microrapid Prototyping," M.S. Thesis, The University of Texas at Arlington.

Venkatraman, S., Boey, F., and Lao, L. L., 2008, "Implanted cardiovascular polymers: Natural, synthetic and bio-inspired," Progress in Polymer Science, 33, pp. 853-874.

Welch, T., Eberhart, R. C., and Chuong, C.-J., 2008, "Characterizing the Expansive Deformation of a Bioresorbable Polymer Fiber Stent," Annals of Biomedical Engineering, 36(5), pp. $742-751$.

Wu, L., Zhang, J., Jing, D., and Ding, J., 2006, “"Wet-state” mechanical properties of three-dimensional polyester porous scaffolds," J. Biomed. Mater. Res., 76A, pp. 264-271. 


\section{BIOGRAPHICAL INFORMATION}

Joonas Ponkala received his Honors Bachelor of Science in Mechanical Engineering from The University of Texas at Arlington in December 2006. He held a graduate engineer position at Meadows Analysis \& Design, LLC where he performed finite element simulations and stress analysis to verify mechanical designs. He returned to UTA in January 2008 to further his education and knowledge in Mechanical Engineering. He has interned at Nokia Inc. where he performed dynamic finite element analyses on structures of mobile phones. His research interests are in finite element analysis, structural analysis, and biomedical applications. He plans to pursue a doctoral degree in Mechanical Engineering in the future. 